\title{
Resonance frequency tuning in vibration-based energy harvesting systems
}

Madinei, Hadi

How to cite:

Madinei, Hadi (2018) Resonance frequency tuning in vibration-based energy harvesting systems. Doctoral thesis, Swansea University.

http://cronfa.swan.ac.uk/Record/cronfa51431

Use policy:

This item is brought to you by Swansea University. Any person downloading material is agreeing to abide by the terms of the repository licence: copies of full text items may be used or reproduced in any format or medium, without prior permission for personal research or study, educational or non-commercial purposes only. The copyright for any work remains with the original author unless otherwise specified. The full-text must not be sold in any format or medium without the formal permission of the copyright holder. Permission for multiple reproductions should be obtained from the original author.

Authors are personally responsible for adhering to copyright and publisher restrictions when uploading content to the repository.

Please link to the metadata record in the Swansea University repository, Cronfa (link given in the citation reference above.)

http://www.swansea.ac.uk/library/researchsupport/ris-support/ 


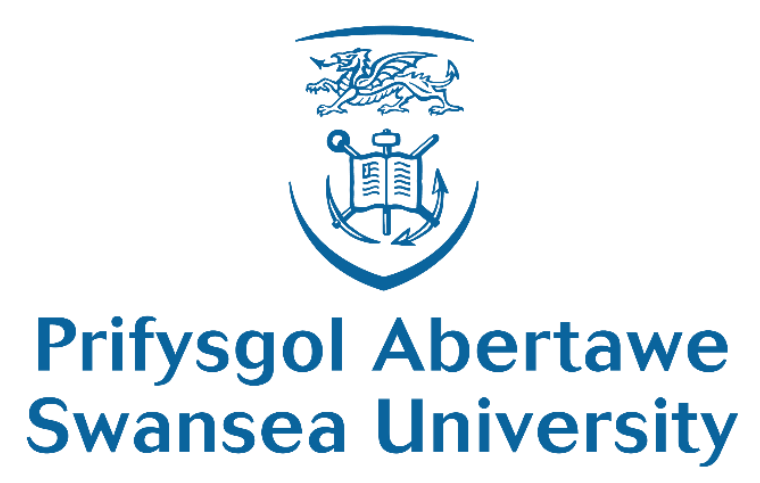

\title{
Resonance frequency tuning in vibration-based energy harvesting
} systems

\author{
Hadi Madinei
}

Supervised by

Dr. Hamed Haddad-Khodaparast

Prof. Sondipon Adhikari

Submitted to Swansea University in fulfilment of the requirements for the degree of

Doctor of Philosophy in

Mechanical Engineering

Swansea University

May 2018 
To my wife, Sahar 


\section{Abstract}

Energy harvesting technologies that rely on the conversion of ambient vibration into a usable form of energy have become the subject of significant research in recent years . The most common types of transduction methods are piezoelectric, electromagnetic and electrostatic. Among these three methods, piezoelectric convertors have been recognized to offer more benefits. They have presented a potential solution to the problem of power systems which have a short battery life and high maintenance costs. Battery replacement is more of a problem for Micro Electro Mechanical Systems (MEMS). For some applications, often it is not practical to replace the dead battery because they are not easily accessible. Therefore, the concept of low-power MEMS devices that are able to scavenge, or harvest energy from their operating environment have gained growing attention over recent years. In this thesis, an overview of energy harvesting technology based on different transduction methods is presented and discussed in detail.

Most energy harvesters are designed to work at resonance frequency in order to obtain maximum output power, and they are usually manufactured to have resonance frequencies that match the frequencies of excitation. However, in some cases, there is a mismatch between the resonance and excitation frequencies due to manufacturing errors or changes in the working environment. Particularly, in MEMS devices due to the fabrication process such as mask alignment, deposition, photolithography, etching and drying, manufacturing tolerances are generally high and, in some cases, can be higher than $\pm 10 \%$ of nominal values. Therefore, parameter uncertainty can significantly affect the performance of MEMS devices. To overcome this problem, a MEMS piezoelectric harvester with electrostatically adjustable resonance frequency is proposed. The main aim is to control the resonance frequency of the piezoelectric harvester with the application of a DC voltage to the electrostatic system in order to maximize the harvested power.

Based on the voltage applied to the electrostatic system, the resonance frequency of the harvester can be adjusted through hardening and softening mechanisms. The problem addressed in this thesis is non-linear due to electrostatic forces. Moreover, by considering uncertainty in the model parameters; we are dealing with a dynamic problem with the effects of both nonlinearities and uncertainties which has not received significant attention in the literature. In this study, for the first time to our knowledge, the shooting method in conjunction with Monte Carlo Simulation has been used to solve a nonlinear uncertain problem. In addition, due to the similarity between electrostatic and electromagnetic forces, an experimental set-up based on the nonlinear electromagnetic forces has been designed to show the concept of the proposed model in macro scales. The experimental results have been verified numerically and it has been shown that the proposed model has great potential in practical applications. 


\section{Declarations and statements}

1. This work has not previously been accepted in substance for any degree is not being concurrently submitted in candidature for any degree.

Signed.

(candidate)

Date.

2. This thesis is the result of my own investigations, except where otherwise stated. Other sources are acknowledged by footnotes giving explicit references. A bibliography is appended.

Signed

(candidate)

Signed

(supervisor)

Date.

3. I hereby give consent for my thesis, if accepted, to be available for photocopying and for inter-library loan, and for the title and summary to be made available to outside organizations.

Signed

(candidate)

Date. 


\section{Table of Contents}

\section{Contents}

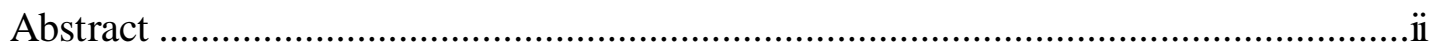

Table of Contents ..........................................................................................

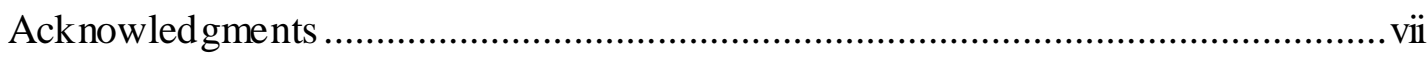

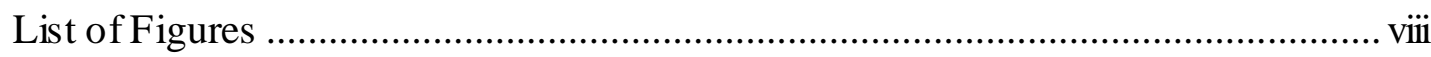

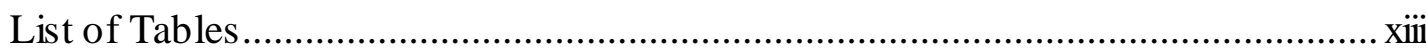

List of Symbols and Abbreviations ..................................................................

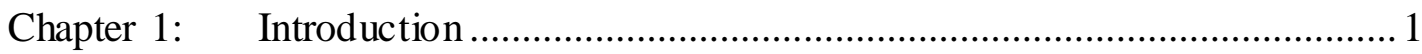

1.1. Microelectromechanical systems (MEMS) ….......................................... 1

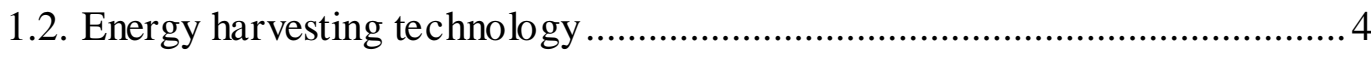

1.2.1. Piezoelectric energy harvesters ................................................ 5

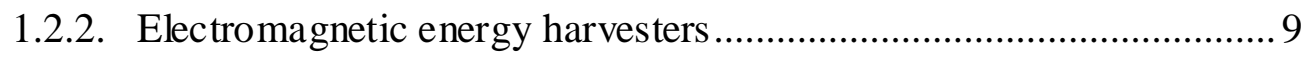

1.2.3. Electrostatic energy harvesters …................................................ 10

1.3. Review of MEMS vibration-based energy harvesters................................ 14

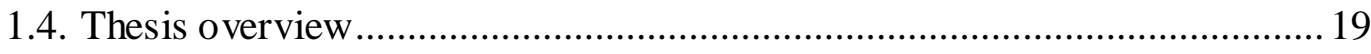

1.5. Journal and conference papers based on this the sis ..................................2 20

Chapter 2: $\quad$ Modelling of cantilevered piezoelectric energy harvesters ............... 23

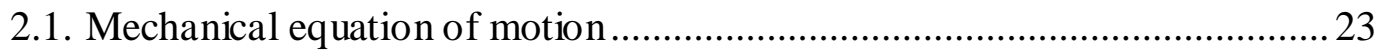

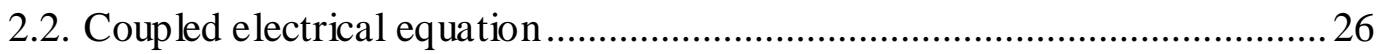

2.2.1. Coupled electrical equation for series and parallel connections .......28 
2.4. Theoretical case study

2.5. Closure 39

Chapter 3: Modelling and design of MEMS piezoelectric energy harvester with adjustable resonance frequency 40

3.1. Model description and mathematical modelling 40

3.2. Numerical Results and Discussion 47

3.2.1. Parametric study 47

3.2.2. Sustainability of the proposed harvester 54

3.2.3. Design strategy 56

3.3. Closure 61

Chapter 4: Effects of manufacturing uncertainties in MEMS Energy Harvesters. .62

4.1. Introduction to Uncertainty Analysis 62

4.2. Representation and Propagation of Uncertainty 63

4.2.1. Representation 63

4.2.2. Uncertainty Propagation 64

4.3. Manufacturing uncertainties in MEMS devices 65

4.4. Model description and mathematical modelling .66

4.5. Numerical results and discussion 72

4.6. Closure 81 
Chapter 5: Experimental studies of an energy harvester with adjustable resonance

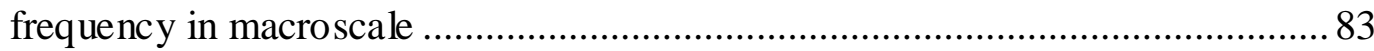

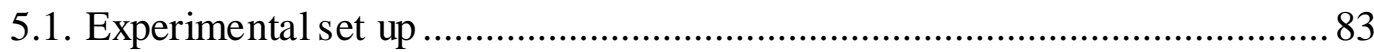

5.2. Characterization of electromagnetic force ................................................. 84

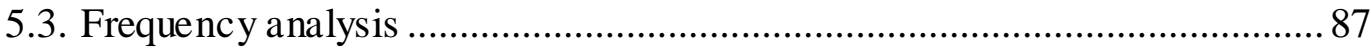

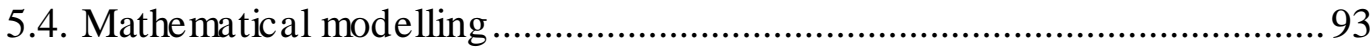

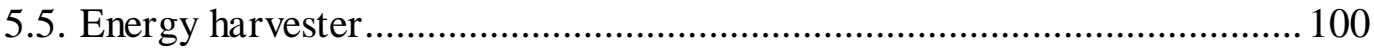

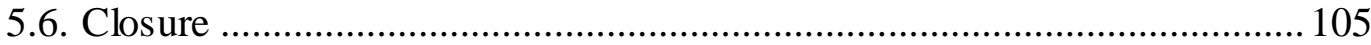

Chapter 6: Conclusions and future work .......................................................... 106

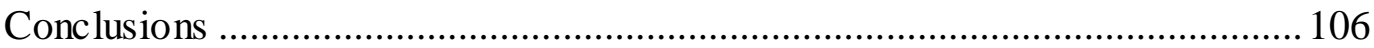

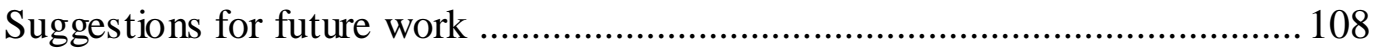

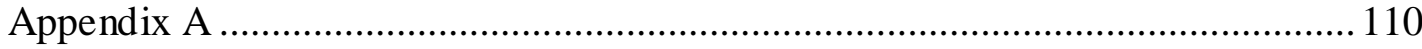

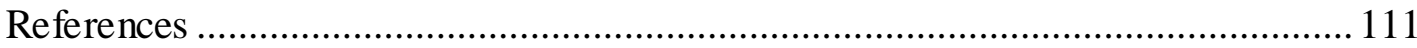




\section{Acknowledgments}

It would not have been possible to write this doctoral thesis without the help and support of the kind people around me, to only some of whom it is possible to give particular mention here.

I would like to thank my supervisors, Dr. Hamed Haddad-Khodaparast and Professor Sondipon Adhikari for all their contributions of time and ideas to make my Ph.D. experience productive and stimulating. Besides my supervisors, I would like to thank Professor Michael Friswell for his insightful comments and ideas during my Ph.D. studies. I acknowledge the financial support from the Swansea University, United Kingdom through the award of the Zienkiewicz scholarship.

Most importantly, I would like to thank my parents and wife, who had always supported me and kept me focused. Their encouragement, sacrifice and never-ending kindness made everything easier to achieve. Indeed, they were my major source of strength and support. 


\section{List of Figures}

Figure 1.1 Overview of MEMS [1] .................................................................. 1

Figure 1.2 Schematic of MEMS components ..........................................................

Figure 1.3 Application of MEMS technology in (a) biomedical and (b) aerospace .........3

Figure 1.4 The market breakdown forecast of MEMS devices from 2012 to 2018 ..........4

Figure 1.5 The direct piezoelectric effect causes certain crystalline materials to generate an electric charge due to the mechanical strain [28]. 6

Figure 1.6 The relationship diagram of piezoelectric materials [29] ...........................6

Figure 1.7 (a) The 33-mode and (b) 31-mode piezoelectric stress driven generator configurations [28].

Figure 1.8 Piezoelectric bimorph cantilever constructions: (a) parallel and (b) series connections of the piezoelectric layers [28].

Figure 1.9 Piezoelectric unimorph cantilever constructions: (a) 31-mode and (b) 33-mode [28].

Figure 1.10 Faraday's law of electromagnetic induction. 9

Figure 1.11 Common types of electromagnetic energy harvesting configurations (a) moving coil with static magnet and (b) moving magnet with static coil [28].

Figure 1.12 (a) The charge-constrained and (b) the voltage-constrained energy cycle. 12

Figure 1.13 Two different types of electrets for electret based electrostatic harvesters (a)

dipole orientation and (b) charge injection.

Figure 2.1 Bimorph piezoelectric energy harvester configurations, (a) series and (b) parallel connection of piezoceramic layers and (c) their cross-sectional view [90] .......24

Figure 2.2 Electrical representation of a piezoceramic layer connected to a resistor. ....27 
Figure 2.3 Electrical circuit representing the series connection of the piezoceramic layers.

Figure 2.4 Electrical circuit representing the parallel connection of the piezoceramic layers.

Figure 2.5 Output voltage of the harvester for a broad range of load resistance 33

Figure 2.6 Output voltage of the harvester for a broad range of load resistance 34

Figure 2.7 Output voltage of the harvester for a broad range of load resistance 35

Figure 2.8 Variation of the tip deflection and output voltage with load resistance for excitations at the short-circuit and open-circuit resonance frequencies.

Figure 2.9 Variation of peak power with load resistance for excitations at (a) the shortcircuit and (b) open-circuit resonance frequencies.

Figure 2.10 Variation of peak power with load resistance for different excitation frequencies

Figure 3.1 Schematic of the proposed energy harvester.

Figure 3.2 Variation of the (a) pull in voltage and (b) the electrostatic force with the air gap between the electrodes $\left(g_{0}\right)$. 48

Figure 3.3 Variation of the natural frequency with electrostatic voltage for different air gaps when $L_{e}=0.5 L$

Figure 3.4 Displacement frequency response curve for different order of nonlinearity.50

Figure 3.5 Displacement frequency response curve 51

Figure 3.6 (a)Variation of the piezoelectric peak power with load resistance for different frequencies of base excitation (b) Variation of the optimal resistance for different frequencies of base excitation 52

Figure 3.7 Variation of the piezoelectric peak power with frequency of base excitation 
Figure 3.8 (a) Frequency range of the harvester based on increasing DC voltage (b)

Voltage range for different lengths of electrodes to cover the frequency range

Figure 3.9 Variation of the piezoelectric peak power with the frequency of base excitation.

Figure 3.10 Variation of the instantaneous electrostatic power with time when $L_{e}=$ $0.01 \mathrm{~L}$ and $\Omega=253 \mathrm{~Hz}$

Figure 3.11 (a) Variation of the piezoelectric peak power with load resistance for a linear harvester (b) Displacement frequency response curve considering optimal resistance ..57

Figure 3.12 Variation of the piezoelectric peak power with DC voltage and excitation frequency $\left(L_{e}=0.5 \mathrm{~L}\right)$. .58

Figure 3.13 Variation of the piezoelectric peak power by changing load resistance and applied voltage at 70 and $80 \mathrm{~Hz}$

Figure 3.14 (a) Frequency range of the harvester based on increasing DC voltage (b) Voltage range for covering the frequency range (c) optimal resistance at each frequency 60

Figure 3.15 Design flow chart .60

Figure 4.1 Aleatory and epistemic uncertainty 63

Figure 4.2 Schematic of the proposed energy harvester .67

Figure 4.3 A micro-beam with an arc-shaped comb finger in its (a) unperturbed state and (b) perturbed state. 68

Figure 4.4 (a) Variation of the piezoelectric peak power with load resistance at nominal resonance frequency (b) Displacement frequency response curve with the optimal resistance .73

Figure 4.5 Probability density function of (a) harvested power and (b) resonance frequency. .74 
Figure 4.6 Tuning resonance frequency of microbeam using (a) softening $\left(d_{2}-d_{1}=\right.$

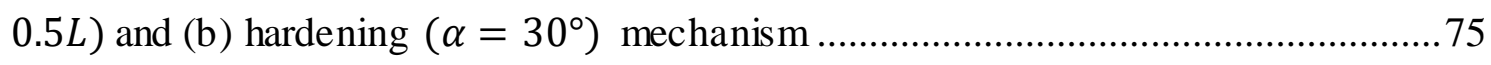

Figure 4.7 (a) Tuning resonance frequency of symmetrical model $\left(g_{01}=g_{02}=40 \mu \mathrm{m}\right)$ (b) comparison of symmetrical and unsymmetrical model $\left(g_{01}=44.6 \mu \mathrm{m}, g_{02}=36 \mu \mathrm{m}\right)$

Figure 4.8 Output power due to the steady state response at $406 \mathrm{~Hz}$ (a) unsymmetrical $\operatorname{model}\left(V_{1}=8 \mathrm{~V}\right)(\mathrm{b}) \operatorname{symmetrical}\left(V_{1}=10.1 \mathrm{~V}\right)$ .77

Figure 4.9 (a) Output power of the harvester on the lower branch $\left(\Omega=406 \mathrm{~Hz}, V_{1}=\right.$ $8 \mathrm{~V}$ ) (b) Moving from the lower branch to the higher by decreasing the voltage.......... 78

Figure 4.10 (a)Variable capacitors in the proposed model (b) Electrical circuit 79

Figure 4.11 Harvested power of samples based on (a) softening and (b) hardening mechanism. .79

Figure 4.12 (a) Applied DC voltage versus harvested power for softening mechanism and (b) hardening mechanism. .80

Figure 4.13 Harvested power of samples before and after applying DC voltage 81

Figure 5.1 Schematic of the proposed model(Top view) .84

Figure 5.2 Tip deflection of the beam versus distance between magnets $\left(V_{D C}=0 \mathrm{~V}\right) . .85$

Figure 5.3 Tip deflection of the beam versus DC voltage applied to the electromagnet 86 Figure 5.4 Tip deflection of the beam versus DC voltage applied to the electromagnet at $\mathrm{D}=25 \mathrm{~mm}$ .87

Figure 5.5 linear model .87

Figure 5.6 FRF of the linear model .88

Figure 5.7 Using electromagnetic device to control the resonance frequency of the beam

Figure 5.8 FRFs for different air gaps between magnets $\left(g_{0}\right)$ 89 
Figure 5.9 FRFs of the beam for different air gaps between magnets $\left(g_{0}\right)$ .90

Figure 5.10 FRFs of the beam for different air gaps between magnets $\left(g_{0}\right)$ 91

Figure 5.11 FRF of the beam for different air gaps between magnets $\left(g_{0}\right)$ 92

Figure 5.12 Top view of the model 93

Figure 5.13 FRF of the linear model: comparison between experimental and theoretical results 96

Figure 5.14 FRF of the beam for different air gaps between magnets (there is no applied voltage to the electromagnets)

Figure 5.15 FRF of the beam $\left(g_{0}=30 \mathrm{~mm}\right)$ 99

Figure 5.16 Proposed energy harvester..... 100

Figure 5.17 FRFs of the linear model with added MFC piezoelectric patches 101

Figure 5.18 voltage divider circuit 102

Figure 5.19 Voltage output FRFs of the piezoelectric patches. 102

Figure 5.20 Proposed energy harvester with frequency tuning mechanism 103

Figure 5.21 Voltage output FRF of the piezoelectric patches when $V_{D C}=5 \mathrm{~V}$ 103 


\section{List of Tables}

Table 2.1 Geometrical and material properties of the MEMS harvester

Table 2.2 Geometrical and material properties of the harvester [90]

Table 3.1 Geometrical properties of the microbeam and piezoelectric layers .56

Table 4.1 Geometrical properties of the arch-shaped electrodes. 72

Table 4.2 Parameters most sensitive to manufacturing uncertainties[102] .72

Table 5.1 Geometrical and material properties of the beam and tip magnets 84

Table 5.2 Identified values for $K_{0}$ and $K_{n}$ for different initial gaps at $V=0 \mathrm{~V}$ .98

Table 5.3 Identified values for $K_{0}$ and $K_{n}$ for different voltages at $g_{0}=30 \mathrm{~mm}$ . .99

Table 5.4 Geometrical and material properties of the MFC piezoelectric patches 100 


\section{List of Symbols and Abbreviations}

$d_{3 i} \quad$ Piezoelectric material performance coefficient $(\mathrm{i}=1,3)$

$V_{o c} \quad$ Open circuit voltage of the piezoelectric layer

$\sigma_{i j} \quad$ Applied stress to the piezoelectric layer

$g_{e} \quad$ Gap between electrodes

$\varepsilon_{0} \quad$ Permittivity of vacuum

$\varepsilon \quad$ Dielectric permittivity

$\varepsilon_{r} \quad$ Relative dielectric constant

$\Phi \quad$ Magnetic flux

$N \quad$ Number of turns in coil

$A_{i} \quad$ enclosed area over the $i$ th wire loop

$B \quad$ Magnetic flux density over the $i$ th wire loop

$V_{S} \quad$ Surface potential

$d_{e} \quad$ Thickness of the electret

$M_{b} \quad$ Internal bending moment

$w_{\text {rel }} \quad$ Vertical displacement of the beam relative to its base

$m \quad$ Mass per unit length of the beam

$M_{t} \quad$ Tip mass

$w_{b} \quad$ Base displacement

$\delta(x) \quad$ Dirac delta function

$H(x) \quad$ Heaviside function

$\begin{array}{ll}c_{a} & \text { Viscous air damping coefficient }\end{array}$

$h_{\tilde{S}} \quad$ Thickness of the beam

$h_{\tilde{p}} \quad$ Thickness of each piezoceramic layer

$b \quad$ Width of the cantilever beam and each piezoceramic layer

$T_{\tilde{S}} \quad$ Stress components in the beam 
$T_{\tilde{p}} \quad$ Stress components in the piezoceramic

$E_{\tilde{S}} \quad$ Elastic modulus of the beam

$\bar{c}_{11}^{E} \quad$ Elastic modulus of the piezoceramic layers at a constant electric field

$E_{3} \quad$ Electric field component in the poling direction

$S \quad$ Axial strain

$s_{11}^{E} \quad$ Elastic compliance

$\bar{e}_{31} \quad$ Effective piezoelectric stress-constant

EI Bending stiffness term

$M_{b}^{S} \quad$ Internal bending moment for series connection

$M_{b}^{p} \quad$ Internal bending moment for parallel connection

$\vartheta_{s} \quad$ Backward coupling term for series connection

$\vartheta_{p} \quad$ Backward coupling term for parallel connection

$\rho_{\tilde{p}} \quad$ Density of the piezoceramic

$\rho_{\tilde{S}} \quad$ Density of the beam

$D_{3} \quad$ Electric displacement component

$A_{e} \quad$ Area of the electrode

$\bar{\varepsilon}_{33}^{\tilde{S}} \quad$ Permittivity component at constant strain and plane stress assumption for the beam

$h_{\tilde{p} c} \quad$ Distance between the center of each piezoceramic layer and the neutral axis

$R \quad$ Load resistance

$C_{\tilde{p}} \quad$ Piezoelectric internal capacitance

$i_{\tilde{p}}^{S} \quad$ Output current of the piezoceramic layers for series connection

$i_{\tilde{p}}^{p} \quad$ Output current of the piezoceramic layers for parallel connection

$\theta_{p} \quad$ Electrical coupling coefficient of the single-mode approximation for parallel connection 
$P_{\text {ave }} \quad$ Average harvested power from the piezoelectric layers

$P_{\max } \quad$ Peak harvested power from the piezoelectric layers

$A_{r} \quad$ Modal amplitude constant

$I_{t} \quad$ Tip mass moment of Inertia

$\omega_{r}^{s c} \quad$ Short-circuit resonance frequency

$\omega_{r}^{o c} \quad$ Open-circuit resonance frequency

$F_{\text {es }} \quad$ Electrostatic force between electrodes

$g_{0} \quad$ Air gap between electrodes

$K_{m} \quad$ Mechanical stiffness

$K_{e} \quad$ Electrical stiffness

$K_{n i} \quad$ Coefficients of the nonlinearity $(\mathrm{i}=1,2,3,4)$

$L_{e} \quad$ Length of the electrodes

$z_{0} \quad$ Amplitude of the base excitation

$\Omega \quad$ Frequceny of the base exciatation

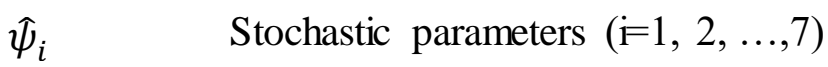

$V_{1} \quad$ DC voltage applied to the straight electrodes

$V_{2} \quad$ DC voltage applied to the arc-shaped electrodes

$F_{f} \quad$ Follower force

$E I_{C} \quad$ Bending stiffness of the bimorph piezoelectric harvester

$m_{C} \quad$ Mass per unit length of the bimorph piezoelectric harvester

$h_{r} \quad$ Thickness of arc-shape electrode

$g_{r} \quad$ Radial air gap

$h_{0} \quad$ Thickness of the permanent magnets

$D_{0} \quad$ Diameter of the permanent magnets

$E_{0} \quad$ Young's modulus of permanent magnets

$\rho_{0} \quad$ Density of the permanent magnets 
$F_{M} \quad$ Magnetic force

$M_{s} \quad$ Mass of the ICP sensors

MEMS Micro-Electro-Mechanical Systems

IC Integrated Circuit

HARM High Aspect-Ratio Micromachining

MST Micro-Systems Technology

AFM Atomic Force Microscope

IDDSs Implantable Drug Delivery Systems

AE Acoustic Emission

WSNs Wireless Sensor Nodes

SHM Structural Health Monitoring

PDF Probability Density Function

DST Dempster-Shafer theory

MCS Monte Carlo Simulation

LHS Latin Hypercube Sampling 


\section{Chapter 1: Introduction}

First chapter of this thesis gives a brief overview of microelectromechanical system (MEMS) and its wide range of applications across different fields. Based on the application of MEMS devices, supplying power for these devices is one of key issues. Generally, these devices need small amount of power to operate which can be harvested from ambient sources of energy in our environment. There are different energy harvesting technologies which will be discussed in this chapter.

\subsection{Microelectromechanical systems (MEMS)}

Microelectromechanical systems (MEMS) consist of a high-class technology which uses sophisticated manufacturing methods to create small integrated devices that combine silicon-based microelectrical and mechanical components. These devices range in size from a few micrometres to millimetre and can be fabricated using integrated circuit (IC) batch processing techniques. The fabrication of MEMS devices includes bulk and surface micromachining, as well as high aspect-ratio micromachining (HARM) which can selectively remove parts of the silicon or add different layers to form the mechanical and electromechanical components.

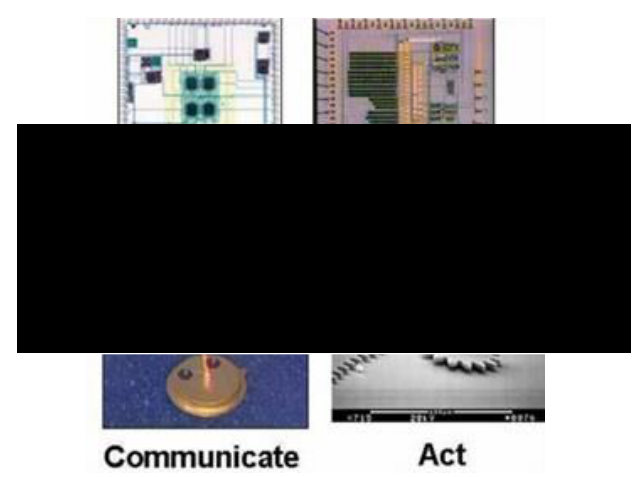

Figure 1.1 Overview of MEMS [1]

MEMS devices can sense, think, act and communicate in micro scale, and generate effects on the macro scale. The interaction of electronics, mechanics, light or fluids working 
together makes up a microelectromechanical system, or MEMS, which is an acronym that originated in the United States. It is also referred to as Microsystems Technology (MST) in Europe and Micromachines in Japan. As Figure 1.2 shows, MEMS consist of mechanical microstructures, microsensors, microactuators and microelectronics which have been integrated into the same silicon chip.

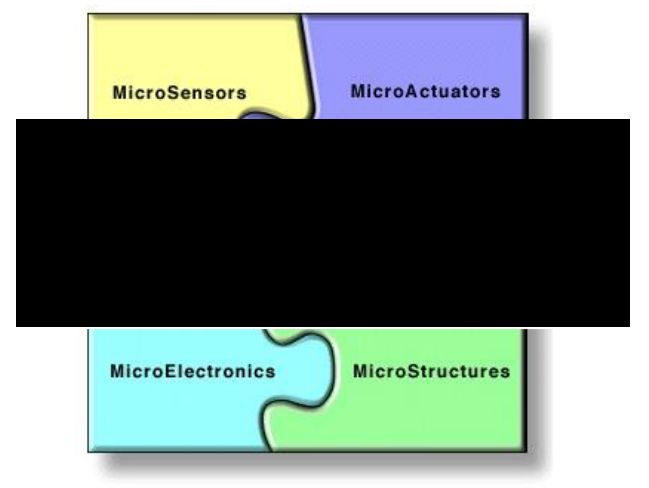

Figure 1.2 Schematic of MEMS components

The changes in the system's environment are measured by microsensors. Afterwards, microelectronics process the measured information and signal the microactuators to react and create some form of change to the environment. From originating in a very early version in the early 1950's, this technology began appearing in numerous commercial products by the mid-1990's. MEMS devices are generally classified according to their actuation mechanisms. Actuation mechanisms for MEMS vary depending on their suitability to the application at hand. The most common actuation mechanisms are electrostatic, pneumatic, thermal, and piezoelectric. Electrostatically actuated devices form a broad class of MEMS devices due to their simplicity, as they require few mechanical components and small voltage levels for actuation. These devices are influenced by instability, which is known as pull-in phenomenon in MEMS literature. In pull-in voltage, the elastic restoring force can no longer resist the opposing electrostatic force, thereby leading to the collapse and failure of the structure. Hence, pull-in instability is a major structural safety concern for MEMS structural design.

On the other hand, MEMS devices are characterized by low cost (due to batch fabrication), small size and lightweight. These features make them applicable in many places where large devices do not fit. 


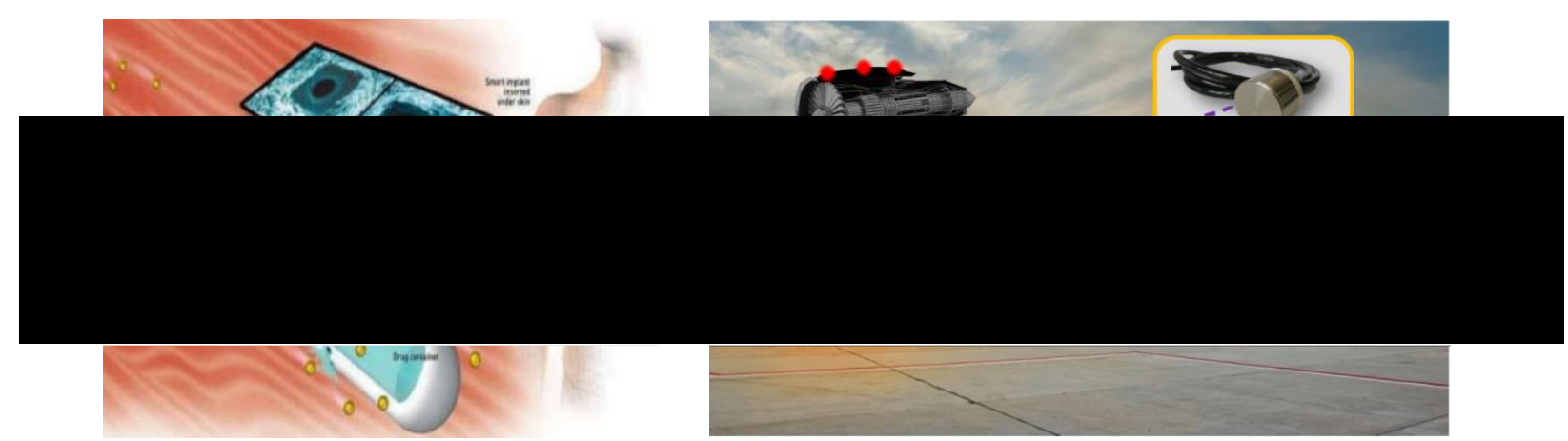

(a)

(b)

Figure 1.3 Application of MEMS technology in (a) biomedical and (b) aerospace

Through advances in MEMS material and processing technology, MEMS devices have gained more popularity in modern technologies, such biomedical $[2,3]$, aerospace $[4,5]$, automotive [6,7], wireless and communications [8,9], atomic force microscope (AFM) $[10,11]$, detection of single electron spin [12], sensing sequence-specific DNA [13], mass sensors [14, 15], chemical sensors [16] and pressure sensors [17, 18]. For example, in biomedical application, there are microscale implantable drug delivery systems (IDDSs) which can be used to control drug delivery parameters, providing better disease control through more accurate, targeted and less painful drug delivery. Also, in aerospace application, by using MEMS Acoustic Emission (AE) sensors we can detect any failures occurring in materials and structures.

Figure 1.4 shows the market breakdown forecast of MEMS devices from 2012 to 2018. Based on this forecast, the use of MEMS chips will top \$22 billion by 2018 [19]

In addition, MEMS devices need small amounts of power to operate, which not only reduces the operational cost but also paves the way for developing long-life and selfpowered devices that can harvest the requested power from the environment. Therefore, harvesting a small amount of energy from our environment can be used to power microdevices which are able to do extraordinary tasks. Moreover, more efficient use of ambient sources of energy requires different kinds of sensing microsystems to control the harvesting procedure which proliferate the application of MEMS devices in energy harvesting areas. 


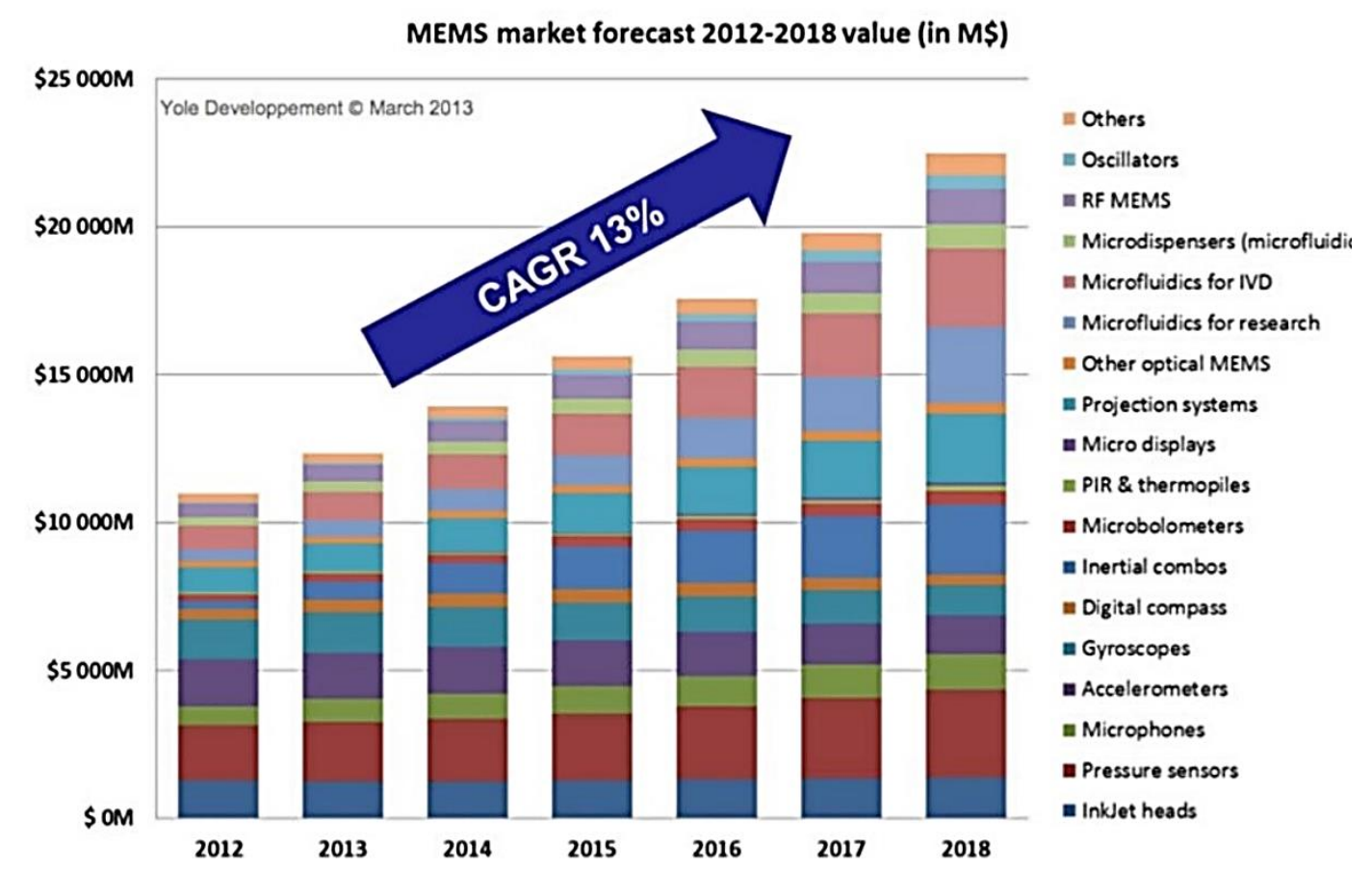

Figure 1.4 The market breakdown forecast of MEMS devices from 2012 to 2018

\subsection{Energy harvesting technology}

Nowadays, the proliferation of sensor nodes and electronic devices means the provision and efficient use of power is a key concern. In electronic devices the batteries are considered one of largest contributing elements to both size and cost. Additionally, due to the limited capacity of batteries, they have a limited useful lifespan before some sort of maintenance is needed. Furthermore, using battery-less and self-powered sensors can be highly beneficial to the environment otherwise disposal of millions of dead batteries will be a huge environmental problem. Therefore, techniques which can harvest energy from the environment are considered as a viable solution for powering sensor nodes and electronic devices.

In our environment there are a variety of ambient energy sources such as solar, man-made radiation, temperature, wind and vibration. Converting ambient wasted energy into small amount of electrical energy can power many useful low-powered MEMS devices in different applications, ranging from wireless sensor nodes (WSNs) to structural health monitoring (SHM) sensors. These devices require low power, in the range of microwatts. Solar energy from solar cells is the most developed form of energy harvesting and it can be ideal where high ambient light levels are reliably available. Where they are not, 
ambient man-made radiation, temperature gradients and vibration are potential alternatives.

Using vibration sources has gained more popularity due to its high availability in various environments. Generally, vibration-based energy harvesters convert ambient kinetic energy into electricity. The most explored application of MEMS in energy harvesting technology is related to harvesting power from wasted vibration energy, something to which integrated electro-mechanical technology is perfectly suited. There are three main categories of ambient vibration sources: household electrical appliances, machineries, and moving vehicles. As stated by William and Yates [20] in their early work on vibration-based energy harvesters for microsystems, the most common types of energy transduction mechanisms are piezoelectric [21, 22], electromagnetic [23, 24] and electrostatic $[25,26]$. The following sections give an overview of the principles of these different kinds of vibration-based energy harvesters.

\subsubsection{Piezoelectric energy harvesters}

Piezoelectric effect is a unique property of certain crystalline materials which was first discovered by the brothers Pierre Curie and Jacques Curie in 1880. In these kind of materials, there is a linear electromechanical interaction between the mechanical and the electrical state. As shown in Figure 1.5, by applying mechanical force to these materials, the crystals become electrically polarized. In fact, any compression or tension generated can shift the positive and negative charge centres and generate electrical voltage which is proportional to the applied force. Alternatively, by applying an electrical field to the crystal, it experiences mechanical strain based on the strength and polarity of the electrical field. Based on the structural characteristics of materials, all crystals obey the 32-point groups [27]. It indicates that there are only 32 possible combinations of symmetrical elements. Of these 32 groups, 11 crystal classes are centrosymmetric and therefore have no polar properties. 


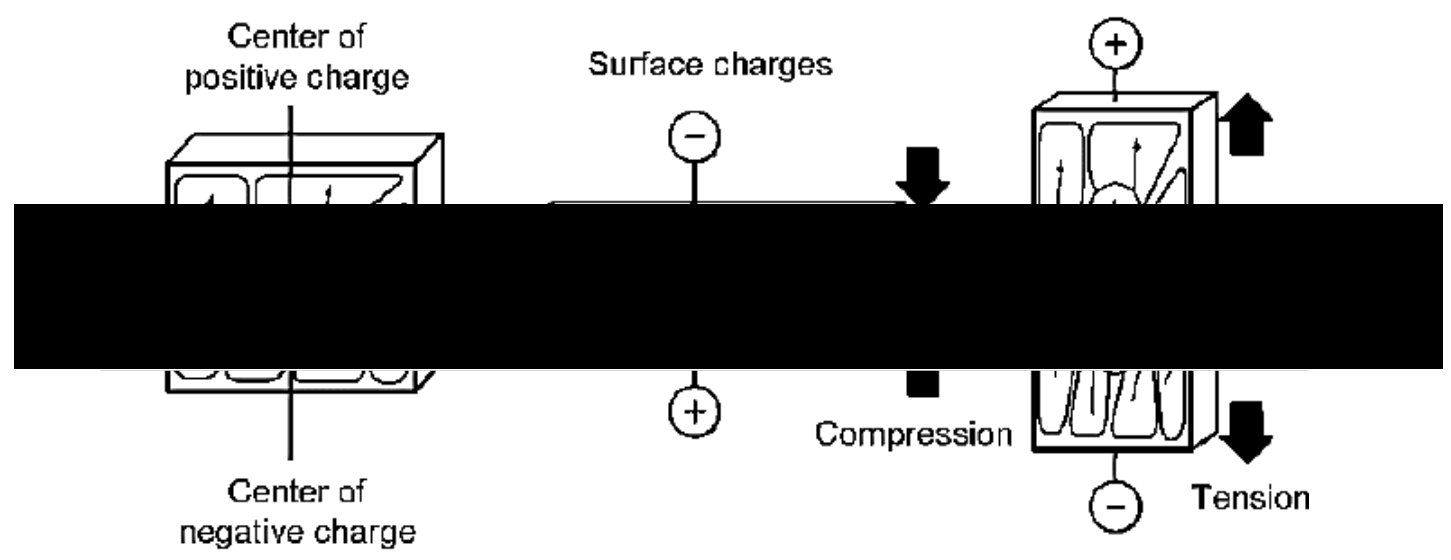

Figure 1.5 The direct piezoelectric effect causes certain crystalline materials to generate an electric charge due to the mechanical strain [28].

Of the remaining 21 non-centrosymmetric classes, 20 classes exhibit electrical polarity when a stress is applied. Out of these 20 classes, 10-point groups belong to polar materials which possess electric dipole moments without being exposed to an electrical field. These crystals can be spontaneously polarized, and polarization can be compensated through external or internal conductivity or twinning or domain formation.

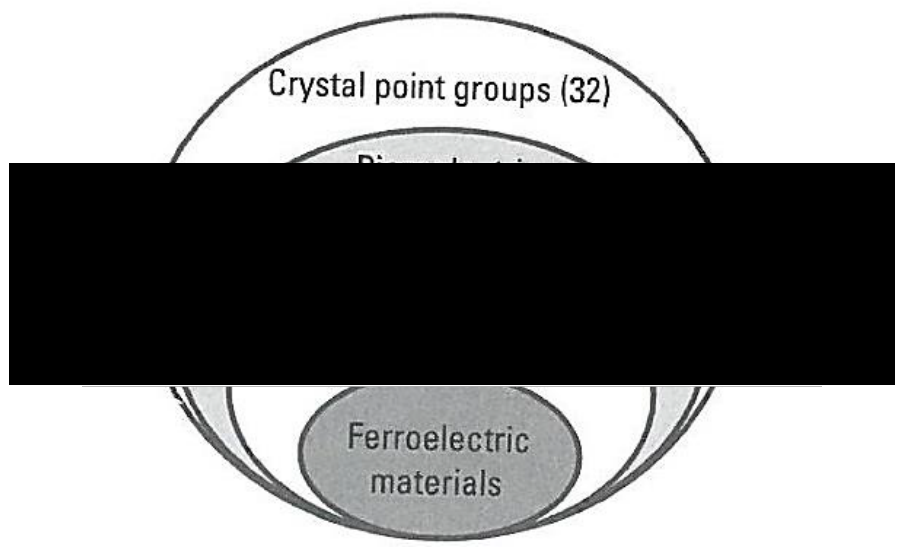

Figure 1.6 The relationship diagram of piezoelectric materials [29].

Among different polar material, there is a special class of materials that are called ferroelectric, such as lead zirconate titanate $(\mathrm{PZT})$, zinc oxide $(\mathrm{ZnO})$ and barium titanate $\left(\mathrm{BaTiO}_{3}\right)$ which have a high electromechanical coupling. These materials exhibit an internal spontaneous polarization within a certain temperature range. By applying a sufficiently large external electric field, the internal spontaneous polarization can be reversed. 
Most piezoelectric materials exhibit a polar axis, and the energy harvesting performance is affected by the direction of the applied force relative to the polar axis. For a ferroelectric ceramic or polymer, the polar axis depends on the direction of the applied force. Generally, the polar axis is referred to the "3" direction and due to the symmetry, other directions at right angles to this axis are equivalent and can be referred to as the "1" directions. As illustrated in Figure 1.7, depending on the direction of stress (i.e. along the polar axis or at right angles to it), there are two common piezoelectric energy harvesting configurations, 33-mode and 31-mode [28].

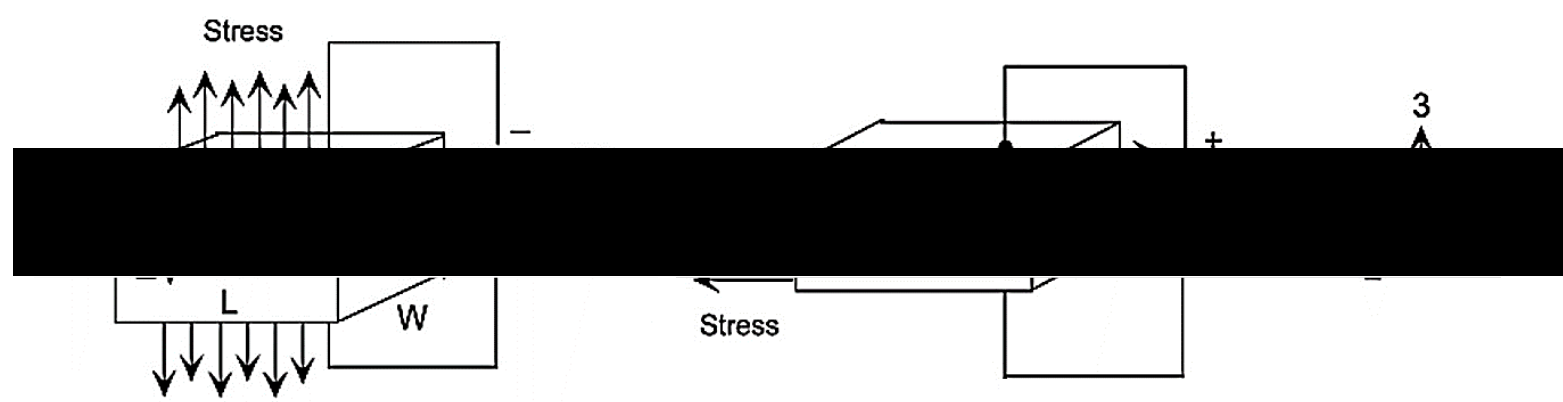

(a)

(b)

Figure 1.7 (a) The 33-mode and (b) 31-mode piezoelectric stress driven generator configurations [28].

Piezoelectric material performance is quantified by the piezoelectric coefficient $\left(d_{3 i}\right)$ which is the ratio of the open circuit charge density to the applied stress (in unit of $\mathrm{C} / \mathrm{N}$ ). Typically, in the piezoelectric materials a $d_{33}$ coefficient is higher than $d_{31}$ coefficient. However, harvesting energy in 31-mode needs the use of large strain in the 1-direction and therefore is commonly used in vibration-based energy harvesting.

Generally, piezoelectric energy harvester design is based on the form of bimorph or unimorph cantilevers [30-33]. Figure 1.8, shows the most common 31-mode uniform bimorph piezoelectric energy harvester configurations. As shown in this figure, two separate piezoelectric layers are bonded to the cantilever beam and the structure operates in a bending mode. Therefore, the instantaneous average bending strains in the elements of the top and bottom layers have the opposite sign (i.e. one is in compression while the other is in tension). Consequently, if the top and bottom layers are poled oppositely in the thickness direction, the induced electric voltage by each layer will be added and the configuration called series polling. Conversely, if the top and bottom layers are polled in 
the same direction and the electrodes are wired properly, the output current generated by each layer will be added and the configuration termed as parallel polling. Therefore, series polling produces a larger output voltage whereas parallel polling produces a larger output current under optimal conditions.

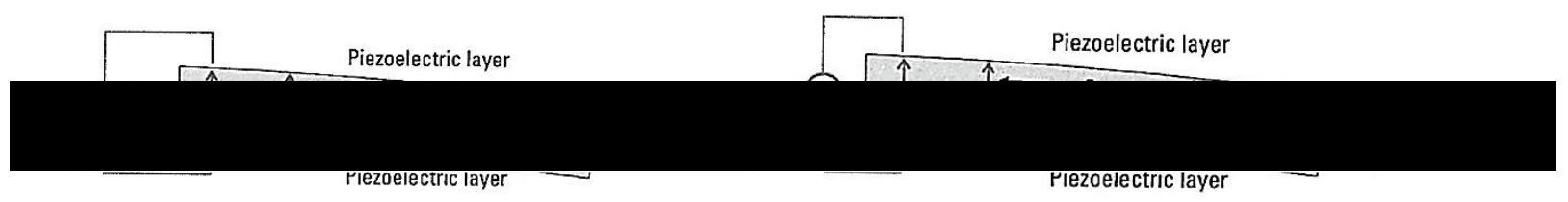

(a)

(b)

Figure 1.8 Piezoelectric bimorph cantilever constructions: (a) parallel and (b) series connections of the piezoelectric layers [28].

The unimorph cantilever configuration is another basic form of vibration-based piezoelectric energy harvester. As shown in Figure 1.9, in 31-mode, the piezoelectric layer is sandwiched by the top and bottom electrodes, while in 33-mode, the electrodes are on top of the piezoelectric layer with an interdigital finger pattern. In both cases, the piezoelectric layer is bonded on top of the cantilever beam.

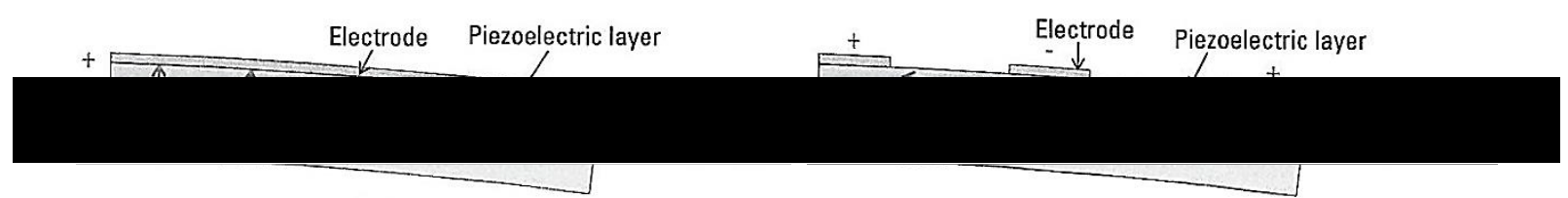

(a)

(b)

Figure 1.9 Piezoelectric unimorph cantilever constructions: (a) 31-mode and (b) 33mode [28].

The open circuit voltage of the piezoelectric layer $V_{o c}$ is given as [28]

$$
V_{o c}=\frac{d_{i j}}{\varepsilon_{r} \varepsilon_{0}} \sigma_{i j} g_{e}
$$

In equation $1.1, \sigma_{i j}$ is the applied stress, $d_{i j}$ is the piezoelectric coefficient, $g_{e}$ is the gap between electrodes and $\varepsilon_{0}$ and $\varepsilon_{r}$ are permittivity of vacuum and the relative dielectric constant, respectively. The performance of a unimorph cantilever piezoelectric harvester is mostly dependent on the type of piezoelectric mode. Considering the most common type of piezoelectric materials PZT as an example and the same configuration parameters, 
the output voltage of an energy harvester for 33-mode is expected to be greater than that of 31-mode. On the other hand, the output voltage is proportional to the distance between electrodes $\left(g_{e}\right)$ for 31-mode and to the distance between fingers for a 33-mode device. In microelectromechanical system (MEMS), the piezoelectric layers are very thin, therefore the distance between electrodes in 31-mode is shorter than in 33-mode. Consequently, the 33-mode can generate higher voltage output, while 31-mode has the advantage of generating larger output current. Considering the output power based on the production of voltage and current, a better performance in 31-mode in comparison with 33-mode was reported by Lee et al. [34]. A similar comparison was made by Kim et al. [35] and they concluded that by optimizing the interfinger electrode (IDE), higher power and voltage could be achieved from the 33-mode device.

\subsubsection{Electromagnetic energy harvesters}

Electromagnetism has been used to produce electricity since the 1930s. Over the last decade, different kinds of micro/macro electromagnetic harvesters have been proposed with output voltages ranging from microwatts to watts. The basic concept for most of these harvesters is based on Faraday's law of electromagnetic induction. As shown in Figure 1.10, the voltage, or electromotive force (EMF), is induced in the conductive loops of wire due to the change in magnetic flux.

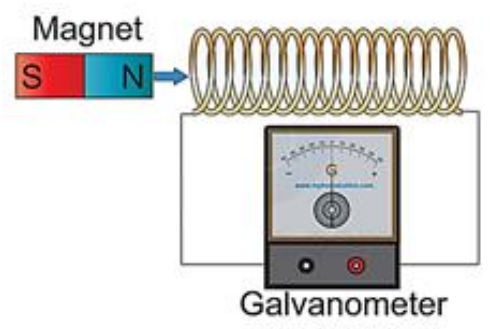

Figure 1.10 Faraday's law of electromagnetic induction.

Generally, electromagnetic energy harvesters consist of a magnet, a coil, a mechanical spring, damping and a frame. The spring supports either the coil or magnet and allows the relative movement in the device. The induced voltage in an $N$ turn coil is given as [28] 


$$
\varepsilon=-\frac{d \Phi}{d t}=-\sum_{i=1}^{N} \int B d A_{i}
$$

where $\Phi$ is the total magnetic flux of the $N$ turn coil, $A_{i}$ and $B$ are the enclosed area and the magnetic flux density over the $i$ th wire loop, respectively. It is noteworthy that electromagnetic induction can be induced either by changing the area at a constant magnetic field or changing the magnetic field at a constant area [28]. Figure 1.11 shows two common types of electromagnetic energy harvester configurations including the change of the magnetic flux: the moving coil with a static magnet (Figure 1.11a) and the moving magnet with a static coil (Figure 1.11b). In both configurations, the electrical current will be induced as a result of changing the magnetic flux across the coil.

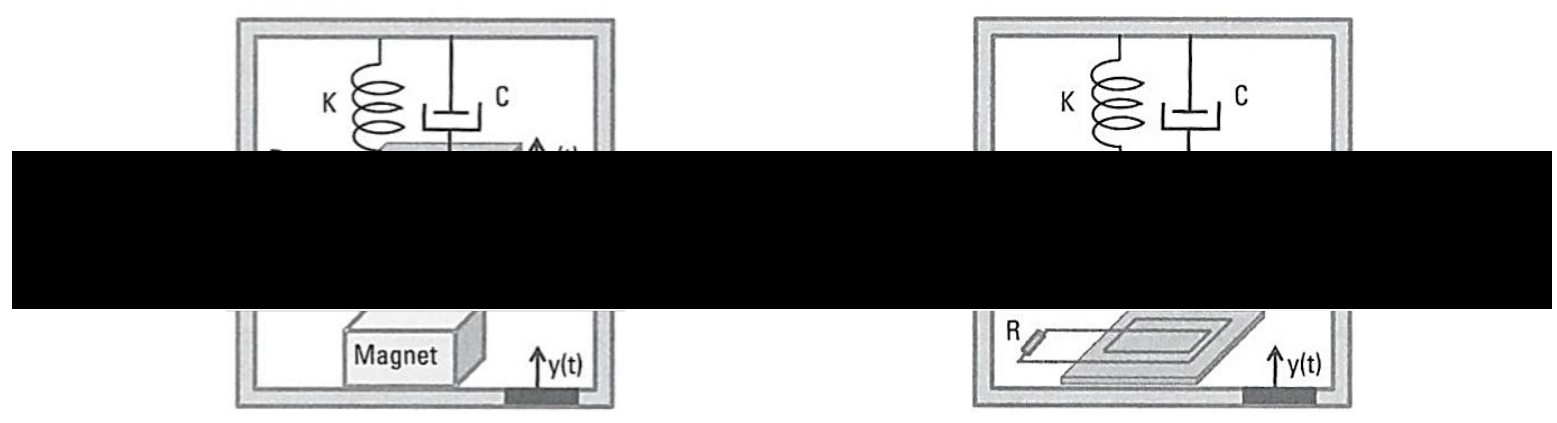

(a)

(b)

Figure 1.11 Common types of electromagnetic energy harvesting configurations (a) moving coil with static magnet and (b) moving magnet with static coil [28].

\subsubsection{Electrostatic energy harvesters}

Electrostatic energy harvesters can be considered a good choice where miniaturization is beneficial. Since the standard MEMS and packing techniques for the fabrication of electrostatic transducers has been well established, capacitive-based electrostatic energy harvesters can be fabricated in large numbers and at low cost. The principle of electrostatic energy harvester is based on a variable capacitor and depending on how the capacitance is changed, capacitive-based electrostatic energy is classified into three types including in-plane gap closing which varies the gap between electrode fingers, in-plane overlap which varies the overlap area between electrodes, and out-of-plane gap closing 
which varies the gap between two electrodes [36-38]. Generally, electrostatic energy harvesters can be divided into two categories:

- Electret-free electrostatic converters

- Electret-based electrostatic converters

In Electret-free electrostatic converters, some controlled switches are required to change the capacitor's condition discontinuously through conversion cycle and must be synchronized with the capacitance variation. However, in Electret-based electrostatic converters, the harvester can convert mechanical power into electricity directly.

\subsubsection{Electret-free electrostatic harvesters}

Electret-free electrostatic harvesters are passive structures that require an energy cycle to convert mechanical energy into useful power. There are two types of energy conversion cycles, charge-constrained and voltage-constrained modes $[37,39]$. In the chargeconstrained cycle, while the charge on the capacitor is constrained, decreasing the capacitance increases the voltage. However, in the voltage-constrained cycle, the voltage across the capacitor will be constrained and by decreasing the capacitance, charge is moved from the capacitor [21].

\subsection{Charge-constrained cycle}

The charge-constrained conversion cycle in the Q-V diagram has been shown in Figure 1.12a. Cycle A-B-C-A represents the conversion cycle. At the beginning, the capacitance reaches its maximum under a given voltage of $V_{\min }$ (Path A-B). The energy stored in the system can be written as

$$
E_{B}=\frac{1}{2} C_{\max } V_{\min }^{2}
$$

Then with the aid of a switch, the electrodes are electrically isolated and the charge on the electrodes is kept constant. Therefore, from point $\mathrm{B}$ to point $\mathrm{C}$, the capacitance is decreased to $C_{\min }$. By decreasing the capacitance at the constant charge, the effective potential voltage increases from $V_{\min }$ to $V_{\max }$. The stored energy at point $\mathrm{C}$ can be expressed as 


$$
E_{C}=\frac{1}{2} C_{\min } V_{\max }^{2}
$$

In the last step of the energy conversion cycle, the capacitance is discharged, and the electric charges are removed from the harvester. Therefore, the total harvested energy in one conversion cycle is

$$
E_{\text {total }}=E_{C}-E_{B}=\frac{1}{2}\left(C_{\min } V_{\text {max }}^{2}-C_{\text {max }} V_{\text {min }}^{2}\right)
$$

Since the capacitor works in the charge-constrained cycle, the charges at point $\mathrm{B}$ and $\mathrm{C}$ are the same.

$$
Q_{\max }=C_{\min } V_{\max }=C_{\max } V_{\min }
$$

Using equation 1.6, the total harvested energy in one energy cycle can be written as

$$
E_{\text {total }}=\frac{1}{2} V_{\text {min }}^{2} \frac{C_{\text {max }}}{C_{\text {min }}}\left(C_{\text {max }}-C_{\text {min }}\right)=\frac{1}{2} V_{\text {max }} V_{\text {min }} \Delta C
$$

\subsection{Voltage-constrained cycle}

Figure $1.12 \mathrm{~b}$ shows another energy conversion cycle named the voltage-constrained cycle. The energy conversion starts when the capacitance reaches to its maximum at a high voltage $\left(V_{\max }\right)$.

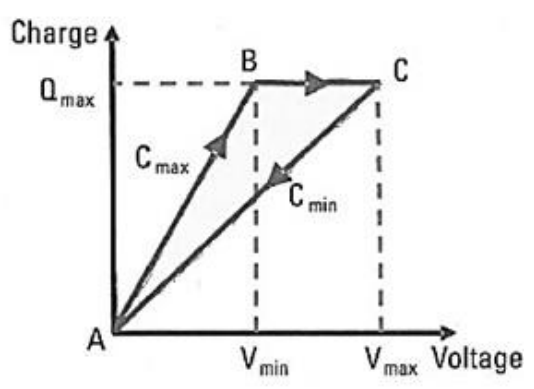

(a)

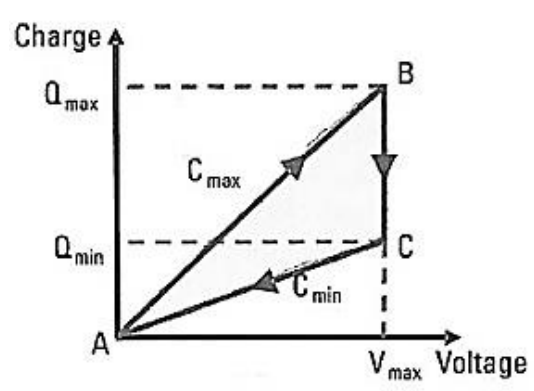

(b)

Figure 1.12 (a) The charge-constrained and (b) the voltage-constrained energy cycle. 
Then by decreasing the capacitance to the minimum value of $C_{\min }$ at the constant voltage, the charge decreases from $Q_{\max }$ to $Q_{\min }$. In this step, the generated charges $\left(Q_{\max }-\right.$ $Q_{\min }$ ) will return to the voltage source or an external load. The net harvested energy per cycle can be expressed as

$$
E_{\text {total }}=\frac{1}{2} V_{\max }^{2}\left(C_{\max }-C_{\min }\right)=\frac{1}{2} V_{\max }^{2} \Delta C
$$

In both conversion cycles, the converted energy will be reduced because of the losses in the conversion electric circuit. Considering the same values for $C_{\max }$ and $C_{\text {min }}$, a voltageconstrained cycle is more efficient than a charge-constrained conversion cycle [40]. On the other hand, the voltage-constrained cycle requires a voltage source $\left(V_{\max }\right)$ to be connected in all steps whereas the charge-constrained conversion cycle only needs a voltage source $\left(V_{\min }\right)$ to be charged at the beginning of the conversion cycle.

\subsubsection{Electret-based electrostatic harvesters}

The conversion cycle in electret-based electrostatic harvesters is quite similar to the electret-free electrostatic harvesters. The only difference is related to the electret layer that is added to the variable capacitor and polarizes it. Generally, electrets are dielectric materials with a dipole polarization or quasi-permanent electrical charge. They can provide an electrostatic field for tens of years [41]. There are different materials such as Teflon, $\mathrm{SiO}_{2}$ and CYTOP, which can be used as electrets in electrostatic energy harvesters. The polarization of electrets can be obtained either by charge injection or dipole orientation (See Figure 1.13).

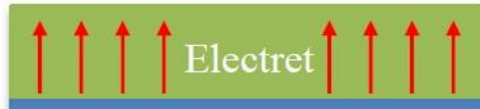

Electrode

(a)

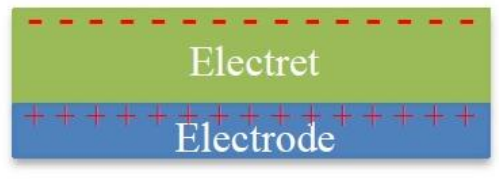

(b)

Figure 1.13 Two different types of electrets for electret based electrostatic harvesters (a) dipole orientation and (b) charge injection.

The electret layers are added to either one or both the plates of the capacitor and are able to polarize the harvesters throughout their lives, thereby enabling the direct mechanical 
to electrical conversion without any external source [42-44]. Based on the polarization method, electrets can be classified into two categories: dipole oriented electrets and charge injection electrets (See Figure 1.13). The fabrication procedures of oriented-dipole and real-charge electrets can be studied in [45] Due to the charge injection or dipole orientation, electrets have a surface potential $\left(V_{s}\right)$ which can be expressed as

$$
V_{s}=\frac{\sigma d_{e}}{\varepsilon \varepsilon_{0}}
$$

In which, $\varepsilon$ is the dielectric permittivity, $\varepsilon_{0}$ is the permittivity of vacuum, $\sigma$ is the surface charge density and $d_{e}$ is the thickness of the electret. Therefore, the electret layer can be modelled as a capacitor and its capacitance is given by

$$
C_{e}=\frac{\varepsilon \varepsilon_{0} A}{d_{e}}
$$

\subsection{Review of MEMS vibration-based energy harvesters}

So far, there have been several studies on the MEMS vibration-based energy harvesters and different models have been proposed based on the electromagnetic, piezoelectric, and electrostatic transduction methods.

In 2005 Jeon et al. [46] studied a $170 \mu \mathrm{m} \times 260 \mu \mathrm{m}$ PZT beam power generator that can harvest $1 \mu \mathrm{W}$ power output across a 5.2 $\mathrm{M} \Omega$ resistive load from a $10.8 \mathrm{~g}$ vibration at its resonance frequency of $13.9 \mathrm{kHz}$. Subsequently, a second generation of Piezoelectric Micro Power Generator (PMPG) was proposed by the same group [47]. They considered the effect of proof mass, beam shape and damping on the performance of the system and showed that the maximum harvested power occurs when the resonance frequency of the energy harvester matches the dominant excitation frequency. Based on their results, the maximum power harvesting, can be achieved by having a proof mass as heavy as possible unless it results other adverse effects such as excessive stress or damping.

Marzencki et al. [48], successfully designed and fabricated a thin film AIN cantilever micro-generator, that can generate a power of $0.038 \mu \mathrm{W}$ from a $0.5 \mathrm{~g}$ acceleration at 204 $\mathrm{Hz}$ resonance frequency. However, the output power is limited to low power levels due to the properties of AIN material. In their later works Marzencki et al. [49], improved the power generated by increasing the vibration amplitude and frequency of their device to 4 
$\mathrm{g}$ at $1368 \mathrm{~Hz}$ resonance frequency to generate a power of $1.97 \mu \mathrm{W}$. Muralt et al. [50], designed and fabricated a micro power generator of thin film PZT laminated cantilever with proof mass and inter-digitated electrodes which could generate about $1.6 \mathrm{~V}$ and 1.4 $\mu \mathrm{W}$ when excited under $2 \mathrm{~g}$ at $870 \mathrm{~Hz}$ resonance frequency. Elfrink et al. [51], designed and fabricated a MEMS-based AIN piezoelectric cantilever micro-generator, that can generate an output power of $60 \mu \mathrm{W}$ under $2 \mathrm{~g}$ acceleration at $572 \mathrm{~Hz}$ resonance frequency. Feng et al. [52] investigated a MEMS based PZT cantilever power generator with a proof mass and showed that the system is capable of harvesting $2.16 \mu \mathrm{W}$ from a $1 \mathrm{~g}$ vibration at its resonance frequency of $609 \mathrm{~Hz}$. While the demonstrated power density is quite high, the proof mass was not integrated with the cantilever beam. There is difficulty in fabricating high quality PZT thin films and/or complex process procedures to produce MEMS PZT-based cantilevers with an integrated proof mass at the end tip. A PZT-based micro cantilever with an integrated proof mass was not manufactured until 2007. Renaud et al. [53] reported the fabrication, modelling, and characterization of a MEMS piezoelectric cantilever power generator with an integrated proof mass that can generate an average power of $40 \mu \mathrm{W}$ at $1.8 \mathrm{kHz}$. Shen et al. [54] designed a MEMS piezoelectric energy harvesting device for low vibration frequency and high vibration amplitude environments. They showed that with a beam dimension of $4.8 \mathrm{~mm} \times 0.4 \mathrm{~mm} \times 0.036$ $\mathrm{mm}, 2.15 \mu \mathrm{W}$ power can be harvested at $461.15 \mathrm{~Hz}$. Gu et al. [55] proposed an impactdriven FUC (Frequency Up-Conversion) energy harvesting prototype that is suitable for MEMS implementation. They demonstrated the concept of impact vibration harvester using a plastic beam with $10 \mathrm{~cm}$ long, $1 \mathrm{~cm}$ wide, $1 \mathrm{~mm}$ thick having $8 \mathrm{~g}$ proof mass as the driving beam and a $27 \mathrm{~mm}$ long, $6.4 \mathrm{~mm}$ wide, $0.51 \mathrm{~mm}$ thick PZT beam as the generating beam. When the driving beam impacts the generating beam, vibration is excited first at the system's coupled vibration frequency and then at the generating beam's higher resonant frequency, producing electrical power. Therefore, the ambient low frequency is up-converted to high resonance frequency by the periodic impact between the driving beam and rigid generating beam.

Meninger et al. [56] studied an energy harvester and obtained $8 \mu \mathrm{W}$ at $2.52 \mathrm{kHz}$ from an in-plane overlap electrostatic generator. Ma et al. [57] proposed an electrostatic generator and achieved $0.065 \mu \mathrm{W}$ from a $4.5 \mathrm{kHz}$ vibration. Kuehne et al. [58] reported a resonant based electrostatic MEMS device with an out-of-plane gap closing mechanism which provided an output power of $4.28 \mu \mathrm{W}$ under vibration with frequency $1 \mathrm{kHz}$ and 
amplitude $1.96 \mathrm{~m} / \mathrm{s}^{2}$, i.e. $0.2 \mathrm{~g}$. Chiu et al. [59] developed an electrostatic MEMS energy harvester using an in-plane gap closing mechanism with a $1 \mathrm{~cm}^{2}$ chip area. An AC output power of $1.2 \mu \mathrm{W}$ with a load of $5 \mathrm{M} \Omega$ was measured at $1.87 \mathrm{kHz}$.

El-Hami et al. [60] studied a vibration-based magnet-coil power generator for embedded remote microsystem structure. They demonstrated that by using high performance $\mathrm{NdFeB}$ magnets and considering a vibration source with a frequency of $320 \mathrm{~Hz}$, maximum output power of $0.53 \mathrm{~mW}$ can be achieved within a volume of $240 \mathrm{~mm}^{3}$. Sari et al. [61] reported a micro-electromagnetic harvester capable of harvesting energy in a wide range of frequencies. They showed that by using several serially connected cantilevers and a central magnet a continuous power of $0.4 \mu \mathrm{W}$ can be generated in a large input frequency band of $800 \mathrm{~Hz}$. However, there are some problems in the fabrication of high performance magnets that can be integrated in magnetic MEMS harvesters. Traditional fabrication processes are not suitable anymore and it requires high processing temperatures which are not compatible with CMOS process. This problem can be solved by using electroplating technology [62] which is suitable for microstructures. On this basis, CoNiMnP permanent magnet arrays can be fabricated for potential application in MEMS sensor and actuators. Han et al. [63] designed a magnetic MEMS energy harvester with the combination of electroplated CoNiMnP permanent micro magnets and microfabricated metal structures.

They showed that the designed harvester with a dimension of $5 \mathrm{~mm} \times 5 \mathrm{~mm} \times 0.53 \mathrm{~mm}$ can harvest $0.03 \mu \mathrm{W} / \mathrm{cm}^{2}$ output power density at $64 \mathrm{~Hz}$. In addition, its small size makes it suitable for batch fabrication through the MEMS process. In general, the electrostatic mechanism has the lowest energy harvesting capabilities amongst the other types of energy harvesters [64]. However electrostatic energy harvesters do have specific advantages and areas of application. For example, they are mainly made of silicon by using semiconductor fabrication technology and this facilitates CMOS integration.

The MEMS vibration-based harvesting device has AC output that needs to be rectified. Almost all the rectifying semiconductor devices consume at least $500 \mathrm{mV}$ as dropped voltage. Hence to overcome this high voltage requirement it is proposed to use the interdigitated electrodes instead of the proposed PZT parallel electrodes [46, 65, 66]. Jeon et al. [46] developed a $\{3-3\}$ mode thin film PZT cantilever device with inter-digitated electrodes that can generate $1.0 \mu \mathrm{W}$ from $10.8 \mathrm{~g}$ vibration at $13.9 \mathrm{kHz}$ resonance frequency. Lee et al. [65], designed and fabricated piezoelectric MEMS micro-generator 
with laminated $\{3-3\}$ mode PZT cantilever and interdigitated electrodes that can generate $0.123 \mu \mathrm{W}$ under $2 \mathrm{~g}$ acceleration amplitude. Similarly Lee et al. [66] developed two piezoelectric MEMS generators with $\{3-1\}$ mode and $\{3-3\}$ mode, having a cantilever made by a silicon micromachining process. The experimental results showed that $\{3-1\}$ mode micro-generator could generate output power of $2.765 \mu \mathrm{W}$ excited at $2.5 \mathrm{~g}$ amplitude and $255.9 \mathrm{~Hz}$ resonance frequency, while the $\{3-3\}$ mode generator could generate an output power of $1.288 \mu \mathrm{W}$ under $2 \mathrm{~g}$ amplitude and $214 \mathrm{~Hz}$.

Most energy harvesters are designed to work at resonance frequency in order to obtain maximum output power, and they are usually manufactured to have resonance frequencies that match the frequencies of excitation. However, in some cases, there is a mismatch between the resonance and excitation frequencies due to manufacturing errors or changes in the working environment. To overcome this problem, harvesters with adjustable natural frequencies [67] and multiple oscillators [68] have been proposed to improve the performance of the harvesters. Furthermore, the use of damping to allow better extraction over a broad frequency band [69] and the use of nonlinear behaviour [70] and magnetic buckling [71] have been exploited to harvest energy efficiently over a wider frequency range. There are different concepts through which the resonance frequency of the harvester can be tuned.

Generally, the resonance frequency can be tuned "actively" or "passively" [72]. For the active mode we need continuous power input to tune the resonance frequency, while in the passive mode, intermittent power is used for tuning process. Resonance tuning methods can be categorized into mechanical, magnetic, and piezoelectric methods. Furthermore, tuning the resonance frequency can be implemented manually or in a selftuning way. Manual tuning is quite complicated to implement during operation. A fine self-tuning implementation is expected not only to cover the targeted frequency range but also to be capable of self-detecting the frequency. Based on elementary of vibration theory, the resonance of a system can be tuned by changing the stiffness or mass. In real life application, it is more practical to change the stiffness rather than the mass of the system. Leland and Wright [73] and $\mathrm{Hu}$ et al. [74] proposed to apply axial preload to alter the stiffness in their energy harvesting devices, thus tuning the resonant frequencies. In Leland and Wright's work, an axial compressive load was applied on a simply supported bimorph energy harvester. In their experimental test on the prototype with a $7.1 \mathrm{~g}$ proof mass, it was determined that before the bimorph failure, a compressive axial preload can 
reduce its resonance frequency by up to $24 \%$. Over the frequency range of $200-250 \mathrm{~Hz}$, this porotype achieved a power output of $300-400 \mathrm{~W}$ under a $1 \mathrm{~g}$ excitation acceleration. $\mathrm{Hu}$ et al. [74] derived the governing equations of a cantilever piezoelectric bimorph with an axial preload and investigated its feasibility and resonance characteristics. The resonance can be adjusted either higher or lower with a tensile or compressive load, respectively. In their model, it was reported that a tensile load of $50 \mathrm{~N}$ increased the resonance from 129.3 to $169.4 \mathrm{~Hz}$ while the same compressive load decreased the resonance from 129.3 to $58.1 \mathrm{~Hz}$.

Marzencki et al. [75] employed mechanical nonlinear strain stiffening to tune the resonance frequency of a MEMS vibration energy harvester. Zhu et al. [76] used permanent magnets to adjust the natural frequency of an electromagnetic microgenerator. They showed that by applying different axial tensile forces to the microgenerator, the natural frequency of the system can be tuned. Challa et al. [77] investigated a vibration energy harvesting device with autonomously tuneable resonance frequency. They used a piezoelectric cantilever beam array with magnets attached to the free ends of cantilever beams to tune the resonance frequency of the system by magnetic force. More recently, Miller et al. [78] proposed a passive self-tuning beam resonator with sliding proof mass along the beam. This model enables the energy harvesting system to adjust the natural frequency of the system and thereby increase the energy harvested over time. Zhang et al. [79] proposed and fabricated an electret-based energy harvester with high normalized power density (NPD, harvested power/volume/acceleration ${ }^{2}$ ) and broad bandwidth. They showed that the proposed model works with a gap-closing scheme packaged at a low pressure, which increases the Q-factor and reduces the air damping. In addition, nonlinear techniques have been investigated to design broadband energy harvesters with no tuning effort required and a better power density. For example, using spring nonlinearity the bandwidth of the harvester can be expanded through several mechanisms such as magnetic levitation, non-linear stiffness and piezoelectric coupling [80]. Nonlinear techniques for broadband the frequency range of energy harvesters focus mostly on the generation of nonlinear force by a Duffing-type oscillator. This type of oscillators include monostable [81], bistable [82], and tristable [83] nonlinear configurations.

Generally, nonlinearity appears much more inherently in monostable cases. Therefore, the total volume is often not that bulky and suitable for integrated processes [84]. 
Consequently, stretching-based monostable nonlinearity has been investigated by numerous researchers for broadband energy harvesting including macro and micro scale types. Leadenham and Erturk [85] reported an M-shaped asymmetric nonlinear oscillator for broadband vibration energy harvesting, which can be announced as yielding broadband behavior under low excitation levels. Gafforelli et al [86] propose a bridge shaped double clamped beam to widen the frequency by a stretching mechanism. Hajati et al [87] reported a mems piezoelectric energy harvester with a doubly clamped beam showing ultra wide-bandwidth due to stretching strain. Marzencki et al [75] reported mems piezoelectric clamped-clamped beam energy harvester device with passive resonance frequency adaption capabilities under high excitation amplitude. Mallick et al [88] described two nonlinear spring architectures with a fixed-fixed configuration for wideband output responses. Liu et al [89] reported a hybrid frequency broadening mechanism combining clamped -clamped beam stretching and different resonant modals.

\subsection{Thesis overview}

Here is a short overview of this thesis.

\section{Chapter 2: Modelling of cantilevered piezoelectric energy harvesters}

In this chapter, the theory of cantilevered piezoelectric energy harvesters for bimorph configuration with series and parallel connections is presented. In addition, the electromechanically coupled circuit equation will be derived based on Gauss's law and the relevant piezoelectric constitutive equation.

Chapter 3: Modelling and design of MEMS piezoelectric energy harvester with adjustable resonance frequency

This chapter presents a comprehensive study and a framework for the modelling and design of a MEMS piezoelectric harvester which employs an electrostatic device to adjust its resonance frequency. The main purpose of the proposed model is to control the resonance frequency of the MEMS piezoelectric harvesters with the DC voltage applied to the electrostatic system to maximize the harvested power. 
Chapter 4: Effects of manufacturing uncertainties in MEMS Energy Harvesters

In this chapter, the performance of MEMS piezoelectric harvesters in the presence of manufacturing uncertainties is investigated. Based on the experimentally measured statistical properties available in the literature, manufacturing uncertainty in MEMS harvesters results in a lower output power. In order to improve the performance of the harvester two electrostatic tuning mechanisms will be used.

Chapter 5: Experimental studies of an energy harvester with adjustable resonance frequency in macroscale

Chapter 5 proposes an experimental set up to show the electrostatic softening mechanis $\mathrm{m}$ by using electromagnets in macro-scales. Based on this model, applying voltage to the electromagnets will tune the resonance frequency of the harvester.

Chapter 6: Conclusion and future work

In the final chapter of this thesis the results are summarized and an outlook for suggested future work regarding energy harvesters is given.

\subsection{Journal and conference papers based on this thesis}

The results of the current thesis have been published in the following papers

[1]. Hadi Madinei, Hamed Haddad Khodaparast, Sondipon Adhikari, Michael I. Friswell, Meghdad Fazeli, "Adaptive tuned piezoelectric MEMS vibration energy harvester using an electrostatic device" The European Physical Journal, Special issue: Nonlinear and Multiscale Dynamics of Smart Materials in Energy Harvesting, Vol. 224, November 2016, pp. 2703-2717. 
[2]. Hadi Madinei, Hamed Haddad Khodaparast, Sondipon Adhikari, Michael I Friswell, "A Hybrid Piezoelectric and Electrostatic Vibration Energy Harvester", Shock \& Vibration, Aircraft/Aerospace, Energy Harvesting, Acoustics \& Optics, Vol. 9, 2016, pp. 189-195.

[3]. Hamed Haddad Khodaparast, Hadi Madinei, Michael I Friswell, Sondipon Adhikari, "Vibration suppression in MEMS devices using electrostatic forces", SPIE Smart Structures and Materials+ Nondestructive Evaluation and Health Monitoring, April 2016, pp. 979917-979917-9.

[4]. Hadi Madinei, Hamed Haddad Khodaparast, Sondipon Adhikari, Michael I Friswell, "Design of MEMS piezoelectric harvesters with electrostatically adjustable resonance frequency”, Mechanical Systems and Signal Processing, Vol. 81, December 2016, pp. 360-374.

[5]. Hadi Madinei, Hamed Haddad Khodaparast, Sondipon Adhikari, Michael I Friswell, "Improving performance of MEMS piezoelectric harvesters in the presence of manufacturing uncertainties", International Conference on Uncertainty in Structural Dynamics (USD2016), At Leuven, Belgium.

[6]. Hamed Haddad Khodaparast, Hadi Madinei, Michael I Friswell, Sondipon Adhikari, Simon Coggon, Jonathan E Cooper, "An extended harmonic balance method based on incremental nonlinear control parameters", Mechanical Systems and Signal Processing, Vol. 85, February 2017, pp. 716-729.

[7]. Hadi Madinei, Hamed Haddad Khodaparast, Michael I Friswell, Sondipon Adhikari, Alexander David Shaw, "Nonlinear MEMS Piezoelectric Harvesters in the presence of geometric and structural variabilities", Procedia Engineering, Volume 199, 2017, Pages 3456-3461.

[8]. Hadi Madinei, Hamed Haddad Khodaparast, Michael I Friswell, Sondipon Adhikari, "Minimising the effects of manufacturing uncertainties in MEMS Energy Harvesters", International journal of energy, February 2018 (Accepted).

[9]. Hadi Madinei, Hamed Haddad Khodaparast, Alexander D. Shaw, Michael I Friswell, "Broadening the frequency band of a vibration-based energy harvester 
using electromagnetic devices", Submitted to International Conference on Vibration Engineering and Technology of Machinery, January 2018 (Accepted for oral presentation and publication).

[10]. Hadi Madinei, Hamed Haddad Khodaparast, Javad Taghipour, Michael I Friswell, "Identification of electromagnetic nonlinear restoring force in a broadband resonator", (In preparation for submission to a journal). 


\section{Chapter 2: Modelling of cantilevered piezoelectric energy harvesters}

In this chapter, an analytical model for a cantilevered vibration-based piezoelectric energy harvester is presented. The model proposed by Erturk and Inman [90] is used as a basis for the linear piezoelectric energy harvester model. After deriving the mechanical and electrical equations representing the dynamic of the harvester, a steady-state solution is presented to study the behaviour of the harvester.

\subsection{Mechanical equation of motion}

In this section, an expression for the coupled beam equation of bimorph piezoelectric harvester is derived. Figure 2.1 shows a bimorph piezoelectric harvester in two configurations based on the connection between piezoelectric layers. By considering the base excitation, the partial differential equation of motion can be written as [90]

$$
-\frac{\partial^{2} M_{b}}{\partial x^{2}}+m \frac{\partial^{2} w_{r e l}}{\partial t^{2}}+c_{a} \frac{\partial w_{r e l}}{\partial t}=-\left(m+M_{t} \delta(x-L)\right) \frac{\partial^{2} w_{b}}{\partial t^{2}}
$$

where $w_{r e l}$ is the vertical displacement of the beam relative to its base, $m$ is the mass per unit length of the beam, $M_{t}$ is the tip mass, $\delta(x)$ is the Dirac delta function, $w_{b}$ is the base displacement and $c_{a}$ is the viscous air damping coefficient; $M_{b}$ refer to the internal bending moment and represented as

$$
M_{b}=b\left(\int_{-h_{\tilde{p}}-\frac{h_{\tilde{S}}}{2}}^{-\frac{h_{\tilde{S}}}{2}} T_{\tilde{p}} z d z+\int_{-\frac{h_{\tilde{S}}}{2}}^{\frac{h_{\tilde{S}}}{2}} T_{\tilde{S}} z d z+\int_{\frac{h_{\tilde{S}}}{2}}^{\frac{h_{\tilde{S}}}{2}+h_{\tilde{p}}} T_{\tilde{p}} z d z\right)
$$

where $h_{\tilde{S}}$ is the thickness of the beam, $h_{\tilde{p}}$ is the thickness of each piezoceramic layer, and $\mathrm{b}$ is the width of the cantilever beam and each piezoceramic layer and is supposed to be constant along the beam length. 


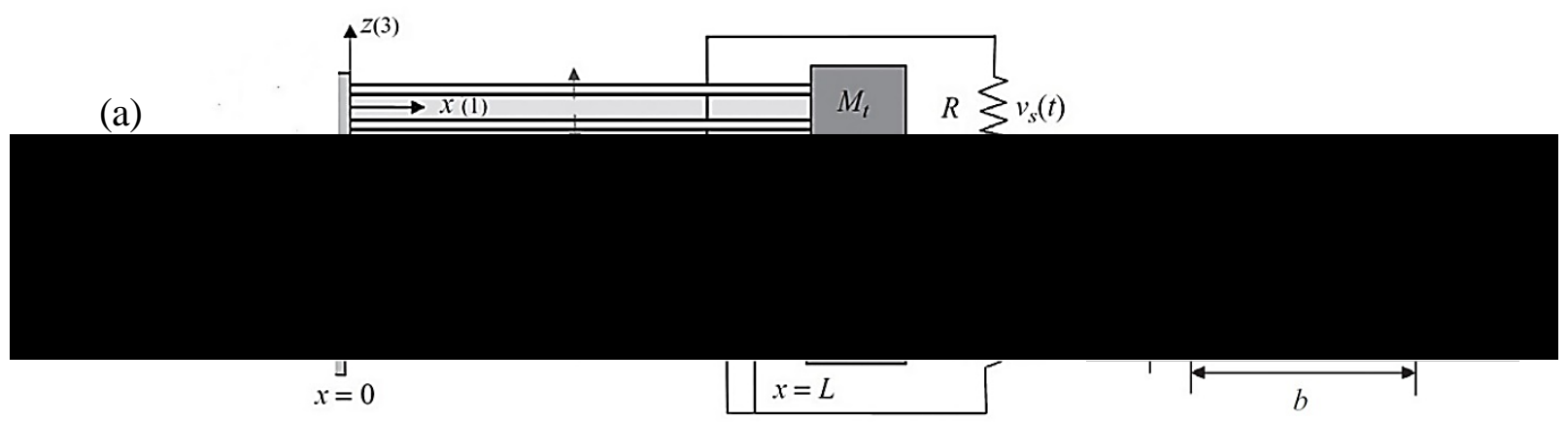

$\square$ Piezoceramic $\square$ Substructure — Electrodes $\uparrow$ Poling direction

(c)

Figure 2.1 Bimorph piezoelectric energy harvester configurations, (a) series and (b) parallel connection of piezoceramic layers and (c) their cross-sectional view [90]

The subscripts $\tilde{S}$ and $\tilde{p}$ stand for the beam and piezoelectric material. In addition, $T_{\tilde{S}}$ and $T_{\tilde{p}}$ are the stress components in the beam and piezoceramic. These stress components are given in the following equations

$$
T_{\tilde{s}}=E_{\tilde{s}} S_{\tilde{s}}, \quad T_{\tilde{p}}=\bar{c}_{11}^{E} S_{\tilde{p}}-\bar{c}_{11}^{E} d_{31} E_{3}
$$

where $E_{\tilde{S}}$ is the elastic modulus of the beam, $\bar{c}_{11}^{E}$ is the elastic modulus of the piezoceramic layers at a constant electric field, $E_{3}$ is the electric field component in the poling direction (i.e. $\mathrm{z}$ direction), $d_{31}$ is the piezoelectric stress constant (unit F. $\mathrm{m}^{-1}$ ) and $S$ is the axial strain expressed as

$$
S(x, z, t)=-z \frac{\partial^{2} w_{r e l}}{\partial x^{2}}
$$

In addition, considering the plane-stress assumption for a transversely isotropic thin piezoceramic beam, the elastic modulus component of the piezoceramic can be written as

$$
\bar{c}_{11}^{E}=\frac{1}{s_{11}^{E}}
$$

where $S_{11}^{E}$ is the elastic compliance (i.e. the strain produced in a piezoelectric material per unit of stress applied) at a constant electric field. Considering the same assumption, $d_{31}$ can be given by 


$$
d_{31}=\bar{e}_{31} s_{11}^{E}
$$

where $\bar{e}_{31}$ is the effective piezoelectric stress-constant. Using equations (2.5) and (2.6), the stress component for piezoceramic can be written as

$$
T_{\tilde{p}}=\bar{c}_{11}^{E} S_{\tilde{p}}-\bar{e}_{31} E_{3}
$$

It is noticeable that Equation (2.7), is valid only for small amplitude vibration. The electric field $\left(E_{3}\right)$ depends on the connections between the piezoceramic layers and is written in terms of the corresponding voltage for series and parallel connections. The voltage across the electrodes of each piezoceramic layer in the series connection is $v_{s}(t) / 2$ and, as expected, in the parallel connection is $v_{p}(t)$. Due to the opposite polling in the series connection, $\bar{e}_{31}$ has the opposite sign for top and bottom electrodes. Therefore, the electrical field for each bimorph configuration can be written as

$$
\begin{aligned}
E_{3}^{s}(t) & =-\frac{v_{s}(t)}{2 h_{\tilde{p}}} \\
E_{3}^{p}(t) & =\left\{\begin{array}{l}
-\frac{v_{p}(t)}{h_{\tilde{p}}}: \text { Top layer } \\
\frac{v_{p}(t)}{h_{\tilde{p}}}: \text { Bottom layer }
\end{array}\right.
\end{aligned}
$$

It is noteworthy that for the electrode configuration shown in Figure 2.1, $E_{1}$ and $E_{2}$ are zero. The subscripts $s$ and $p$ stand for the series and parallel connections. For both configurations, the piezoelectric coupling term in equation (2.2) is only a time-dependent function, therefore due to the spatial differentiation in equation (2.1), it will be eliminated after substituting equation (2.2) into equation (2.1). Consequently, it must be multiplied by Heaviside function. Using equations (2.3), (2.4) and (2.7), the internal bending moment for series and parallel connections are given by

$$
\begin{aligned}
& M_{b}^{s}=-E I \frac{\partial^{2} w_{r e l}^{s}}{\partial x^{2}}+\vartheta_{s} v_{s}(t)[H(x)-H(x-L)] \\
& M_{b}^{p}=-E I \frac{\partial^{2} w_{r e l}^{p}}{\partial x^{2}}+\vartheta_{p} v_{p}(t)[H(x)-H(x-L)]
\end{aligned}
$$

where $E I$ is the bending stiffness term, $\vartheta_{s}$ and $\vartheta_{p}$ are the backward coupling terms for series and parallel connections. These terms can be expressed as [90] 


$$
\begin{aligned}
& E I=\frac{2 b}{3}\left(Y_{\tilde{s}} \frac{h_{\tilde{s}}^{3}}{8}+c_{11}^{E}\left(\left(h_{\tilde{p}}+\frac{h_{\tilde{s}}}{2}\right)^{3}-\frac{h_{\tilde{S}}^{3}}{8}\right)\right) \\
& \vartheta_{s}=\frac{\bar{e}_{31} b}{2 h_{\tilde{p}}}\left(\left(h_{\tilde{p}}+\frac{h_{\tilde{s}}}{2}\right)^{2}-\frac{h_{\tilde{s}}^{2}}{4}\right), \quad \vartheta_{p}=2 \vartheta_{s}=\frac{\bar{e}_{31} b}{h_{\tilde{p}}}\left(\left(h_{\tilde{p}}+\frac{h_{\tilde{s}}}{2}\right)^{2}-\frac{h_{\tilde{s}}^{2}}{4}\right)
\end{aligned}
$$

By substituting equation (2.9) in equation (2.1), the coupled beam equation for the parallel connection can be written as

$$
\begin{aligned}
& E I \frac{\partial^{4} w_{r e l}^{p}}{\partial x^{4}}+m \frac{\partial^{2} w_{r e l}^{p}}{\partial t^{2}}+c_{a} \frac{\partial w_{r e l}^{p}}{\partial t}-\vartheta_{p} v_{p}(t)\left(\frac{d \delta(x)}{d x}-\frac{d \delta(x-L)}{d x}\right) \\
& =-\left(m+M_{t} \delta(x-L)\right) \frac{\partial^{2} w_{b}}{\partial t^{2}}
\end{aligned}
$$

Similarly, the coupled beam equation can be derived for series connection. In equation (2.11), the mass per unit length is

$$
m=b\left(\rho_{\tilde{s}} h_{\tilde{s}}+2 \rho_{\tilde{p}} h_{\tilde{p}}\right)
$$

where $\rho_{\tilde{p}}$ and $\rho_{\tilde{s}}$ are the densities of the piezoceramic and the beam, respectively.

\subsection{Coupled electrical equation}

In order to obtain the coupled electrical equation for series and parallel connections, one should first study the electrical equation of a single piezoceramic layer under base excitation. To this end, one of the piezoceramic layers in Figure 2.1 is connected to a load resistor. By considering the axial mechanical strain due to the bending vibration, the piezoelectric constitutive equation can be expressed as [90]

$$
D_{3}=\bar{e}_{31} S_{\tilde{p}}+\bar{\varepsilon}_{33}^{\tilde{s}} E_{3}
$$

where $D_{3}$ is the electric displacement component and $\bar{\varepsilon}_{33}^{\tilde{s}}$ is the permittivity component at constant strain with the plane stress assumption for the beam. Based on Gauss's law, the generated charge, $q(t)$ can be achieved by integrating electric displacement over the electrode area, $A_{e}$ 


$$
q(t)=\iint D_{3} d A_{e}
$$

Since the external circuit admittance across the electrodes is $1 / R$, therefore the output electrical current can be expressed as

$$
i(t)=\frac{d}{d t}\left(\iint D_{3} d A_{e}\right)=\frac{v(t)}{R}
$$

After using equation (2.4) to express the average bending strain in the piezoceramic in form of the curvature and considering the electrical field as $-v_{p}(t) / h_{\tilde{p}}$, equation $(2.13)$ can be used in equation (2.15) to obtain

$$
-C_{\tilde{p}} \frac{d v(t)}{d t}-\bar{e}_{31} b h_{\tilde{p} c} \int_{0}^{L} \frac{\partial^{3} w_{r e l}}{\partial x^{2} \partial t} d x=\frac{v(t)}{R}
$$

where $h_{\tilde{p} c}$ is the distance between the center of each piezoceramic layer and the neutral axis and $C_{\tilde{p}}$ refers to the piezoelectric internal capacitance

$$
C_{\tilde{p}}=\frac{\bar{\varepsilon}_{33}^{S} b L}{h_{\tilde{p}}}
$$

According to equation (2.16), a simple RC circuit can be used to model the energy harvester. Therefore, as shown in Figure 2.2 each piezoceramic layer can be shown as a dependent current source in parallel with its internal capacitance.

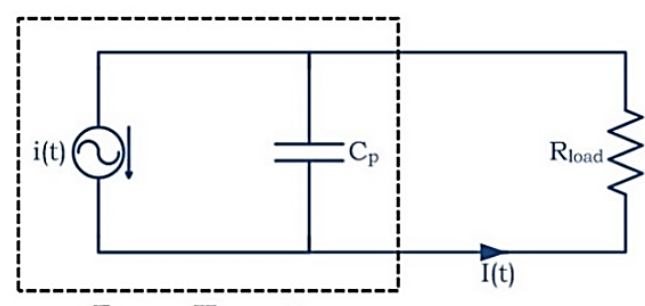

Energy Harvester

Figure 2.2 Electrical representation of a piezoceramic layer connected to a resistor. 


\subsubsection{Coupled electrical equation for series and parallel connections}

Based on the concepts described in Section 2.1, this section aims to study the electrical equation for series and parallel connections. Considering the electrical representation of each piezoceramic layer, Figure 2.3 shows the series connection of the piezoceramic layer in bimorph configuration.

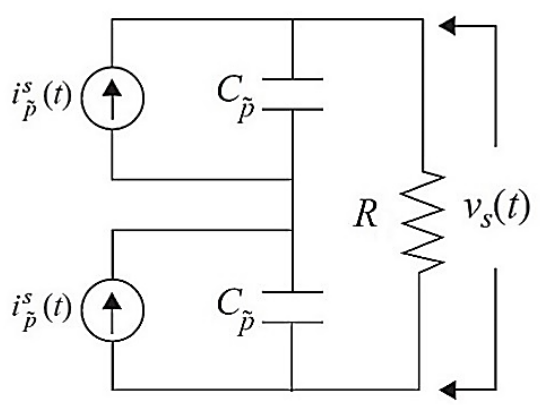

Figure 2.3 Electrical circuit representing the series connection of the piezoceramic layers.

Using Kirchhoff's laws for the circuit shown in Figure 2.3 gives

$$
\frac{C_{\tilde{p}}}{2} \frac{d v_{s}(t)}{d t}+\frac{v_{s}(t)}{R}+i_{\tilde{p}}^{S}(t)=0
$$

where

$$
C_{\tilde{p}}=\frac{\bar{\varepsilon}_{33}^{S} b L}{h_{\tilde{p}}}, \quad i_{\tilde{p}}^{S}(t)=\frac{\bar{e}_{31} b\left(h_{\tilde{p}}+h_{\tilde{s}}\right)}{2} \int_{0}^{L} \frac{\partial^{3} w_{r e l}^{S}}{\partial x^{2} \partial t} d x
$$

similarly, the electrical circuit of the parallel connection of the piezoceramic layer can be represented by Figure 2.4.

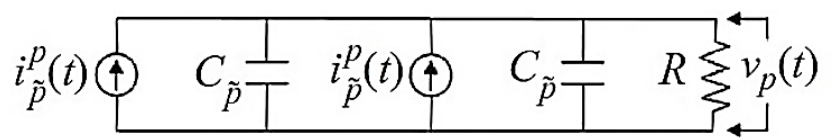

Figure 2.4 Electrical circuit representing the parallel connection of the piezoceramic layers. 
By applying Kirchhoff's laws, the governing electrical circuit equation can be written as

$$
C_{\tilde{p}} \frac{d v_{p}(t)}{d t}+\frac{v_{p}(t)}{2 R}+i_{\tilde{p}}^{p}(t)=0
$$

where

$$
C_{\tilde{p}}=\frac{\bar{\varepsilon}_{33}^{s} b L}{h_{\tilde{p}}}, \quad i_{\tilde{p}}^{p}(t)=\frac{\bar{e}_{31} b\left(h_{\tilde{p}}+h_{\tilde{s}}\right)}{2} \int_{0}^{L} \frac{\partial^{3} w_{r e l}^{p}(x, t)}{\partial x^{2} \partial t} d x
$$

Depending on the voltage or current requirements, the piezoceramic layers can be connected in series or parallel configurations. Using piezoceramic layers in series connection produces a larger voltage output, whereas in parallel connection a larger current output can be obtained.

\subsection{Voltage and vibration response at steady state}

In this section, the Galerkin decomposition method is used to eliminate spatial dependence. To this end, the vibration response relative to the base of the beam can be represented as a series expansion in terms of the eigenfunctions of the beam, i.e.

$$
w_{r e l}^{p}(x, t)=\sum_{i=1}^{N} U_{i}(t) \varphi_{i}(x)
$$

where $\varphi_{i}(x)$ is the ith linear undamped mode shape of the straight beam and $U_{i}(t)$ is the ith generalized coordinate. Considering the parallel configuration and $z_{0} e^{j \Omega \mathrm{t}}$ as a base excitation equations (2.11) and (2.20) can be converted into a system of differential equations using the Galerkin decomposition method. Substituting equation (2.12) into equations (2.11) and (2.20) and multiplying by $\varphi_{i}(x)$ as a weight function in Galerkin method and integrating the outcome from $x=0$ to 1 , a system of differential equations can be obtained. Assuming a single-mode approximation yields the following equations

$$
\begin{gathered}
M \ddot{U}(t)+C \dot{U}(t)+K_{m} U(t)-\theta_{p} v_{p}(t)=F_{b} e^{j \Omega t} \\
C_{\tilde{p}} \frac{d v_{p}(t)}{d t}+\frac{v_{p}(t)}{2 R}+\frac{\theta_{p}}{2} \dot{U}(t)=0
\end{gathered}
$$


where

$$
\begin{aligned}
& M=m \int_{0}^{L} \varphi^{2}(x) d x, C=c_{a} \int_{0}^{L} \varphi^{2}(x) d x, K_{m}=E I \int_{0}^{L} \varphi(x) \varphi^{I V}(x) d x \\
& \theta_{p}=\vartheta_{p} \frac{d \varphi(L)}{d x}, F_{b}=z_{0} \Omega^{2}\left(m \int_{0}^{L} \varphi(x) d x+M_{t} \int_{0}^{L} \varphi(x) \delta(x-L) d x\right)
\end{aligned}
$$

Since the beam is excited at frequency $\Omega$, the steady-state response of the beam and the steady-state voltage across the load resistance are considered as following

$$
\begin{gathered}
U(t)=A e^{j \Omega t} \\
v_{p}(t)=B e^{j \Omega t}
\end{gathered}
$$

where A and B are complex values. Substituting equations (2.26) and (2.27) into equations (2.23) and (2.24), yields the following two equations for $\mathrm{A}$ and $\mathrm{B}$

$$
\begin{gathered}
\left(K_{m}-M \Omega^{2}+j C \Omega\right) A-\theta_{p} B=F_{b} \\
\left(2 C_{\tilde{p}} j \Omega+\frac{1}{R}\right) B+\theta_{p} A j \Omega=0
\end{gathered}
$$

By solving above set of linear equations, the steady-state response of the beam and steadystate voltage response can be expressed as

$$
\begin{gathered}
U(t)=\frac{\left(2 C_{\tilde{p}} j \Omega+\frac{1}{R}\right) F_{b}}{j \theta_{p}^{2} \Omega+\left(K_{m}-M \Omega^{2}+j C \Omega\right)\left(2 C_{\tilde{p}} j \Omega+\frac{1}{R}\right)} e^{j \Omega \mathrm{t}} \\
v_{p}(t)=-\frac{j F_{b} \theta_{p} \Omega}{j \theta_{p}^{2} \Omega+\left(K_{m}-M \Omega^{2}+j C \Omega\right)\left(2 C_{\tilde{p}} j \Omega+\frac{1}{R}\right)} e^{j \Omega \mathrm{t}}
\end{gathered}
$$

Using equation (2.22) and considering a single-mode approximation, the transverse relative displacement response at point $x$ on the beam can be expressed as

$$
w_{r e l}^{p}(x, t)=\frac{\left(2 C_{\tilde{p}} j \Omega+\frac{1}{R}\right) F_{b}}{j \theta_{p}^{2} \Omega+\left(K_{m}-M \Omega^{2}+j C \Omega\right)\left(2 C_{\tilde{p}} j \Omega+\frac{1}{R}\right)} \varphi(x) e^{j \Omega \mathrm{t}}
$$

Similarly, by following the same procedure for series configuration the following equation can be obtained 


$$
\begin{gathered}
w_{r e l}^{S}(x, t)=\frac{\left(0.5 C_{\tilde{p}} j \Omega+\frac{1}{R}\right) F_{b}}{j \theta_{s}^{2} \Omega+\left(K_{m}-M \Omega^{2}+j C \Omega\right)\left(0.5 C_{\tilde{p}} j \Omega+\frac{1}{R}\right)} \varphi(x) e^{j \Omega \mathrm{t}} \\
v_{s}(t)=-\frac{j F_{b} \theta_{s} \Omega}{j \theta_{s}^{2} \Omega+\left(K_{m}-M \Omega^{2}+j C \Omega\right)\left(0.5 C_{\tilde{p}} j \Omega+\frac{1}{R}\right)} e^{j \Omega \mathrm{t}}
\end{gathered}
$$

Note that if one is interested in the total displacement of the beam, it is the summation of the base displacement and the transversal relative displacement. Furthermore, in equations (2.31), (2.32), (2.33) and (2.34) by taking the limit as $R \rightarrow \infty$ and $R \rightarrow 0$, the output voltage and deflection of the beam converge to constant values [90]. The average power harvested between time $t_{1}$ and $t_{2}$ for parallel configuration is calculated by integrating the temporal response as

$$
P_{\text {ave }}=\frac{1}{t_{2}-t_{1}} \int_{t_{1}}^{t_{2}} \frac{\left\{v_{p}(t)\right\}^{2}}{R} d t
$$

and peak power from the piezoelectric layers can be expressed as

$$
P_{\text {max }}=\frac{\left|\left\{v_{p}(t)\right\}_{\max }\right|^{2}}{R}
$$

\subsection{Theoretical case study}

In this section, a MEMS bimorph piezoelectric harvester is considered in parallel configuration to demonstrate the analysis presented in previous sections. The geometric properties of the harvester are given in Table 2.1.

Shape functions, which satisfy the boundary conditions of the clamped-free microbeam (with tip mass $M_{t}$ ), are considered of the form [90]

$$
\varphi_{i}(x)=A_{r}\left[\cos \lambda_{i} \frac{x}{L}-\cosh \lambda_{i} \frac{x}{L}+\varsigma_{r}\left(\sin \lambda_{i} \frac{x}{L}-\sinh \lambda_{i} \frac{x}{L}\right)\right]
$$

where

$$
\varsigma_{r}=\frac{\sin \lambda_{i}-\sinh \lambda_{i}+\lambda_{i} \frac{M_{t}}{m L}\left(\cos \lambda_{i}-\cosh \lambda_{i}\right)}{\cos \lambda_{i}+\cosh \lambda_{i}-\lambda_{i} \frac{M_{t}}{m L}\left(\sin \lambda_{i}-\sinh \lambda_{i}\right)}
$$


$A_{r}$ is the modal amplitude constant and the eigenvalues of the system $\left(\lambda_{i}\right.$ for mode $\left.i\right)$ are obtained from

$$
\begin{gathered}
1+\cos \lambda_{i} \cosh \lambda_{i}+\lambda_{i} \frac{M_{t}}{m L}\left(\cos \lambda_{i} \sinh \lambda_{i}-\sin \lambda_{i} \cosh \lambda_{i}\right)- \\
\frac{\lambda_{i}^{3} I_{t}}{m L^{3}}\left(\cosh \lambda_{i} \sin \lambda_{i}+\sinh \lambda_{i} \cos \lambda_{i}\right)+ \\
\frac{\lambda_{i}^{4} M_{t} I_{t}}{m L^{3}}\left(1-\cos \lambda_{i} \cosh \lambda_{i}\right)=0
\end{gathered}
$$

Table 2.1 Geometrical and material properties of the MEMS harvester

\begin{tabular}{ll}
\hline Design Variable & Value \\
\hline Length $(L)$ & $3000 \mu \mathrm{m}$ \\
Width $(b)$ & $1000 \mu \mathrm{m}$ \\
Thickness $\left(h_{\tilde{s}}\right)$ & $4 \mu \mathrm{m}$ \\
Thickness $\left(h_{\tilde{p}}\right)$ & $2 \mu \mathrm{m}$ \\
Young's modulus $\left(E_{\tilde{S}}\right)$ & $169.6 \mathrm{GPa}$ \\
Young's modulus $\left(c_{11}^{E}\right)$ & $65 \mathrm{GPa}$ \\
Viscous air damping coefficient $\left(c_{a}\right)$ & $0.0002 \mathrm{~N} . \mathrm{s} / \mathrm{m}$ \\
Poisson's ratio $(v)$ & 0.06 \\
Density of Si beam $\left(\rho_{\tilde{s}}\right)$ & $2330 \mathrm{~kg} / \mathrm{m}^{3}$ \\
Density of PZT $\left(\rho_{\tilde{p}}\right)$ & $7800 \mathrm{~kg} / \mathrm{m}^{3}$ \\
Equivalent piezoelectric coefficient $\left(\bar{e}_{31}\right)$ & $-11.18 \mathrm{Cm}{ }^{-2}$ \\
Permittivity component $\left(\bar{\varepsilon}_{33}^{s}\right)$ & $13.48 \mathrm{nF} / \mathrm{m}$ \\
Tip mass $\left(M_{t}\right)$ & $9.724 \times 10^{-8} \mathrm{~kg}$ \\
Length of the tip mass $\left(L_{m}\right)$ & $20 \mu \mathrm{m}$ \\
Thickness of the tip mass $\left(h_{m}\right)$ & $10 \mu \mathrm{m}$ \\
\hline
\end{tabular}

Depending on the load resistance connected to the piezoelectric layers, the system can be in a short-circuit or an open-circuit condition. Considering the first vibration mode of this particular harvester, the lowest resistance is very close to the short-circuit conditions, whereas when using the largest load resistance, the system operates in open-circuit conditions. As the load resistance is increased from the short-circuit to open-circuit conditions, the voltage output at every frequency increases monotonically. On the other hand, in the exact short-circuit condtion $(R=0)$ the output voltage is zero and by increasing the load resistance to the largest load resistance the output voltage converges to its maximum value. In addition, as shown in Figure 2.5, by considering $0.3 \mu \mathrm{m}$ as the amplitude of base excitation and increasing the load resistance from $100 \Omega$ to $100 \mathrm{M} \Omega$ the resonance frequency of the harvester changes from short-circuit resonance frequency 
$\left(\omega_{r}^{s c}\right.$ for $\left.R \rightarrow 0\right)$ to open-circuit resonance frequency $\left(\omega_{r}^{o c}\right.$ for $\left.R \rightarrow \infty\right)$. Therefore, depending on the load resistance the resonance frequency of the harvester can take a value only between $272.8 \mathrm{~Hz}$ and $312.6 \mathrm{~Hz}$.

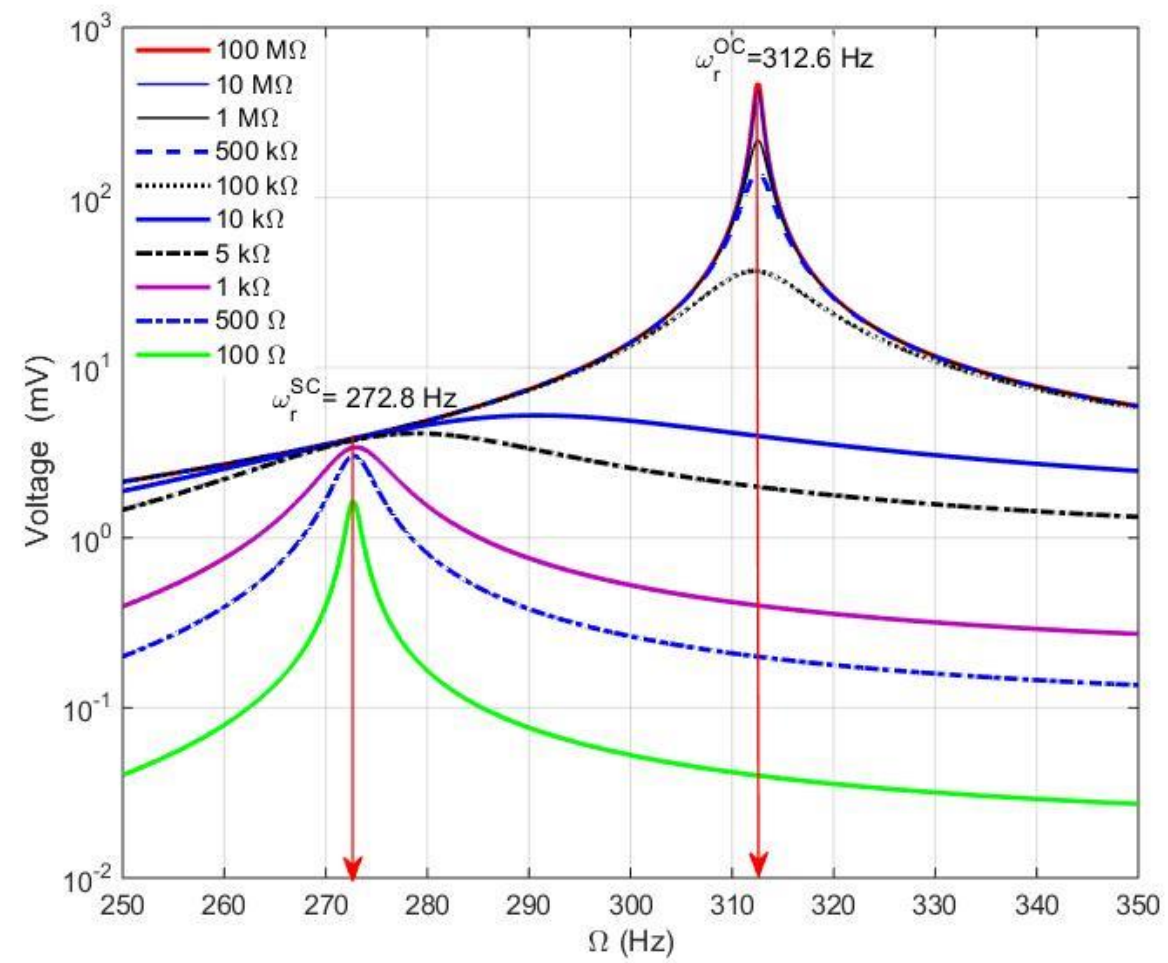

Figure 2.5 Output voltage of the harvester for a broad range of load resistance

It can be seen that the damping caused by power dissipation in the resistor due to Joule heating is more complicated than viscous damping (although it has been oversimplified by several researchers in recent years [91, 92]. Firstly, unlike the case with viscous damping, increasing load resistance shifts the resonance frequency to the right in the FRF. Secondly, by further increasing the load resistance, although the amplitude of vibration decreases in the short-circuit condition (original resonance frequency), it amplifies considerably at the open-circuit resonance frequency (See Figure 2.6).

Changing the electrical boundary conditions shifts the resonance frequency of the harvester. By increasing the load resistance to the upper extremum $(R \rightarrow \infty)$, the elastic modulus of the piezoelectric patch increases from the constant electric field value to the constant electrical displacement value and there is no overall energy dissipation. The 
power dissipation in the mechanical domain is only valid for nonzero and finite values of load resistance, which associated with power generation in the electrical domain.

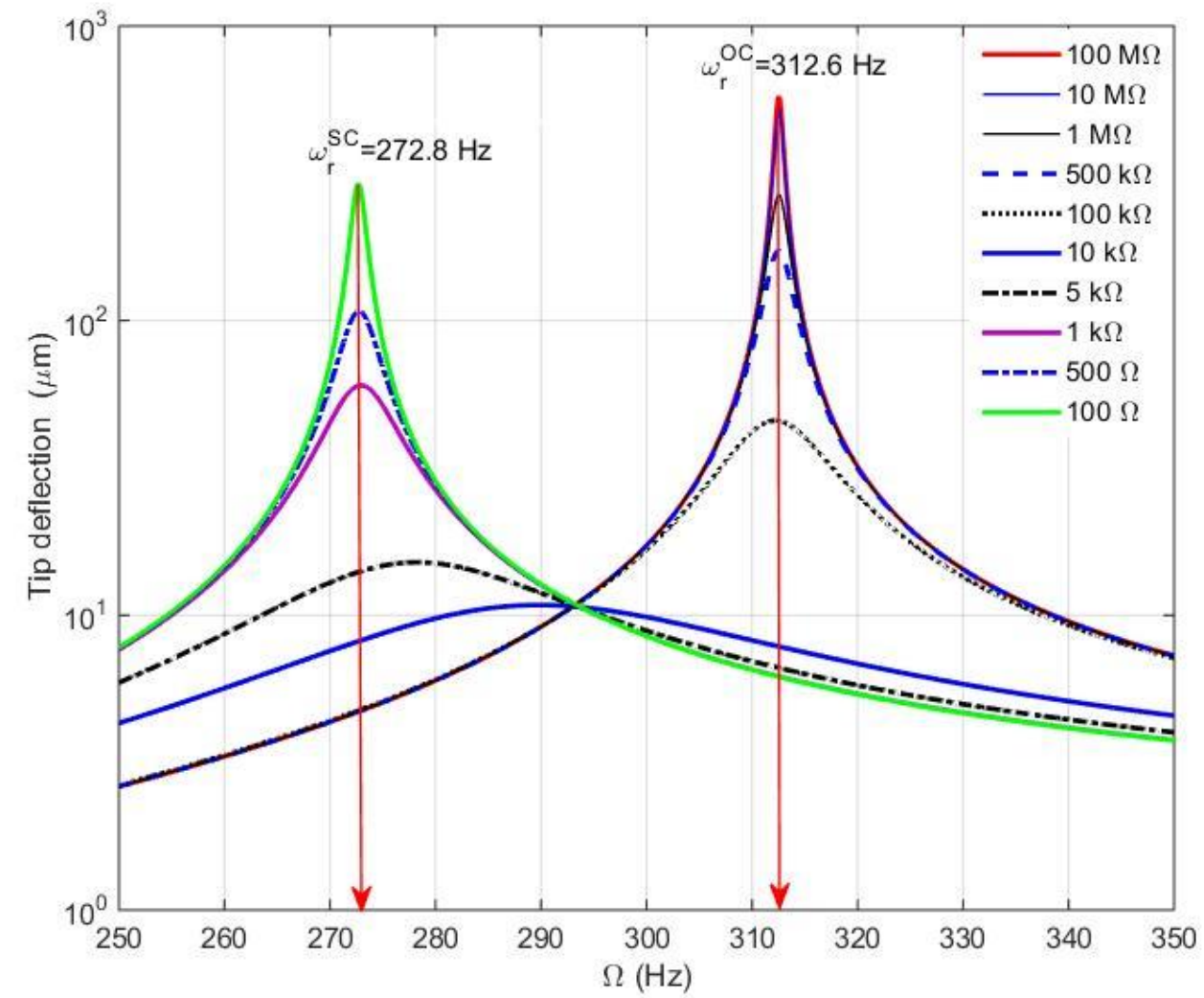

Figure 2.6 Output voltage of the harvester for a broad range of load resistance

Therefore, in the presence of electrical load resistance, the resonance frequency shift cannot be presented by a real-value viscous damping ratio or loss factor [90]. Generally, the resonance frequency shift from short-circuit condition to open-circuit condition is directly proportional to the square of the electromechanical coupling term and inversely proportional to the equivalent capacitance and square of the undamped resonance frequency. In addition, it is also affected by the modal mechanical damping ratio and the effect of electromechanical coupling can be counteracted by the modal mechanical damping ratio. Physically, using very large mechanical losses and/or small electromechanical coupling, the short-circuit resonance frequency can be equal to the open-circuit resonance frequency [90].

To validate the results presented in this chapter, a bimorph cantilever beam has been considered based on Alper Erturk and Daniel J. Inman's book (See Table 2.2). As shown in Figure 2.7, by considering $1 \mathrm{~mm}$ as the amplitude of base excitation and changing the 
load resistance from $100 \Omega$ to $100 \mathrm{M} \Omega$, the resonance frequency of the harvester changes from $185.1 \mathrm{~Hz}$ to $191.1 \mathrm{~Hz}$, which is the same as the results in the book.

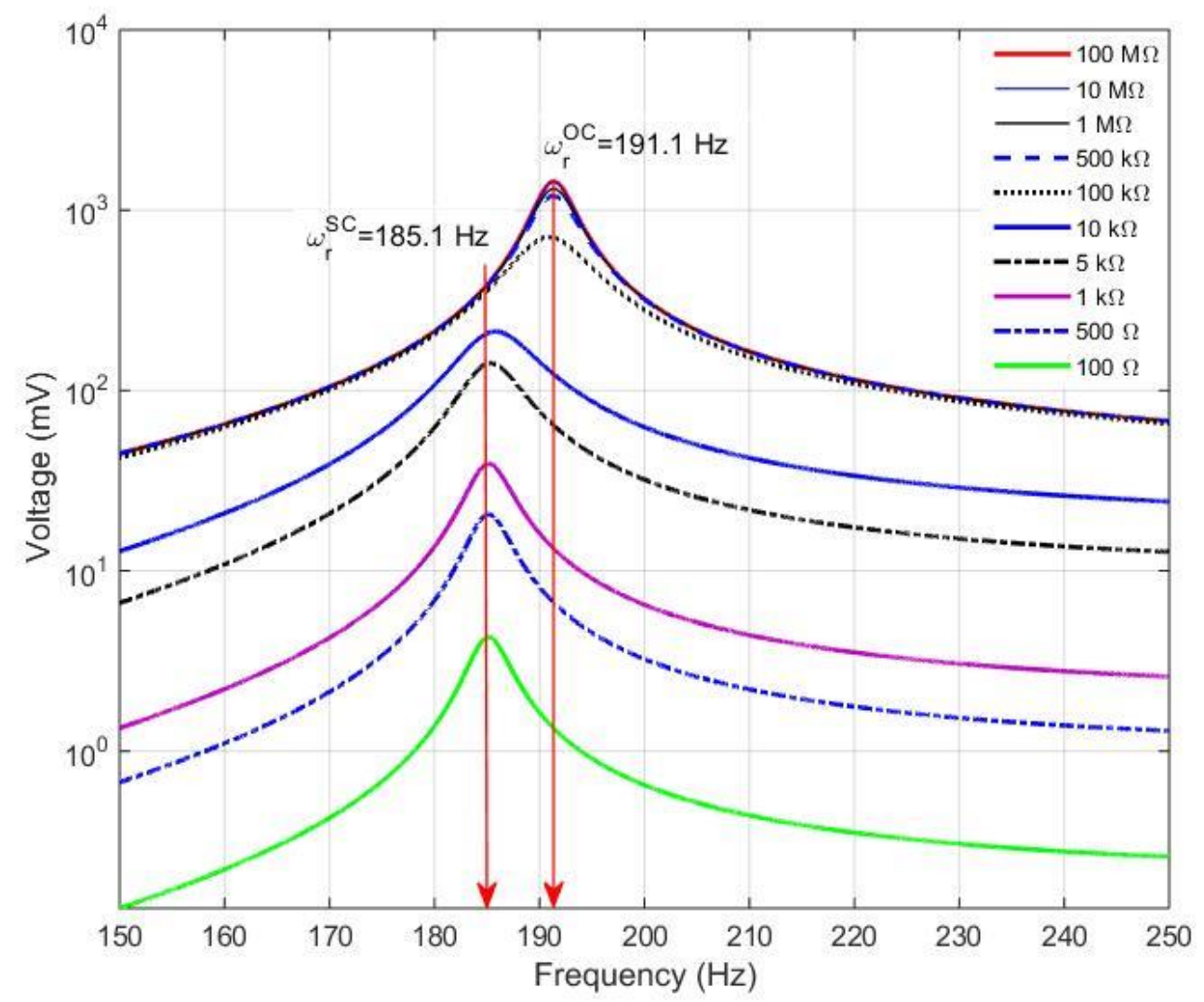

Figure 2.7 Output voltage of the harvester for a broad range of load resistance

Table 2.2 Geometrical and material properties of the harvester [90]

\begin{tabular}{ll}
\hline Design Variable & Value \\
\hline Length $(L)$ & $30 \mathrm{~mm}$ \\
Width $(a)$ & $5 \mathrm{~mm}$ \\
Thickness $\left(h_{\tilde{s}}\right)$ & $0.05 \mu \mathrm{m}$ \\
Thickness $\left(h_{\tilde{p}}\right)$ & $0.15 \mu \mathrm{m}$ \\
Young's modulus $\left(E_{\tilde{\tilde{s}}}\right)$ & $70 \mathrm{GPa}$ \\
Young's modulus $\left(c_{11}^{E}\right)$ & $61 \mathrm{GPa}$ \\
Damping ratio $(\zeta)$ & 0.01 \\
Density of beam $\left(\rho_{\tilde{s}}\right)$ & $2700 \mathrm{~kg} / \mathrm{m}^{3}$ \\
Density of PZT $\left(\rho_{\tilde{p}}\right)$ & $7750 \mathrm{~kg} / \mathrm{m}^{3}$ \\
Equivalent piezoelectric coefficient $\left(\bar{e}_{31}\right)$ & $-10.4 \mathrm{Cm}-2$ \\
Permittivity component $\left(\bar{\varepsilon}_{33}^{s}\right)$ & $13.3 \mathrm{nF} / \mathrm{m}^{-2}$
\end{tabular}


The effect of the load resistance on the tip deflection and output voltage has been further studied in Figure 2.8. As shown in Figure 2.8a, by increasing the load resistance in both short-circuit and open-circuit frequencies, the output voltage increases and converges to a constant voltage. On the other hand, the variation of the tip deflection of the beam versus load resistance in short-circuit and open-circuit frequencies has been shown in Figure 2.8b. As shown in Figure 2.8b, by increasing load resistance in short-circuit and opencircuit frequencies the tip deflection of the beam converges to a constant value. Considering the short-circuit frequency, the output voltage converges to $3.8 \mathrm{mV}$ at $14 \mathrm{k} \Omega$. However, in open-circuit frequency it converges to $470 \mathrm{mV}$ at $100 \mathrm{M} \Omega$. In contrast, as Figure $2.8 \mathrm{~b}$ shows, by increasing load resistance the vibration response at the tip of the beam does not change monotonically for short-circuit or open-circuit frequencies. For excitation at short-circuit frequency, by increasing load resistance the tip deflection decreases, whereas it amplified at open-circuit frequency.

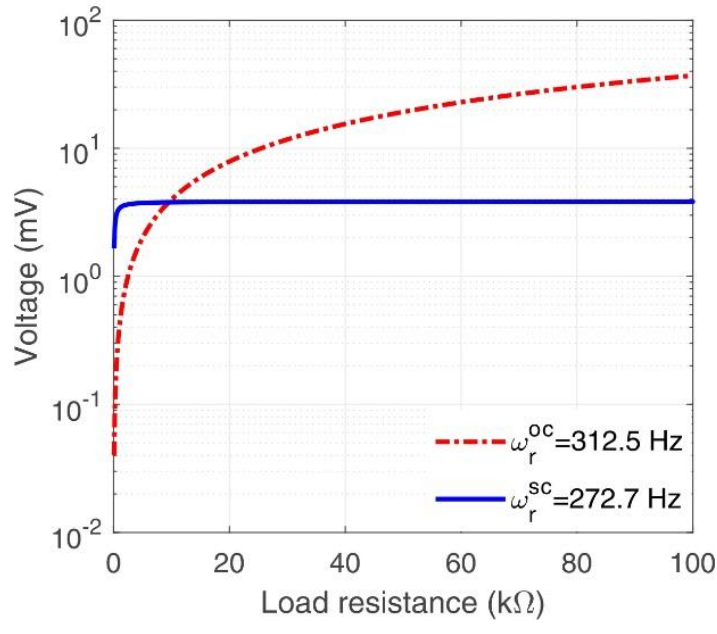

(a)

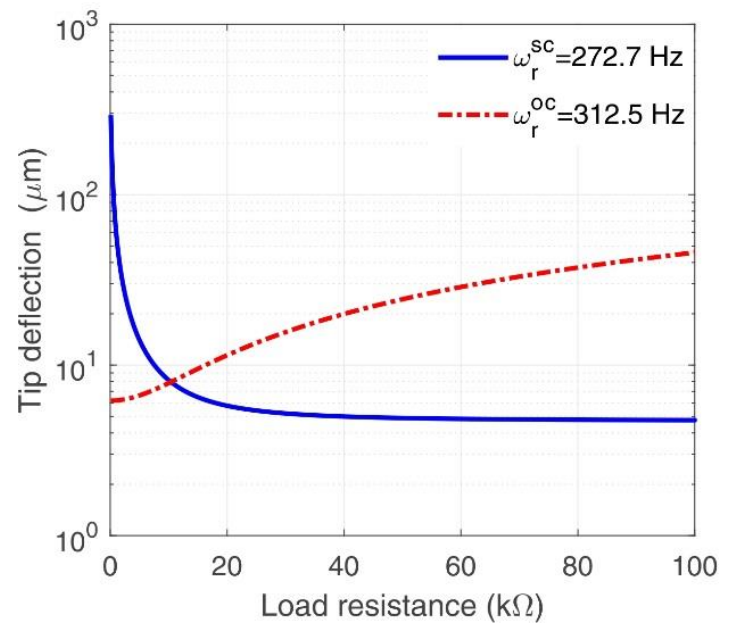

(b)

Figure 2.8 Variation of the tip deflection and output voltage with load resistance for excitations at the short-circuit and open-circuit resonance frequencies.

According to equations (2.33) and (2.34), the output power is proportional to the square of the output voltage, and inversely proportional to the load resistance. Therefore, the output power does not necessarily exhibit monotonic behaviour with increasing (or decreasing) load resistance. 


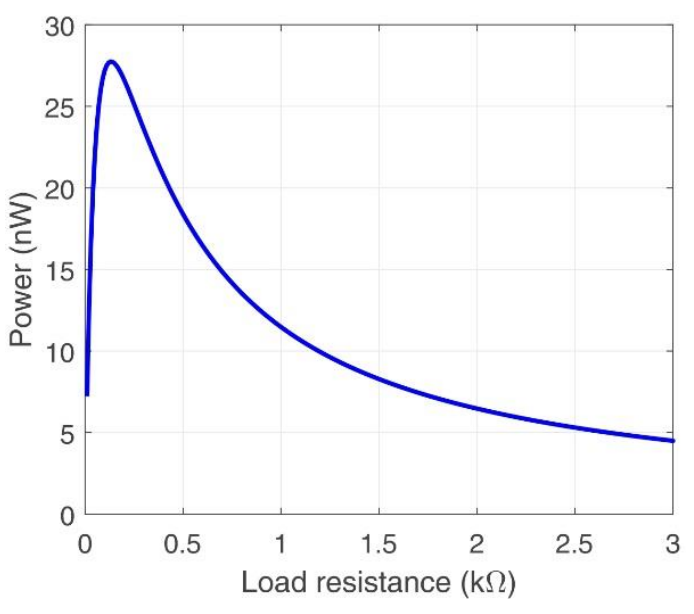

(a)

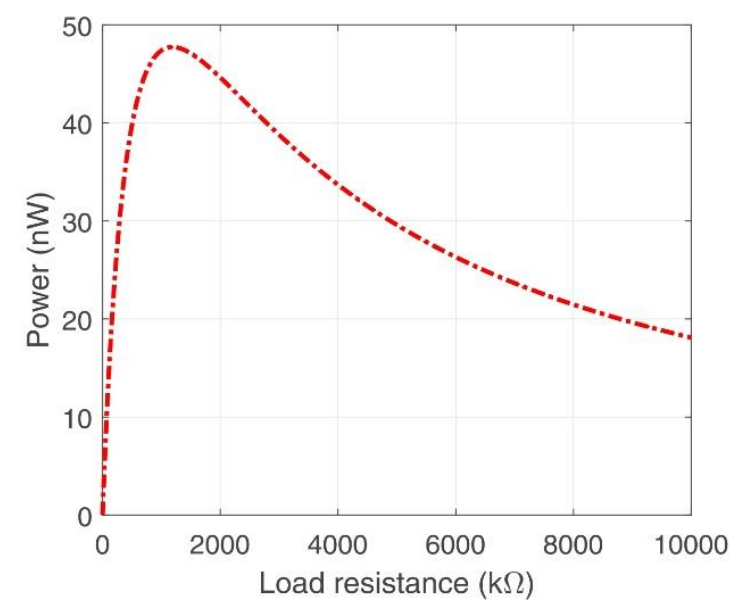

(b)

Figure 2.9 Variation of peak power with load resistance for excitations at (a) the short-circuit and (b) open-circuit resonance frequencies.

The behaviour of output power with changing load resistance for short-circuit and opencircuit frequencies has been given in Figure 2.9. As Figure 2.9a shows, the maximum power in short circuit condition can be obtained at $0.13 \mathrm{k} \Omega$, whereas as shown in Figure $2.9 \mathrm{~b}$ at open-circuit condition the maximum power is delivered when a resistive load of 1.2 $\mathrm{M} \Omega$ is connected to the harvester. The maximum output power of the series and parallel connection cases are the same, but they correspond to different values of optimal load resistance. Furthermore, the optimal load resistance not necessarily the same for all modes of vibration and it can be changed depending on the vibration mode [90]. 


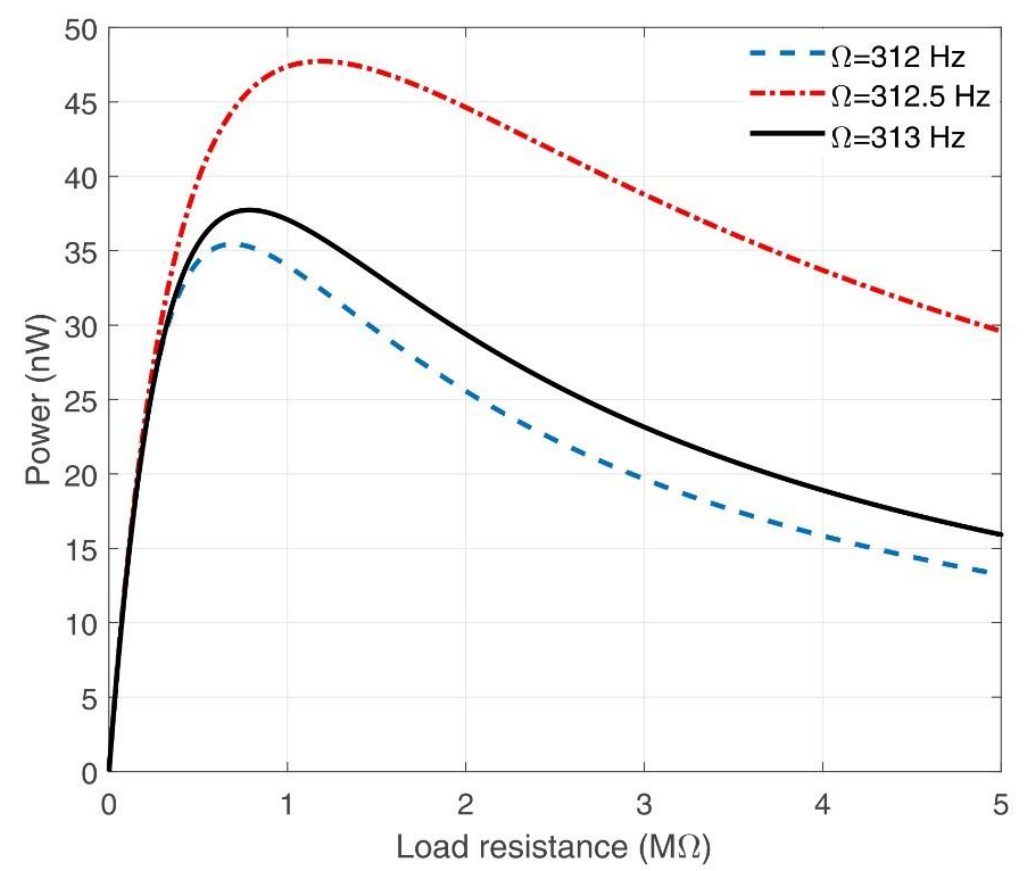

Figure 2.10 Variation of peak power with load resistance for different excitation frequencies

The output power of linear energy harvesters is quite sensitive to the excitation frequency. In resonance frequency, they can harvest maximum power using the optimal load resistance. However, as shown in Figure 2.10, any mismatch between the resonance frequency of the harvester and the excitation frequency can adversely affect the total harvested power. 


\subsection{Closure}

In this chapter, the dynamic behaviour of a MEMS piezoelectric harvester has been studied. The steady-state response of the beam and the steady-state voltage across the load resistance were obtained and the results validated by a reference book written by Erturk and Inman [90]. It was illustrated that depending on the load resistance connected to the piezoelectric layers, the resonance frequency of the harvetser can be changed between short-circuit and open-circuit frequencies. In addition, it was shown that by increasing load resistance the output volatge increases monotonically wheras the amplitude of vibration is decreased in the short-circuit condition (original resonance frequency) and amplified considerably at the open-circuit resonance frequency. Furthermore, the results showed that by increasing load resistance, both output voltage and amplitude of vibration are converged to constant values at short-circuit and opencircuit conditions. The effect of different parameters in the resonance frequency shift were discussed and based on the obtained results, it was observed that the resonance frequency shift $\left(\Delta \omega_{r}=\omega_{r}^{o c}-\omega_{r}^{s c}\right)$ is mostly related to the effect of the piezoelectric layers and electrical parameters. It was also shown that the output power of the harvester can be maximised by using the optimal resisctance. However, any mismatch between the resonance frequency of the harvester and the excitation frequency can decrease the harvested power significantly. To overcome this problem, in the next chapter we propose a MEMS piezoelectric harvester with adjustable resonance frequency. 


\section{Chapter 3: Modelling and design of MEMS piezoelectric energy harvester with adjustable resonance frequency}

This chapter presents a comprehensive study for the modelling and design of a MEMS piezoelectric harvester which employs an electrostatic device to adjust its resonance frequency. The idea is demonstrated in a hybrid system consisting of a cantilevered piezoelectric harvester combined with an electrostatic harvester which is connected to a variable voltage source. The main motivation of the proposed model is to control the resonance frequency of the MEMS piezoelectric harvester with the DC voltage applied to the electrostatic system in order to maximize the harvested power.

\subsection{Model description and mathematical modelling}

Figure 3.1 shows the model proposed in this chapter. The model is an isotropic microbeam of length $L$, width $b$, thickness $h$, density $\rho_{\tilde{s}}$ and Young's modulus $E_{\tilde{S}}$, sandwiched between piezoceramic layers of thickness $h_{\tilde{p}}$, Young's modulus $c_{11}^{E}$ and density $\rho_{\tilde{p}}$ throughout the microbeam length and located between two electrodes (electrode 1 and electrode 2). As illustrated in Figure 3.1, the piezoceramic layers are connected to the resistance $(R)$ in parallel connection and the coordinate system is attached to the middle of the left end of the microbeam where $x$ and $z$ refer to the horizontal and vertical coordinates respectively. The tip mass is used to control the dynamics of the micro-cantilever. 


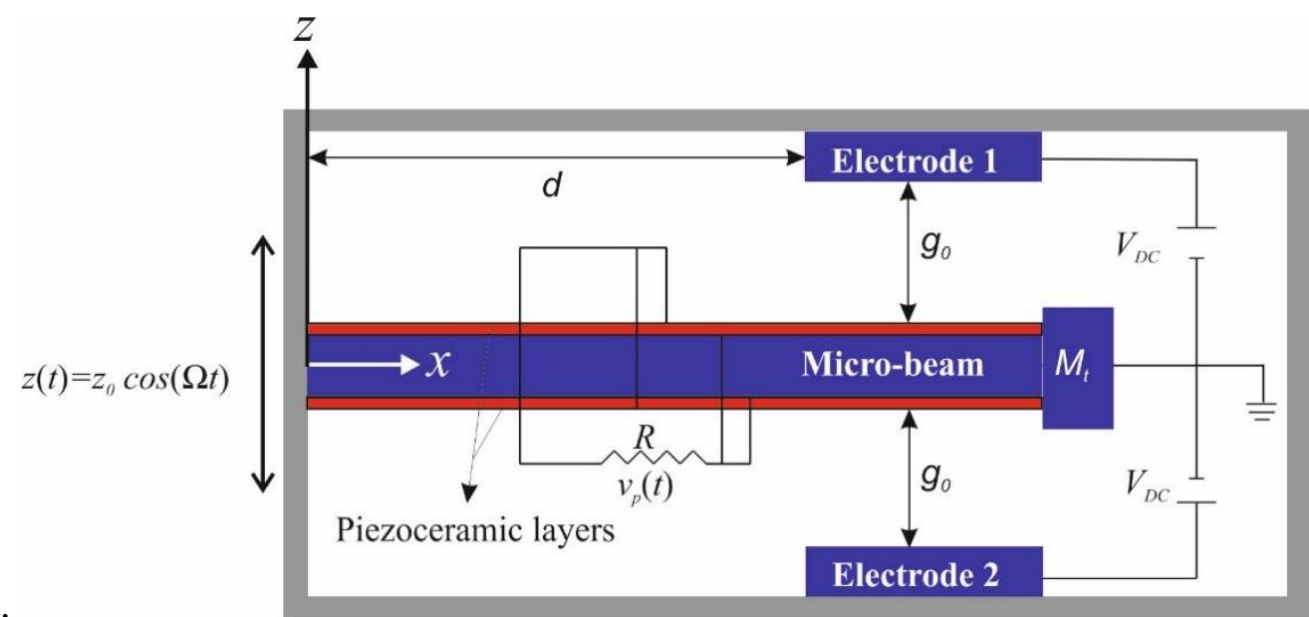

Figure 3.1 Schematic of the proposed energy harvester.

The governing equation of transverse motion can be written as

$$
\begin{gathered}
E I \frac{\partial^{4} w_{r e l}^{p}}{\partial x^{4}}+m \frac{\partial^{2} w_{r e l}^{p}}{\partial t^{2}}+c_{a} \frac{\partial w_{r e l}^{p}}{\partial t}-\vartheta_{p} v_{p}(t)\left(\frac{d \delta(x)}{d x}-\frac{d \delta(x-L)}{d x}\right) \\
=F_{e s}\left(V_{D C}, w_{r e l}^{p}\right)-\left(m+M_{t} \delta(x-L)\right) \frac{\partial^{2} z(t)}{\partial t^{2}}
\end{gathered}
$$

where $E I, m$ and $\vartheta_{p}$ are given by equations (2.10) and (2.11). Equation (3.1) is subject to the following boundary conditions

$$
\begin{gathered}
w_{r e l}^{p}(0, t)=0, \quad \frac{\partial w_{r e l}^{p}(0, t)}{\partial x}=0, \\
\frac{\partial}{\partial x}\left(E I \frac{\partial^{2} w_{r e l}^{p}(L, t)}{\partial x^{2}}\right)=M_{t}\left(\frac{\partial^{2} w_{r e l}^{p}(L, t)}{\partial t^{2}}\right) \\
E I \frac{\partial^{2} w_{r e l}^{p}(L, t)}{\partial x^{2}}=-I_{M_{t}} \frac{\partial^{2}}{\partial t^{2}}\left(\frac{\partial w_{r e l}^{p}(L, t)}{\partial x}\right)
\end{gathered}
$$

In equation (3.1), $w_{\text {rel }}^{p}$ is the transverse deflection of the beam relative to its base at the position $x$ and time $t, c_{a}$ is the viscous air damping coefficient, $\delta(x)$ is the Dirac delta function, $F_{e s}$ is the electrostatic force between electrodes which is a function of applied DC voltage to the microbeam, and $w_{r e l}^{p}, z(t)$ is the base excitation function, $v_{p}(t)$ is the voltage across the electrodes of each piezoceramic layer and $\vartheta_{p}$ is the coupling. The electrical circuit equation based on Kirchhoff's laws can be expressed as 


$$
C_{\tilde{p}} \frac{d v_{p}(t)}{d t}+\frac{v_{p}(t)}{2 R}+i_{\tilde{p}}^{p}(t)=0
$$

where the internal capacitance $\left(C_{\tilde{p}}\right)$ and the current source are given by equation $(2.21)$. Using the electrostatic principle, the force between electrodes and the microbeam can be written as follows

$$
F_{e s}=\frac{\varepsilon_{0} b H(x-d)}{2}\left(\frac{V_{D C}^{2}}{\left(g_{0}-w_{\text {rel }}^{p}\right)^{2}}-\frac{V_{D C}^{2}}{\left(g_{0}+w_{\text {rel }}^{p}\right)^{2}}\right)
$$

In equation (3.4), $\varepsilon_{0}$ is the permittivity of free space, $H(x)$ is the Heaveside function, $V_{D C}$ is the applied DC voltage to the microbeam, $g_{0}$ is the air gap between electrodes (the system is assumed to be symmetrical). It is obvious that the electrostatic force is an inherent source of nonlinearity. In order to investigate the nonlinearity, the total deflection of the microbeam can be considered as

$$
w_{r e l}^{p}(x, t)=w_{s t}(x)+w_{\mathrm{d}}(x, t)
$$

which is the summation of static $\left(w_{s t}\right)$ and dynamic $\left(w_{d}\right)$ deflection of the beam. By Substituting equation (3.5) into equation (3.4) and using a Taylor series expansion about the equilibrium position $\left(w_{s t}=0\right)$ up to the ninth-order, the electrostatic force $\left(F_{e s}\right)$ can be approximated as follows

$$
F_{e s}=\frac{\varepsilon_{0} b V_{D C}^{2} H(x-d)}{2}\left(\frac{4 w_{d}}{g_{0}^{3}}+\frac{8 w_{d}^{3}}{g_{0}^{5}}+\frac{12 w_{d}^{5}}{g_{0}^{7}}+\frac{16 w_{d}^{7}}{g_{0}^{9}}+\frac{20 w_{d}^{9}}{g_{0}^{11}}+\cdots\right)
$$

It is noticeable that the number of terms in the electrostatic force approximation depends on the different parameters such as amplitude of base excitation and damping coefficient. Considering equation (3.6) and using the Galerkin decomposition method, the spatial dependence can be eliminated in equation (3.1). To this end, the deflection of the microbeam can be represented as a series expansion in terms of the eigenfunctions of the microbeam, i.e.

$$
\mathrm{w}_{\mathrm{d}}(x, t)=\sum_{i=1}^{N} U_{i}(t) \varphi_{i}(x)
$$


where $\varphi_{i}(x)$ is the $i$ th linear undamped mode shape of the straight microbeam and $U_{i}(t)$ is the $i$ th generalized coordinate. By considering the approximated electrostatic force and using the Galerkin method, equations (3.1) and (3.4) can be converted into a system of differential equations. Considering a single-mode approximation $(\mathrm{N}=1)$ yields the following equations

$$
\begin{gathered}
M \ddot{U}(t)+C \dot{U}(t)+\left(K_{m}+K_{e}\right) U(t)-\theta_{p} v_{p}(t)+K_{n 1} U^{3}(t)+K_{n 2} U^{5}(t) \\
+K_{n 3} U^{7}(t)+K_{n 4} U^{9}(t)=F_{b} \cos (\Omega t) \\
\dot{v}_{p}(t)+\lambda v_{p}(t)+\kappa \dot{U}(t)=0
\end{gathered}
$$

where

$$
\begin{aligned}
& K_{e}=a_{1} \int_{0}^{L} \varphi^{2}(x) H(x-d) d x, \quad \theta_{p}=\vartheta_{p} \frac{d \varphi(L)}{d x} \\
& K_{n 1}=a_{2} \int_{0}^{L} \varphi^{4}(x) H(x-d) d x, \quad \kappa=\frac{\bar{e}_{31} b\left(h_{\tilde{p}}+h_{\tilde{s}}\right)}{2 C_{p}}\left(\frac{d \varphi(L)}{d x}\right) \\
& K_{n 2}=a_{3} \int_{0}^{L} \varphi^{6}(x) H(x-d) d x, \quad \lambda=\frac{1}{2 R C_{p}} \\
& K_{n 3}=a_{4} \int_{0}^{L} \varphi^{8}(x) H(x-d) d x, \quad K_{n 4}=a_{5} \int_{0}^{L} \varphi^{10}(x) H(x-d) d x, \\
& F_{b}=z_{0} \Omega^{2}\left(m \int_{0}^{L} \varphi(x) d x+M_{t} \int_{0}^{L} \varphi(x) \delta(x-L) d x\right)
\end{aligned}
$$

where $a_{1}, a_{2}, a_{3}, a_{4}$ and $a_{5}$ have been given in Appendix A. For convenience, equation (3.8a) can be rewritten as

$$
\begin{gathered}
\ddot{U}(t)+2 \mu \omega_{n} \dot{U}(t)+\omega_{n}^{2} U(t)-\chi v_{p}(t)+\alpha U^{3}(t)+\beta U^{5}(t)+\gamma U^{7}(t) \\
+\delta U^{9}(t)=F \cos (\Omega t)
\end{gathered}
$$


where

$$
\begin{gathered}
\mu=\frac{C}{2 M \omega_{n}}, \quad \omega_{n}=\sqrt{\frac{K_{m}+K_{e}}{M}}, \quad \chi=\frac{\theta_{p}}{M}, \quad \alpha=\frac{K_{n 1}}{M}, \quad \beta=\frac{K_{n 2}}{M}, \\
\gamma=\frac{K_{n 3}}{M}, \quad \delta=\frac{K_{n 4}}{M}, \quad F=\frac{F_{b}}{M}
\end{gathered}
$$

equation (3.10) shows that the electrostatic forces create a negative stiffness which opposes the mechanical stiffness. Above a certain applied DC voltage, electrostatic MEMS devices can become unstable. This voltage is known as the pull-in voltage [93]. To determine the analytical solution of transverse vibration of the mircobeam, the method of harmonic balance is used. By assuming a steady state periodic response, $U(t)$ and $v_{p}(t)$ can be written as

$$
\begin{aligned}
& U(t)=a_{1}(t) \sin (\Omega t)+b_{1}(t) \cos (\Omega t) \\
& v_{p}(t)=a_{2}(t) \sin (\Omega t)+b_{2}(t) \cos (\Omega t)
\end{aligned}
$$

with slowly varying coefficients such as

$$
\begin{gathered}
\dot{U}(t)=\left(\dot{b}_{1}+a_{1} \Omega\right) \cos (\Omega t)+\left(\dot{a}_{1}-b_{1} \Omega\right) \sin (\Omega t) \\
\dot{v}_{p}(t)=\left(\dot{b}_{2}+a_{2} \Omega\right) \cos (\Omega t)+\left(\dot{a}_{2}-b_{2} \Omega\right) \sin (\Omega t) \\
\ddot{U}(t)=\Omega\left(2 \dot{a}_{1}-b_{1} \Omega\right) \cos (\Omega t)-\Omega\left(2 \dot{b}_{1}+a_{1} \Omega\right) \sin (\Omega t)
\end{gathered}
$$

Substituting the above expressions into equations (3.9) and (3.10), neglecting higher harmonics and balancing terms multiplied by $\sin (\Omega t)$ and $\cos (\Omega t)$ from the mechanical equation, the following equations are obtained

$$
\begin{gathered}
2 \mu \omega_{n} \dot{a}_{1}-2 \Omega \dot{b}_{1}=Q a_{1}+\chi a_{2}+2 \mu \omega_{n} b_{1} \Omega \\
2 \mu \omega_{n} \dot{b}_{1}+2 \Omega \dot{a}_{1}=Q b_{1}+\chi b_{2}+F-2 \mu \omega_{n} a_{1} \Omega
\end{gathered}
$$

where

$$
r^{2}=a_{1}^{2}+b_{1}^{2}
$$




$$
Q=-\omega_{n}^{2}+\Omega^{2}-\frac{3}{4} \alpha r^{2}-\frac{5}{8} \beta r^{4}-\frac{35}{64} \gamma r^{6}-\frac{63}{128} \delta r^{8}
$$

Applying the same approach to the electrical equation yields

$$
\begin{gathered}
\kappa \dot{a}_{1}+\dot{a}_{2}=b_{2} \Omega-\lambda a_{2}+\kappa b_{1} \Omega \\
\kappa \dot{b}_{1}+\dot{b}_{2}=-a_{2} \Omega-\lambda b_{2}-\kappa a_{1} \Omega
\end{gathered}
$$

In steady state, all time derivatives vanish so that we can re-write the mechanical amplitude equations as

$$
\begin{gathered}
0=Q a_{1}+\chi a_{2}+2 \mu \omega_{n} b_{1} \Omega \\
0=Q b_{1}+\chi b_{2}+F-2 \mu \omega_{n} a_{1} \Omega
\end{gathered}
$$

and the electrical amplitude equations as

$$
\begin{aligned}
& 0=b_{2} \Omega-\lambda a_{2}+\kappa b_{1} \Omega \\
& 0=a_{2} \Omega+\lambda b_{2}+\kappa a_{1} \Omega
\end{aligned}
$$

The electrical coefficients $a_{2}$ and $b_{2}$ can be obtained from equations (3.25) and (3.26) as

$$
\begin{aligned}
& a_{2}=\frac{\kappa \Omega}{\Omega^{2}+\lambda^{2}}\left(\lambda b_{1}-a_{1} \Omega\right) \\
& b_{2}=-\frac{\kappa \Omega}{\Omega^{2}+\lambda^{2}}\left(\lambda a_{1}+b_{1} \Omega\right)
\end{aligned}
$$

Substituting the steady-state solutions for $a_{2}$ and $b_{2}$ into the steady-state equations for $a_{1}$ and $b_{1}$ yields

$$
\begin{aligned}
& 0=\Lambda_{a} a_{1}+\Lambda_{b} b_{1} \\
& F=\Lambda_{b} a_{1}-\Lambda_{a} b_{1}
\end{aligned}
$$


where

$$
\Lambda_{a}=Q-\frac{\kappa \chi \Omega^{2}}{\Omega^{2}+\lambda^{2}}, \quad \Lambda_{b}=2 \mu \omega_{n} \Omega+\frac{\kappa \chi \lambda \Omega}{\Omega^{2}+\lambda^{2}}
$$

Squaring and adding equations (3.29) and (3.30) gives an eighteenth order nonlinear algebraic equation in $r$ as

$$
F^{2}=r^{2}\left(\Lambda_{a}^{2}+\Lambda_{b}^{2}\right)
$$

and the frequency response can be determined by numerically finding the positive real roots of equation (3.32). Similarly, by squaring and adding equations (3.27) and (3.28), the response voltage amplitude can be written in terms of the mechanical amplitude as

$$
S=r \sqrt{\Gamma_{a}^{2}+\Gamma_{b}^{2}}
$$

where

$$
\Gamma_{a}=\frac{\kappa \lambda \Omega}{\Omega^{2}+\lambda^{2}}, \quad \Gamma_{b}=\frac{\kappa \Omega^{2}}{\Omega^{2}+\lambda^{2}}
$$

and $r$ is an implicit function of the forcing amplitude, damping, electromechanical coupling, and electrical dissipation as derived from the roots of equation (3.32). The peak power through the resistance can then be written as

$$
P_{0}=\frac{S^{2}}{R}
$$

and the average power is

$$
\bar{P}_{0}=\frac{S^{2}}{2 R}
$$

In this subsection a stability analysis is provided because only a few solutions of the eighteen total roots for $r$ in equation (3.32) can be realized in practice. In order to ascertain the stability of the solutions, it is first necessary to rewrite equations (3.17), (3.18), (3.21) and (3.22) in matrix form

$$
\mathbf{A} \dot{\mathbf{x}}=\mathbf{D}(\mathbf{x})
$$


where

$$
\mathbf{A}=\left[\begin{array}{cccc}
2 \mu \omega_{n} & -2 \Omega & 0 & 0 \\
2 \Omega & 2 \mu \omega_{n} & 0 & 0 \\
\kappa & 0 & 1 & 0 \\
0 & \kappa & 0 & 1
\end{array}\right], \quad \mathbf{D}(\mathbf{x})=\left[\begin{array}{c}
Q a_{1}+\chi a_{2}+2 \mu \omega_{n} b_{1} \Omega \\
Q b_{1}+\chi b_{2}+F-2 \mu \omega_{n} a_{1} \Omega \\
b_{2} \Omega-\lambda a_{2}+\kappa b_{1} \Omega \\
-a_{2} \Omega-\lambda b_{2}-\kappa a_{1} \Omega
\end{array}\right]
$$

with the vector $\mathbf{x}=\left[\begin{array}{lll}a_{1} b_{1} & a_{2} b_{2}\end{array}\right]^{\mathrm{T}}$. One may write equation (3.37) as

$$
\dot{\mathbf{x}}=\mathbf{G}(\mathbf{x})
$$

where $\mathbf{G}(\mathbf{x})=\mathbf{A}^{-1} \mathbf{D}(\mathbf{x})$. The stability can be investigated by constructing the Jacobian matrix of $\mathbf{G}(\mathbf{x})$ and calculating its value at the steady state values for $\mathbf{x}$, which are indicated as $\mathbf{x}_{\mathrm{SS}}$

$$
\mathbf{J}=\left.\frac{\partial \mathbf{G}}{\partial \mathbf{x}}\right|_{\mathbf{x}=\mathbf{x}_{\text {ss }}}
$$

The values for $\mathbf{x}_{\mathrm{ss}}$ can be found through the solutions of equations (3.23), (3.24), (3.25) and (3.26). By evaluating the eigenvalues of the Jacobian matrix, the stability of the steady state solutions is determined. If all of the eigenvalues associated with the steady state solution have negative real parts, then the solution is asymptotically stable. Otherwise, if one eigenvalue has a positive real part, then the solution is unstable.

\subsection{Numerical Results and Discussion}

In order to study the behaviour of the proposed model, a clamped-free microbeam is considered with the same characteristics given in Table 2.1. The results will be discussed in the following subsections. At first, a parametric study is presented to analyse the effect of different parameters in the performance of the harvester. Afterwards, the sustainability of the proposed model will be investigated and finally a tuneable MEMS piezoelectric harvester is designed to cover a given range of vibration source.

\subsubsection{Parametric study}

This subsection investigates the effect of different parameters in the design of a tuneable harvester. Based on the proposed model, electrostatic forces create a negative stiffness, 
which decreases the equivalent stiffness of the structure. Generally, electrostatic stiffness increases by increasing the applied DC voltage or/and decreasing initial gaps between electrodes. Based on the Taylor expansion of this force at a constant initial gap, by increasing the applied DC voltages to the electrostatic electrodes, the electrical stiffness of the structure is increased and leads to the decrease of the equivalent stiffness of the structure (see equation (3.8a)). Consequently, at the critical value of the applied DC voltage called the pull-in voltage, the system has a static instability by undergoing a pitchfork bifurcation [47]. Figure 3.2 shows that increasing the air gap between the electrodes decreases the electrostatic force and increases the pull-in voltage.

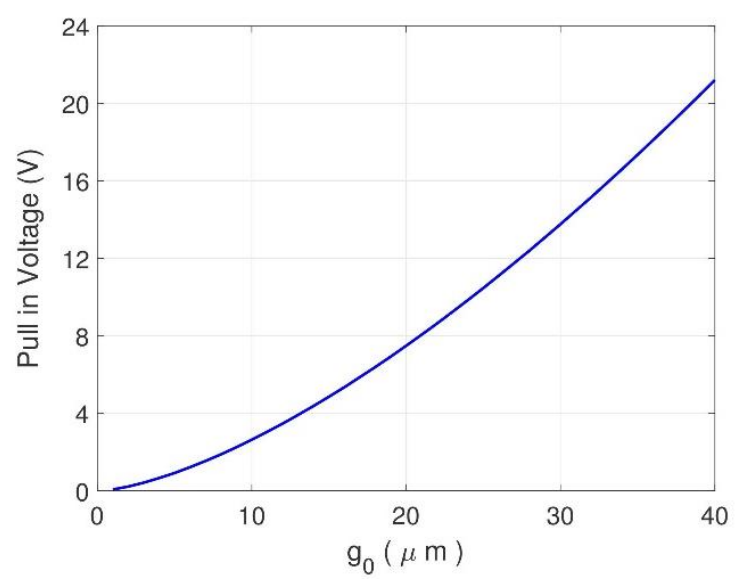

(a)

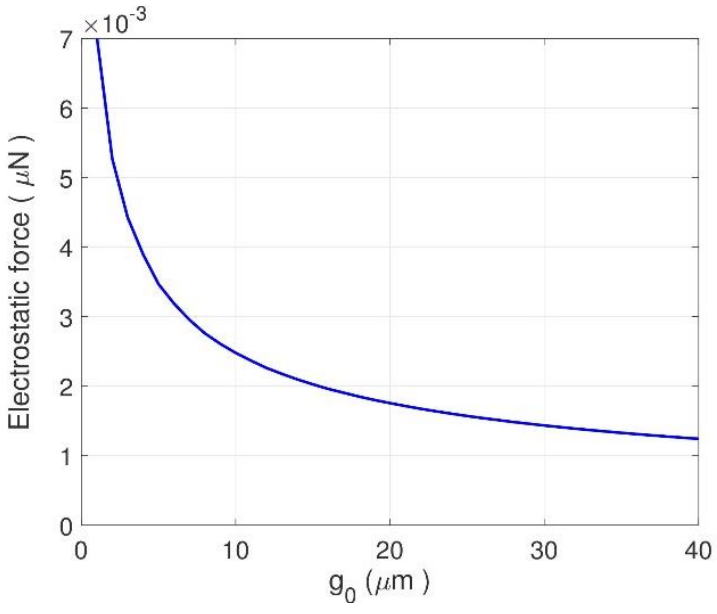

(b)

Figure 3.2 Variation of the (a) pull in voltage and (b) the electrostatic force with the air gap between the electrodes $\left(g_{0}\right)$.

On the other hand, decreasing the equivalent stiffness of the structure decreases the resonance frequency of the system. Figure 3.3 illustrates that by considering different air gaps between electrodes the short-circuit resonance frequency of the system decreases with increasing DC voltage and becomes zero at the pull-in voltage. 


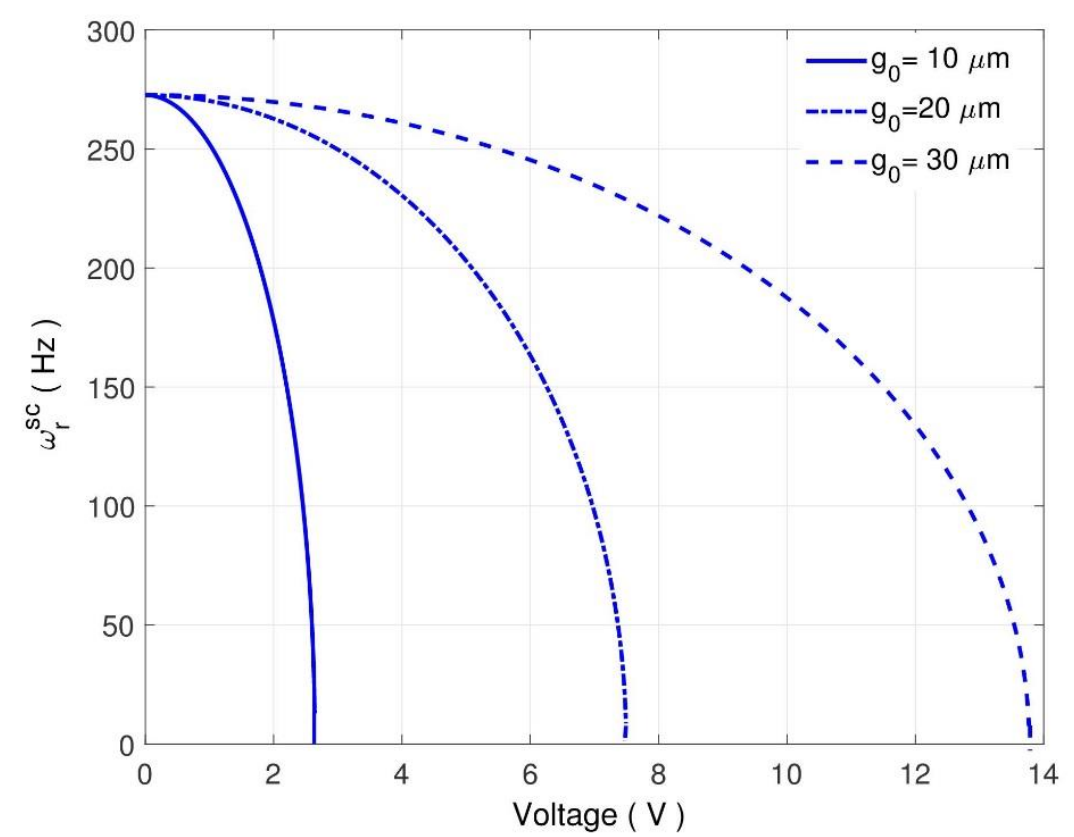

Figure 3.3 Variation of the natural frequency with electrostatic voltage for different air gaps when $L_{e}=0.5 \mathrm{~L}$.

The dynamic behaviour of the system is also affected by the nonlinearity of the electrostatic force. In order to study the dynamic analysis of the harvester using the harmonic balance method (HBM), the electrostatic force is approximated by a Taylor series expansion. In addition, an arbitrary case $\left(g_{0}=30 \mu \mathrm{m}, L_{e}=0.5 L, V_{D C}=8 \mathrm{~V}\right.$, $z_{0}=0.3 \mu \mathrm{m}$ and $R=100 \mathrm{k} \Omega$ ) has been considered to show the results. It is noticeable that some of the parameters of the considered case may change in some figures. Figure 3.4 illustrates that acceptable convergence can be obtained by including the terms up to ninth-order. 


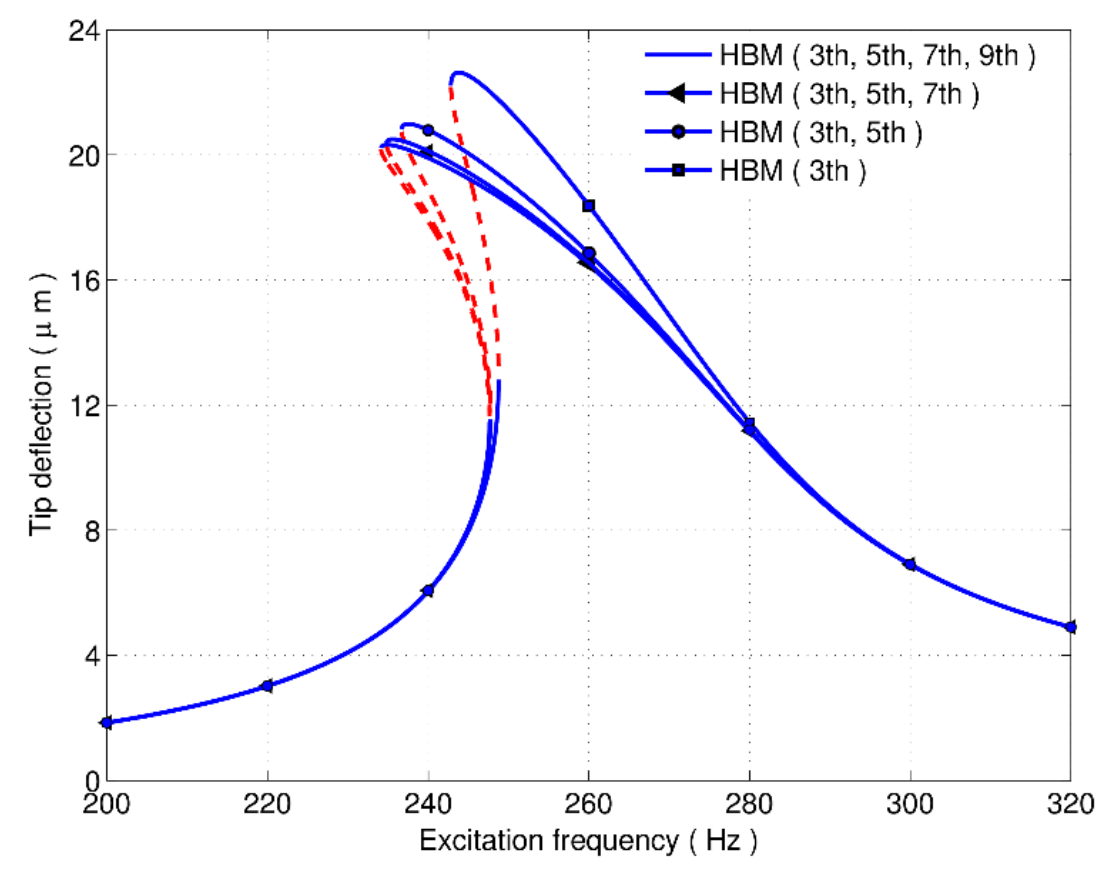

Figure 3.4 Displacement frequency response curve for different order of nonlinearity

The presented results based on the ninth order nonlinearity are verified by numerical results. Figure 3.5 shows a good agreement between the results obtained by harmonic balance and those achieved by time integration (Runge-kutta forth-order method). In the numerical method, the exact form of the electrostatic force is considered. According to the dynamic analysis of the system the peak output power can be obtained from equation (3.35). Adjusting the resonance frequency of the system to match the frequency of the base excitation will increase the harvested power. Also, choosing the appropriate resistance can increase the harvested power. As illustrated in Figure 3.6a, the optimal resistance depends on the frequency of base excitation and Figure 3.6b shows that the optimal resistance reduces as the frequency of the base excitation increases. 


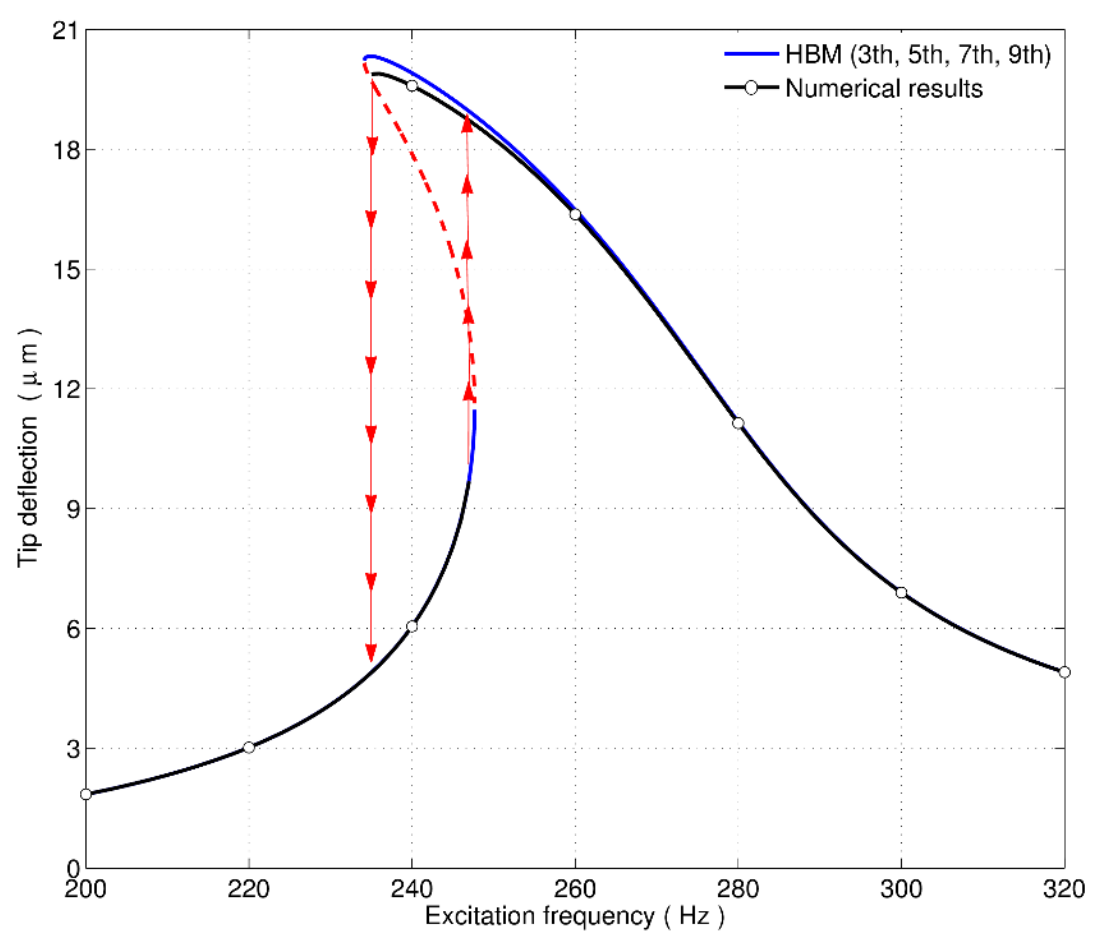

Figure 3.5 Displacement frequency response curve

According to the dynamic analysis of the system, the peak output power can be obtained from equation (3.35). Adjusting the resonance frequency of the system to match the frequency of the base excitation will increase the harvested power. Also, choosing the appropriate resistance can increase the harvested power. As illustrated in Figure 3.6a, the optimal resistance depends on the frequency of base excitation and Figure 3.6b shows that the optimal resistance reduces as the frequency of the base excitation increases.

The dynamic behaviour of the system can be affected by the applied DC voltage, the length of the electrode, the initial gap and the resistance. Therefore, these parameters can significantly affect the dynamic behaviour of the system. Increasing the length of the electrodes or/and decreasing initial gap at constant voltage increases softening effect. However, it will decrease the harvested power. 


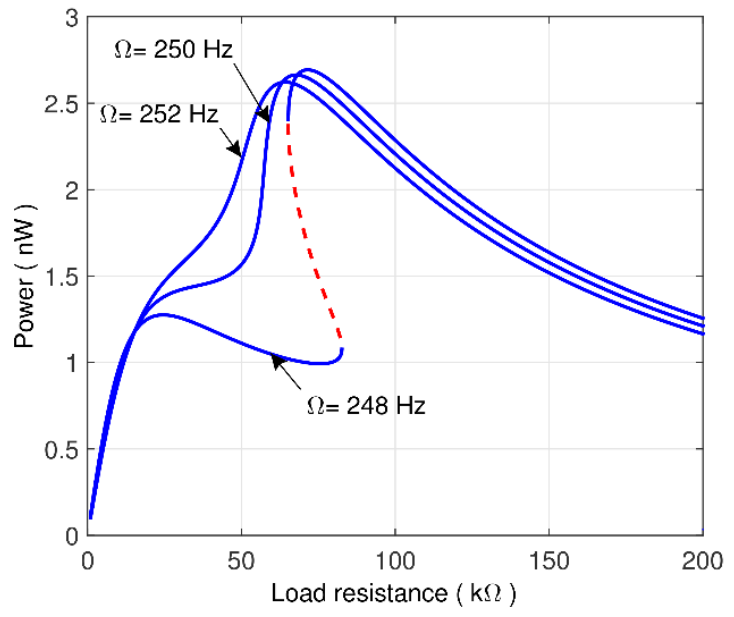

(a)

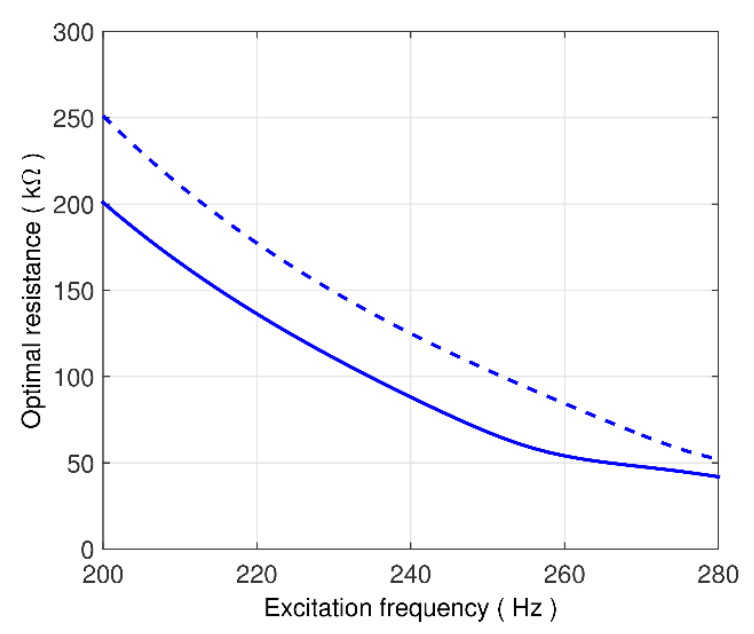

(b)

Figure 3.6 (a)Variation of the piezoelectric peak power with load resistance for different frequencies of base excitation (b) Variation of the optimal resistance for different frequencies of base excitation

Figure 3.7 investigates the effect of electrode length and resistance on the dynamics of the system at constant applied DC voltage. As shown in Figure 3.7a, the length of the electrode is a key parameter in changing the resonance frequency of the system and also the nonlinearity of the system is affected by this parameter. Moreover, changing the value of the resistance changes the nonlinearity of the system (see Figure 3.7b). Therefore, by considering constant values for initial gap and electrodes length, the resonance frequency of the harvester can be controlled by the applied DC voltage and resistance to maximize the harvested power.

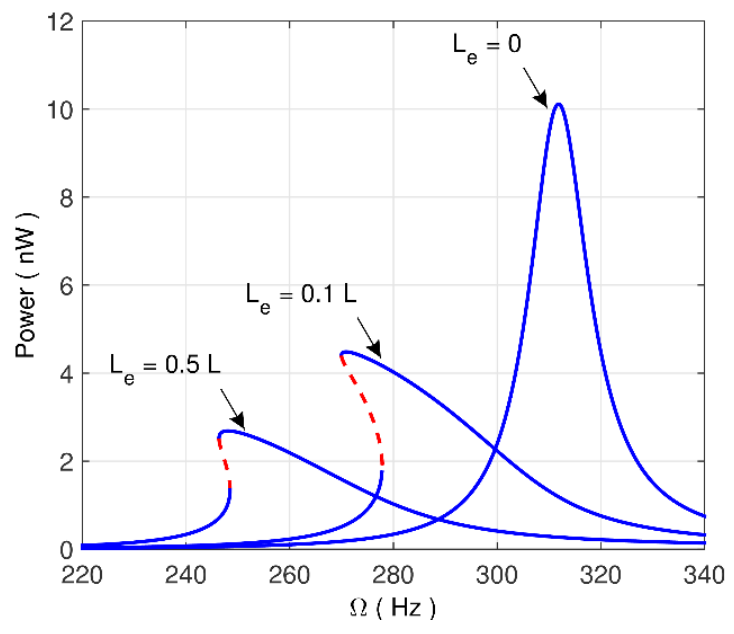

(a) $R=70 \mathrm{k} \Omega$

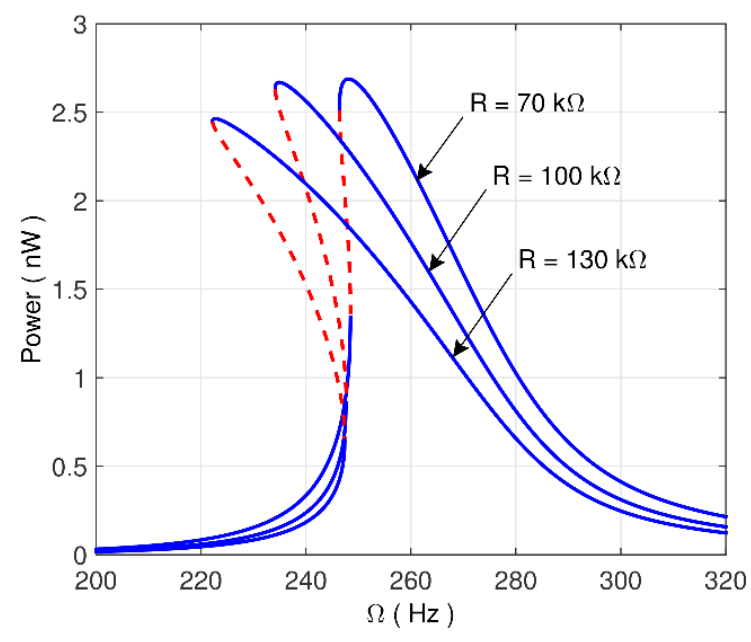

(b) $L_{e}=0.5 \mathrm{~L}$

Figure 3.7 Variation of the piezoelectric peak power with frequency of base excitation 
Increasing the range of operation frequency of the harvester based on the increasing applied DC voltage and the length of the electrode is limited due to the pull-in instability. However increasing the electrostatic force decreases the vibration of the microbeam and leads to a decrease in the output power. Therefore, the system needs to be optimized for maximum harvested power. On the other hand, due to the softening nonlinearity there are three solutions for the beam response within the frequency range closed to resonance and in order to harvest maximum power the beam response should always occur at the higher of the two solutions and close to resonance (but not too close to risk jumping down to the low amplitude solution). However, the response at the high amplitude solution mostly depends on the initial conditions and hence it cannot be guaranteed. The control system using the applied DC voltage can be used to ensure the harvester always responds in the higher amplitude solution. For a given excitation frequency, if the harvester response happens to be in the lower amplitude solution, the DC voltage is increased until a region is reached where the harvester only has a single solution. The DC voltage is then slowly reduced, and the harvester follows the high amplitude solution until the resonance is obtained.

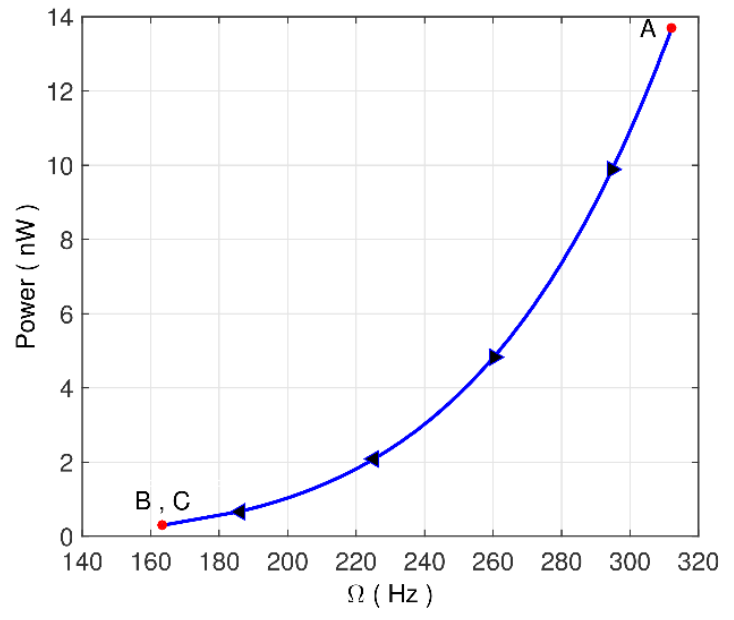

(a)

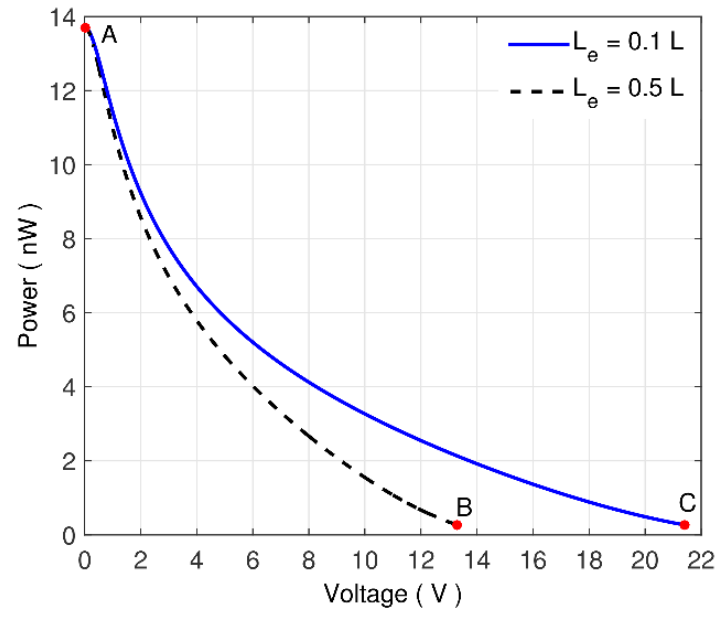

(b)

Figure 3.8 (a) Frequency range of the harvester based on increasing DC voltage (b)

Voltage range for different lengths of electrodes to cover the frequency range

Figure 3.8a shows that electrostatic forces, a frequency range between $312.2 \mathrm{~Hz}$ to 163.4 $\mathrm{Hz}$ is accessible. However, the amount of variation in the voltages that requires covering the given range of operation frequency will be dependent upon the length of the electrodes, as shown in Figure 3.8b. 
In some cases, it is possible to have a vibration source with a constant frequency and a variable amplitude of base excitation. Figure 3.9 investigates the variation in the output power when the excitation amplitude is changed at a constant frequency. This figure shows that by increasing the amplitude of the base excitation, the harvested power increases. However, depending on the frequency of the vibration source, jumping is observed in some excitation amplitudes ranges.

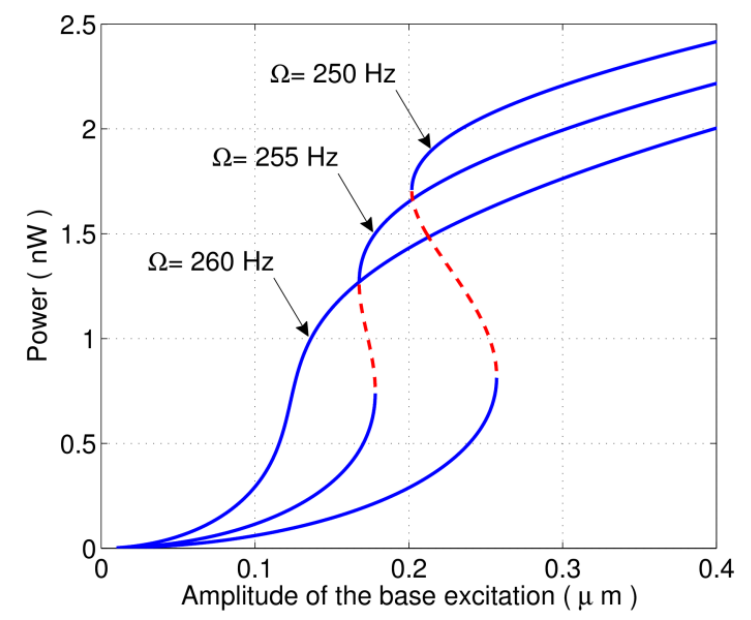

Figure 3.9 Variation of the piezoelectric peak power with the frequency of base excitation.

\subsubsection{Sustainability of the proposed harvester}

In the proposed model the voltage source has been used to change the natural frequency of the harvester. However, this source can be charged by the harvested power from the electrostatic harvester. In general, electrostatic harvesters have passive structures which need an energy cycle to convert mechanical energy into electrical energy [94]. As discussed in Chapter 1, there are two common cycles which use charge or voltage constraint concepts in the conversion cycle. Figure 3.1 shows that the proposed model consists of two variable capacitors which are connected to the same DC voltage [56]. The variable capacitors are charged by $V_{D C}$ and their capacitances can be expressed as

$$
C_{v 1}(t)=\frac{\varepsilon_{0} A_{e}}{\left(g_{0}+w_{a v}(t)\right)}, \quad C_{v 2}(t)=\frac{\varepsilon_{0} A_{e}}{\left(g_{0}-w_{a v}(t)\right)}
$$


where $A_{e}$ is the overlapping area between the fixed and moveable electrodes and $w_{a v}$ is the average value of $w$ in this area. The total amount of energy stored in the capacitors is

$$
U_{t}(\mathrm{t})=\frac{1}{2}\left(C_{v 1}(\mathrm{t})+C_{v 2}(\mathrm{t})\right) V_{D C}^{2}
$$

and consequently, the generated instantaneous power can be represented by

$$
P_{t}(t)=\frac{d U_{t}}{d t}=\frac{\varepsilon_{0} A_{e} V_{D C}^{2}}{2}\left(\frac{1}{\left(g_{0}-w_{\mathrm{av}}(t)\right)^{2}}-\frac{1}{\left(g_{0}+w_{\mathrm{av}}(t)\right)^{2}}\right) \frac{d w_{\mathrm{av}}}{d t}
$$

As illustrated in Figure 3.10, the instantaneous power obtained from the capacitors varies between positive and negative values during each cycle. Therefore, the voltage source is self-chargeable.

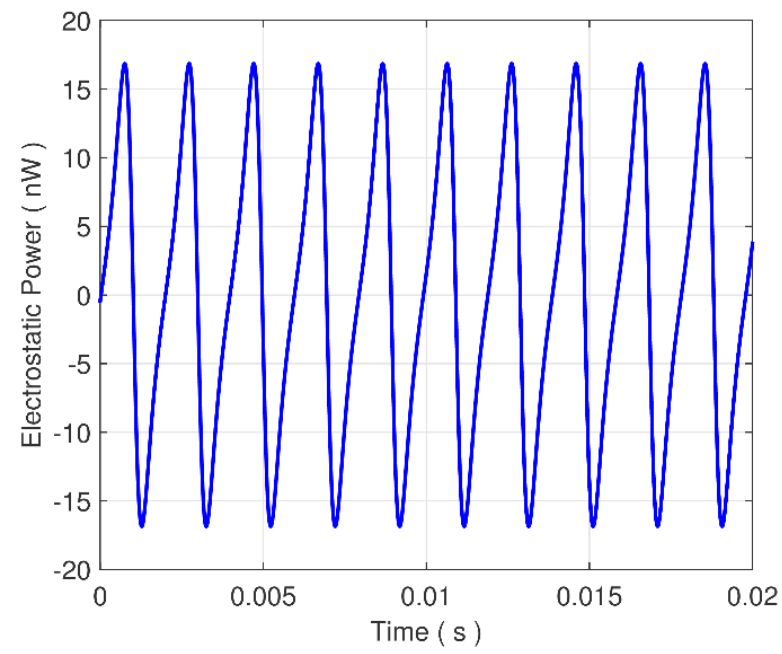

Figure 3.10 Variation of the instantaneous electrostatic power with time when $L_{e}=$

$$
0.01 \mathrm{~L} \text { and } \Omega=253 \mathrm{~Hz}
$$

In real life applications, they are some power losses in the electrical circuit which generally come in the form of conduction and switching losses. The calculation of conduction losses is relatively simple but the calculation of switching is dependent on a number of factors such as parasitic effect, impedance of the gate drive circuit and temperature. On the other hand, deformation of the mechanical structure of the harvester results in charges with opposite polarities appearing on same surface, leading to current loss in the attached metal electrode. Considering all these effects we can calculate the energy conversion efficiency. 


\subsubsection{Design strategy}

The design of the tuneable harvester should be preceded by an analysis of the vibration source. There are two main design considerations: the first is related to the maximum value of the excitation frequency $\left(\Omega_{\max }\right)$ which is important when choosing the dimensions of the microbeam to match the resonance frequency of the harvester with $\Omega_{\max }$. The pull-in instability and the clearance between the electrodes are the second design factor. In addition, because of the strong coupling between the mechanical and electrical equations, resistance can significantly change the mechanical behaviour of the harvester. Therefore, this resistance is a key parameter in analysing the vibration amplitude of the harvester. As an example, a vibration source with a frequency range of $70-110 \mathrm{~Hz}$ and an amplitude of $0.25 \mu \mathrm{m}$ is considered. According to the design considerations, the first step will be to choose geometric properties of the harvester to match the maximum value of the excitation frequency $(110 \mathrm{~Hz})$. Like any other design problem, there is no unique solution. Table 3.2 gives one possibility. In order to choose the geometrical parameters, care has been taken to select reasonable dimensions. The dimensions that are used here are similar to those used in recent experimental and theoretical research papers $[95,96]$

Table 3.1 Geometrical properties of the microbeam and piezoelectric layers

\begin{tabular}{ll}
\hline Design Variable & Value \\
\hline Length $(L)$ & $4600 \mu \mathrm{m}$ \\
Width $(b)$ & $2000 \mu \mathrm{m}$ \\
Thickness $\left(h_{\tilde{s}}\right)$ & $3.005 \mu \mathrm{m}$ \\
Thickness $\left(h_{\tilde{p}}\right)$ & $2 \mu \mathrm{m}$ \\
Tip mass $\left(M_{t}\right)$ & $2.8108 \mathrm{e}-07$ \\
\hline
\end{tabular}

The second step in designing the harvester is to find the optimal value of the resistance when the harvester works at $\Omega_{\max }$. As shown in Figure 3.11a, without any electrostatic force the optimal resistance is $786 \mathrm{k} \Omega$. In the third step, by analysing the vibration response of the harvester, the minimum initial gaps between electrodes can be determined to avoid any contact between the microbeam and the electrodes. Based on this analysis, the initial gap between the electrodes is $165 \mu \mathrm{m}$ (see Figure 3.11b). 


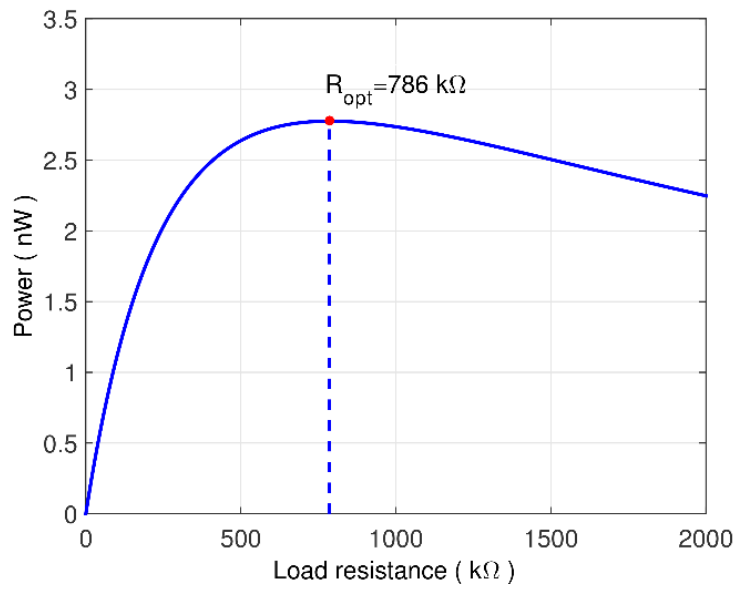

(a)

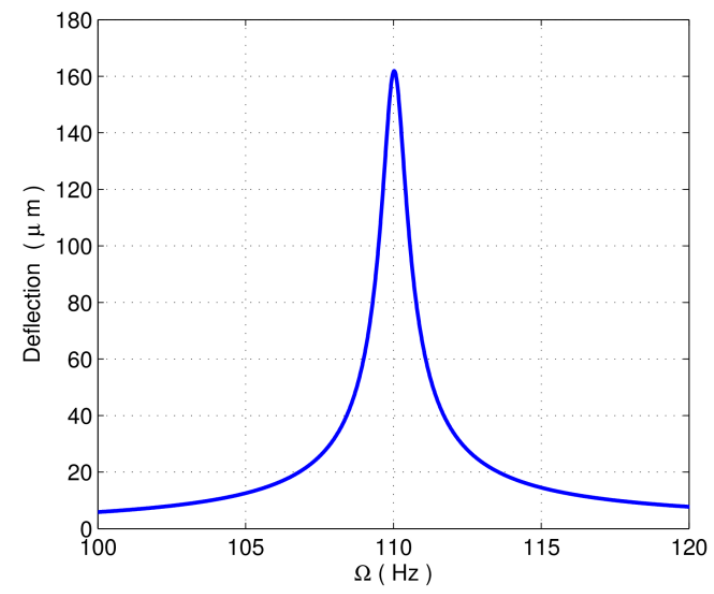

(b)

Figure 3.11 (a) Variation of the piezoelectric peak power with load resistance for a linear harvester (b) Displacement frequency response curve considering optimal resistance

In order to change the resonance frequency of the harvester, there are two possibilities: using only the variable voltage source or simultaneously varying the load resistance and the voltages source. In the first case, the harvester is designed based on a constant load resistance. As shown in Figure 3.12, by considering the optimal value of the resistance at $\Omega_{\text {max }}$ as a constant load resistance, the frequency range can be covered by changing the applied DC voltage from zero up to $50.5 \mathrm{~V}$. The pull-in voltage of the microbeam is greater than $50.5 \mathrm{~V}$ and the clearance between the electrodes is considered in the second step. 


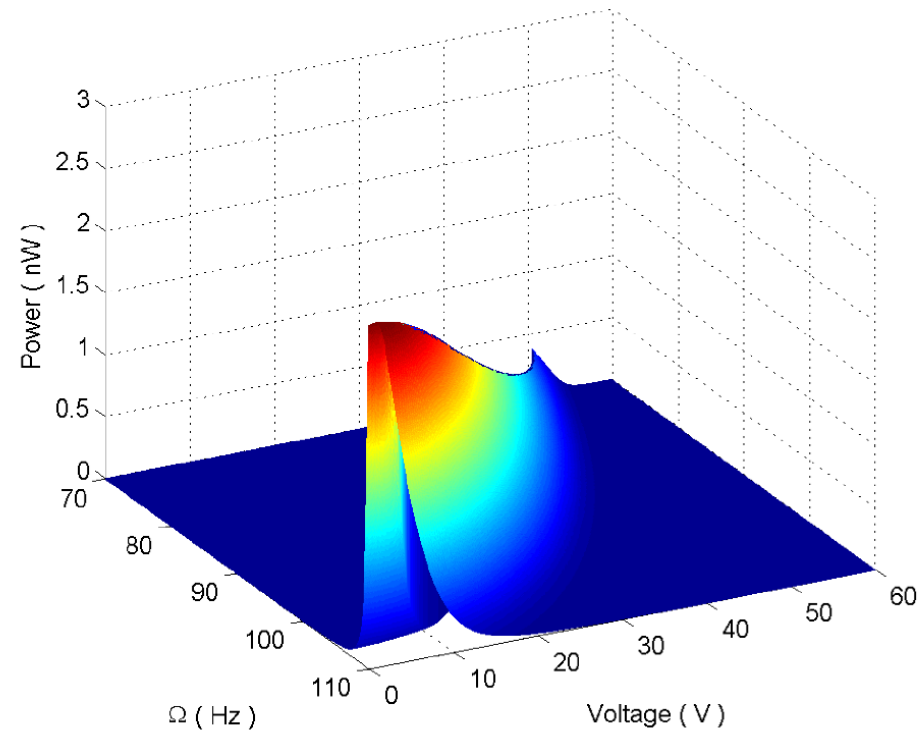

Figure 3.12 Variation of the piezoelectric peak power with DC voltage and excitation

$$
\text { frequency }\left(L_{e}=0.5 \mathrm{~L}\right)
$$

According to Figure 3.6b, by decreasing the frequency of excitation, the optimal resistance value increases. On the other hand, based on Figure $3.7 \mathrm{~b}$, increasing the value of load resistance increases the softening effect and this can be considered a second frequency adjusting parameter. Therefore, in the second case, the harvester is designed based on finding the optimal load resistance at each frequency of excitation. Figure 3.13 shows the harvested power by considering the variable load resistance and voltage source at 70 and $80 \mathrm{~Hz}$. Choosing suitable values for the load resistance and the applied DC voltage can increase the harvested power. At $70 \mathrm{~Hz}$, the harvested power can be maximized by applying $43.6 \mathrm{~V}$ and using $1.9 \mathrm{M} \Omega$ load resistance. However, in order to increase the harvested power at $80 \mathrm{~Hz}$, the tuning parameters can be considered as $V_{D C}=$ $35.3 \mathrm{~V}$ and $R=1.5 \mathrm{M} \Omega$. 


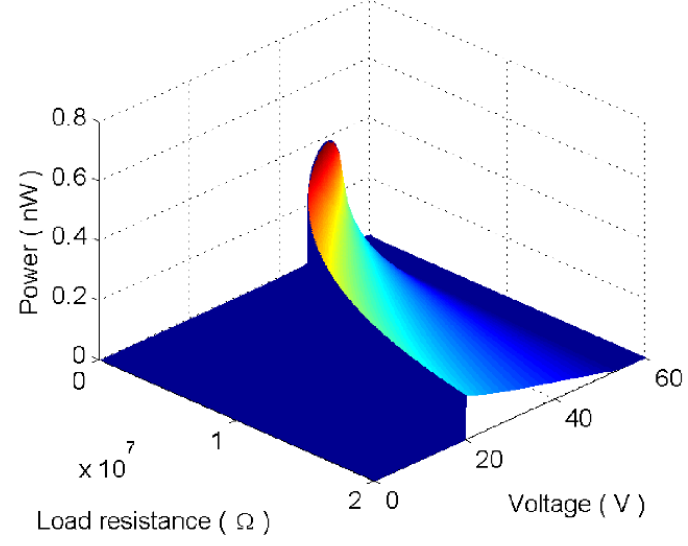

(a) $70 \mathrm{~Hz}$

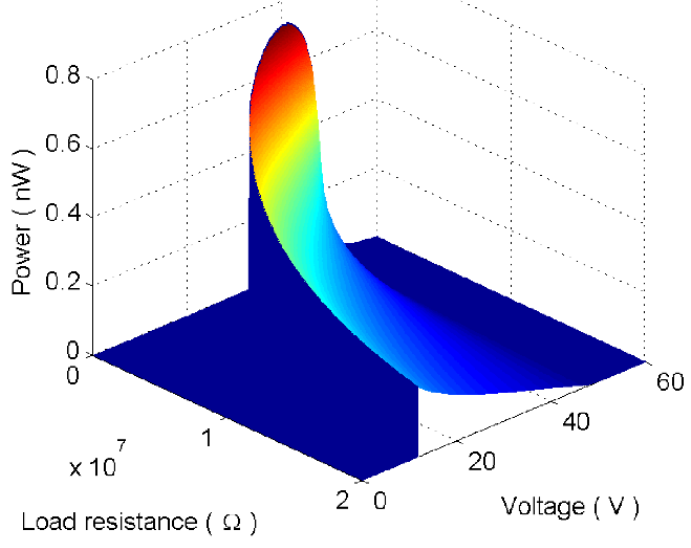

(b) $80 \mathrm{~Hz}$

Figure 3.13 Variation of the piezoelectric peak power by changing load resistance and applied voltage at 70 and $80 \mathrm{~Hz}$

By comparing the results of these two cases, the harvested power can be increased by using variable resistance and the difference is more obvious when the operational frequency range of the harvester is increased (see Figure 3.14a). As shown in Figure $3.14 \mathrm{~b}$, using variable resistance decreases the range of voltage source required to cover the given frequency range. In general, the design process can be divided into four steps which are shown in Figure 3.15.

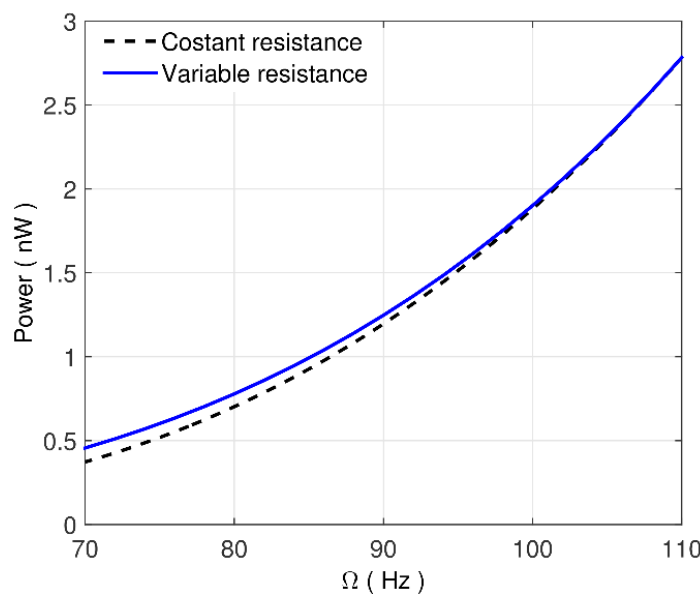

(a)

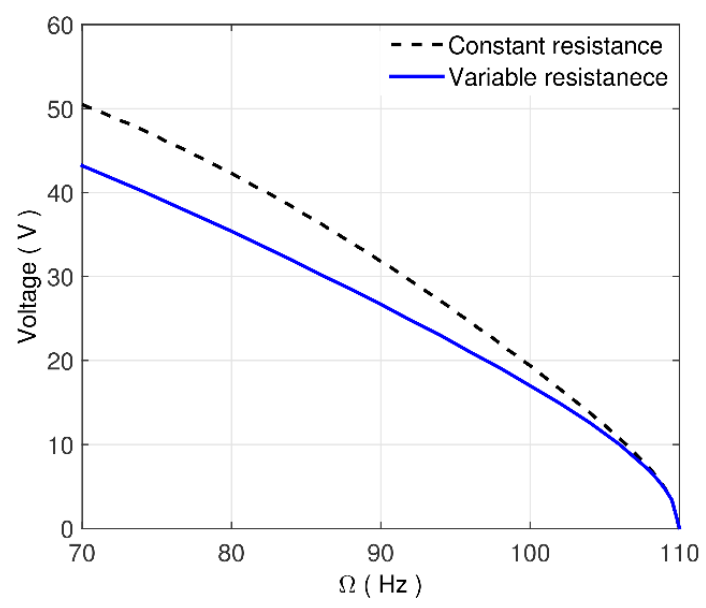

(b) 


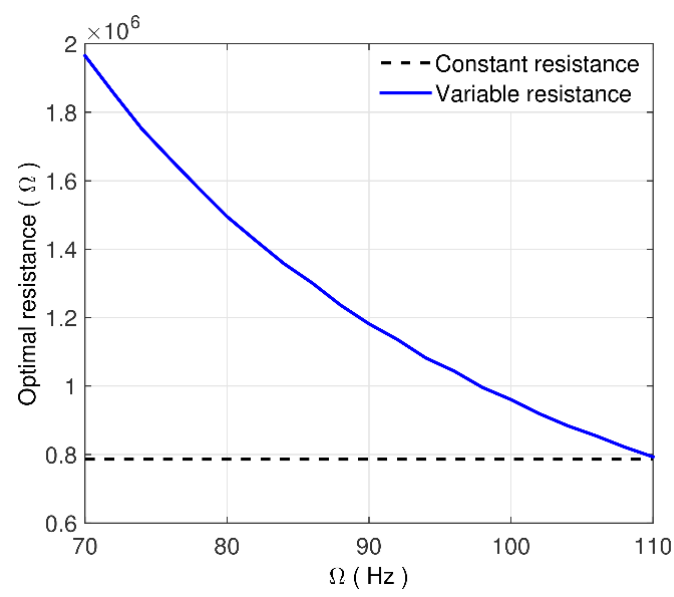

(c)

Figure 3.14 (a) Frequency range of the harvester based on increasing DC voltage (b) Voltage range for covering the frequency range (c) optimal resistance at each frequency

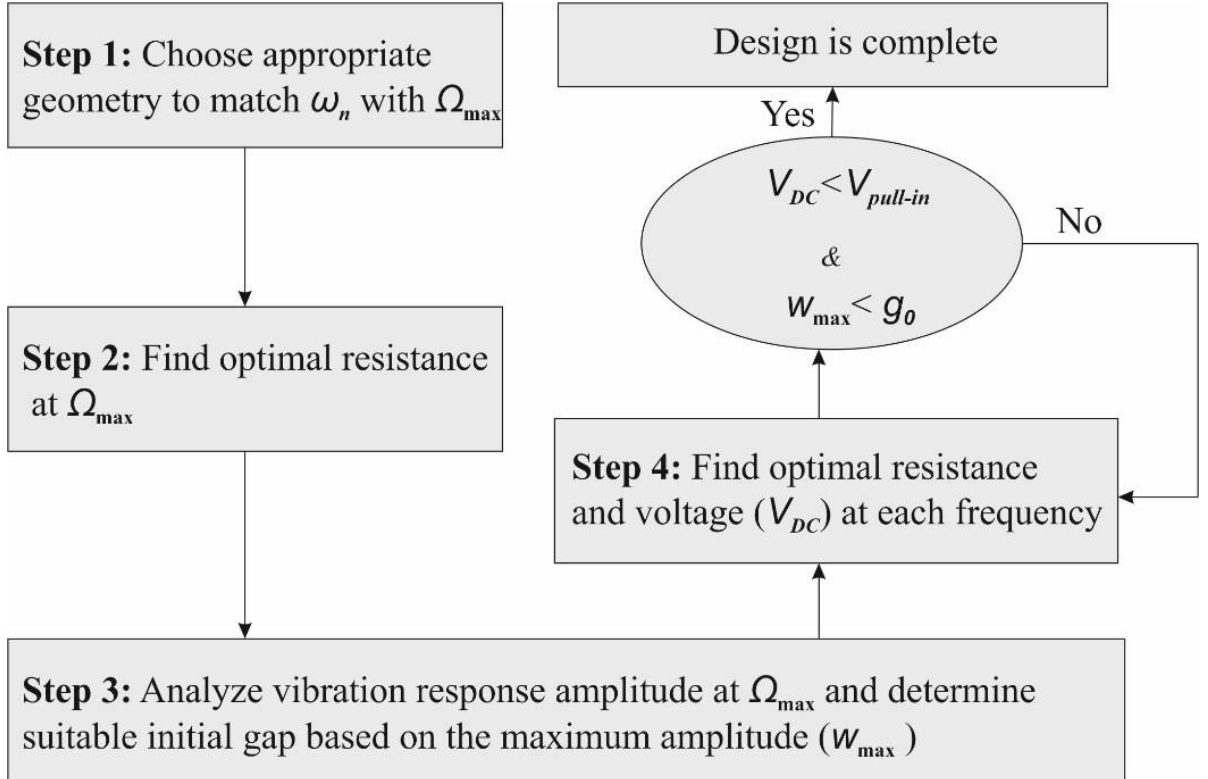

Figure 3.15 Design flow chart 


\subsection{Closure}

In this chapter, the design of a MEMS piezoelectric harvester, capable of adjusting its resonance frequency to the excitation frequency has been studied. The steady-state solution was obtained by using the harmonic balance method and the results were verified numerically. It was observed that the analytical solution can lead to significant savings in computational time, particularly when there is a need for multiple runs, when performing parametric studies for design purposes for example. Although the analytical solution requires an approximation to the electrostatic force by using a truncated Taylor's series, a convergence study can determine the number of terms that have to be retained in order to maintain sufficient accuracy. The results showed that the operating frequency bandwidth of the harvester can be increased by using a variable DC voltage source. In addition, due to the strong coupling between electrical and mechanical equations, the results showed that load resistance can be considered a second adjusting frequency parameter. A MEMS piezoelectric harvester was designed for a vibration source with a frequency range of $70-110 \mathrm{~Hz}$ and $0.25 \mu \mathrm{m}$ amplitude of base excitation. The disadvantage of the proposed system is the effect of the softening nonlinearity of the electrostatic part of the harvester which results in a lower level of harvested energy. In addition, due to manufacturing uncertainties sometimes there is a need to tune the harvester's resonance frequency to a higher frequency, which is behind the capability of the model proposed in this chapter. In order to tackle this problem, the next chapter includes devising a mechanism that has a hardening effect on the system and hence the frequency of the harvester can be adjusted on both sides of its linear resonance frequency. 


\section{Chapter 4: Effects of manufacturing uncertainties in MEMS Energy Harvesters}

This chapter provides an introduction to Uncertainty Analysis (UA) in general and particularly in MEMS devices. A brief description of different methods for uncertainty representation is given and then two main concepts in UA, namely representation and propagation are discussed. The focus is then placed on the different types of uncertain parameters in designing a MEMS piezoelectric harvester. Afterwards, an electrostatic device is proposed to improve the performance of MEMS piezoelectric harvesters in the presence of manufacturing uncertainties. In order to show the results, an experimentally measured statistical properties available in the literature is considered, and Monte Carlo Simulation (MCS) is used for uncertainty propagation.

\subsection{Introduction to Uncertainty Analysis}

Nowadays, real-world structures are subject to uncertainties arising from material imperfections, machining tolerance, and manufacturing processes. Using deterministic models based on nominal parameters might over- or under-estimate the behaviour of the actual structures. Therefore, it is necessary to create statistic models which can be more reliable by providing additional information.

Generally, there are two types of uncertainties: epistemic and aleatoric (irreducible and inherent) [97]. Epistemic uncertainty is also known as reducible uncertainty, subjective uncertainty, and model form uncertainty. There are two types of epistemic uncertainty: the first type is uncertainty which is related to a model itself and can be reduced by increasing knowledge about the system or the surrounding environment. The second type of epistemic uncertainty is uncertainty in model parameters. For example, uncertainty in the estimation of the relevant parameters can increase uncertainty in the model (parametric epistemic uncertainty). Parametric epistemic uncertainty can be reduced by increasing the number of measurements, or by using more relevant data to estimate the 
parameters. On the other hand, aleatory uncertainty is induced by inherent randomness. There are two types of aleatory uncertainty: variable aleatory uncertainty and physical aleatory uncertainty [98]. All variations in structural parameters due to the accumulation of manufacturing tolerances or environmental erosion are considered as variable aleatory uncertainty, which is the main concern of this chapter. However, physical aleatory uncertainty is caused by physical phenomena and remains the same even if all the stochastic variables of the problem were replaced by deterministic values. The different types of uncertainty have been summarized in Figure 4.1.

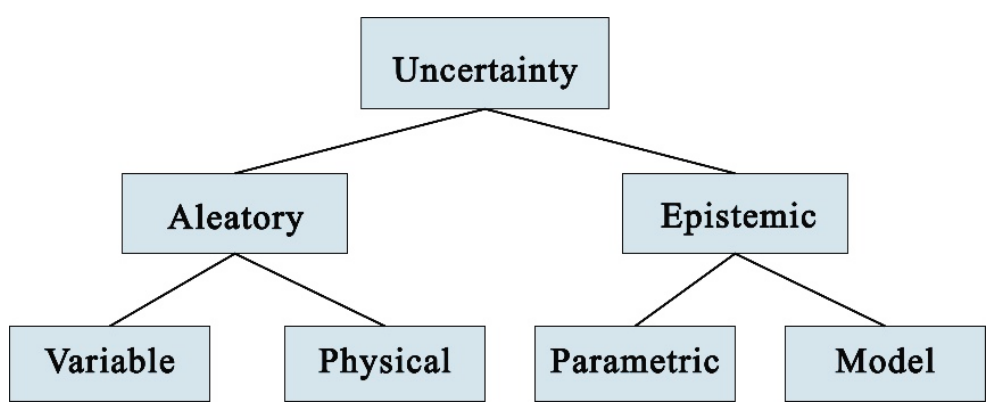

Figure 4.1 Aleatory and epistemic uncertainty

\subsection{Representation and Propagation of Uncertainty}

In order to deal with uncertainty in the field of structural dynamics, there are two main concerns: namely representation and propagation which are discussed in the following subsections.

\subsubsection{Representation}

Uncertainty can be modelled by different frameworks, which may be categorised into two groups: probabilistic models and non-probabilistic models. There are many probabilistic models such as classical probability theory (or random parameters), random fields, Bayesian, Evidence (Dempster-Shafer theory (DST)) and Possibility theory. For example, in probability theory, a domain of possible values for the random parameter $\mathrm{X}$ is defined and the frequency of occurrences or likelihood of the random parameter being inside a certain domain is given by a Probability Density Function (PDF). The PDF can be used to evaluate the probability of occurrence of a random parameter in a particular domain of interest [51]. Random fields theory is used to model the spatial variation of 
uncertain parameters over a region in which the variation takes place. Alternatively, there are some methods based on the non-probabilistic approach such as Interval model and Fuzzy sets. In the Interval approach, the uncertain variables can be variable within intervals between extreme values and no assumption is made about the probability distribution of the uncertain variables.

The Fuzzy logic is one of the elder statesmen of contenders within probability theory, which models uncertainty based on indistinctive definition (instead of probability distribution). In Fuzzy Set theory, an element x may be associated (with given weight) with a number of different sets. Initially, the most intensive use of Fuzzy logic in engineering was associated with control, however in recent years Fuzzy Sets have been used a great deal to construct data-based classifiers for condition monitoring and structural health monitoring applications [53].

\subsubsection{Uncertainty Propagation}

There are different uncertainty propagation methods such as the Monte Carlo Simulation (MCS) method, the asymptotic integral method, and the first and second order perturbation methods. Monte Carlo Simulation (MCS) is the most accurate and reliable propagation method, which is explained in this section. MCS is based on a sampling method and has been frequently used in the literature for the purposes of uncertainty propagation.

In MCS, a large number of samples of uncertain parameters is generated according to the PDF of parameter, while the respective response values are evaluated from deterministic analysis. The mean and covariance matrix of the output vector from the analytical/numerical model can then be directly evaluated from the scatter of responses and the system parameters that provide the input to the simulation [97].

The generation of samples in the Monte-Carlo process can be carried out via different methods such as multivariate normal sampling [99], Latin Hypercube Sampling (LHS) and Orthogonal Array Sampling [100]. Multivariate normal sampling and LHS have been used in this thesis. Generally, this method is used when the uncertain parameters belong to a multivariate normal distribution. In cases where the uncertain parameters are uncorrelated; the covariance matrix is diagonal. Therefore, the sampling is straightforward as the samples from each component of the random vector can be taken independently to generate a number of sample vectors of the random vector. It is 
noteworthy that once the uncertain parameters are correlated, the procedure will be slightly different.

\subsection{Manufacturing uncertainties in MEMS devices}

Demands for innovative MEMS devices has led to engineers developing micro fabrication techniques. Generally, MEMS are manufactured based on two lithographybased methods: bulk and surface micromachining [101]. Bulk micromachining is the earliest and best-characterized method of producing micromachined devices. It mainly consists of wet etching of silicon wafer, which is the most common substrate for micromachined structures. Unlike bulk micromachining, surface micromachining builds microstructures by deposition and etching different structural layers over a substrate. In addition, there are other processes such as mask alignment, chemical polishing and drying which need to be carried out in the fabrication of MEMS devices. Therefore, the MEMS fabrication processes do not guarantee infinite repeatability of the mechanical or geometrical properties of final fabricated devices. Generally, manufacturing tolerances of MEMS devices are high and in some cases, they can be higher than $\pm 10 \%$ of nominal values [102]. This fact should be taken into account while designing MEMS, because it can significantly affect the performance of the devices.

Uncertainty analysis of MEMS devices has been studied by several authors. Gurav et al. [103] investigated design optimization under uncertainty for micro energy harvesters. They considered both uncertainties in geometric parameters and material properties, and showed that accounting for uncertainties would sacrifice $20 \%$ of the power output compared to deterministic optimization results. Shanmugavalli et al. [104] illus trated the use of interval methods to study the electromechanical behaviour of MEMS in the presence of manufacturing and process uncertainties. They achieved a robust and reliable analysis of pull-in voltage of fixed-fixed beam in a most efficient way. Agarwal and Aluru [105] presented a framework to quantify different kinds of outputs in MEMS structures such as deformation and electrostatic pressure in these devices. The same researchers [106] proposed a framework to include the effect of uncertain design parameters in MEMS devices. Based on this framework, they investigated the effect of variations in Young's modulus, induced because of variations in the manufacturing process parameters or heterogeneous measurements, on the performance of a MEMS 
switch. Alexeenko et al. [102] studied the effects of uncertainties in gas damping models, geometry, and mechanical properties on the dynamics of MEMS capacitive switch. They showed that due to the parameter uncertainties, the nominal switch, i.e. the switch with the average properties, does not actuate at the mean actuation voltage. Additionally, device-to-device variability leads to significant differences in dynamics.

Based on the experimental results [102], the most important variation of parameters in the fabrication of MEMS devices include thickness, air gap and Young's modulus. These variations can decrease the performance of the harvester significantly. In the next section, an electrostatic device is proposed to compensate the adverse effect of manufacturing uncertainties on the performance of MEMS piezoelectric harvesters.

\subsection{Model description and mathematical modelling}

Figure 4.2 shows the model proposed in this chapter. The model is an isotropic microbeam of length $\boldsymbol{L}$, width $\boldsymbol{b}$, thickness $\boldsymbol{h}$, density $\boldsymbol{\rho}_{\tilde{\boldsymbol{s}}}$ and Young's modulus $\boldsymbol{E}_{\tilde{\boldsymbol{s}}}$, sandwiched with piezoceramic layers having length $\boldsymbol{L}_{\boldsymbol{c}}$, thickness $\boldsymbol{h}_{\tilde{\boldsymbol{p}}}$, Young's modulus $\boldsymbol{c}_{\mathbf{1 1}}^{\boldsymbol{E}}$ and density $\boldsymbol{\rho}_{\widetilde{\boldsymbol{p}}}$ throughout the micro-beam length and located between two straight-shaped electrodes and one arc-shaped electrode. As illustrated in Figure 4.2, the piezoceramic layers are connected to the resistance $(\boldsymbol{R})$, and the coordinate system is attached to the middle of the left end of the micro-beam, where $x$ and $z$ refer to the horizontal and vertical coordinates respectively. The free end of the micro-beam is attached to two arc-shaped comb fingers, which subtend angle $\boldsymbol{\alpha}$ at the base of the beam and remain parallel to the fixed arc-shaped electrode. The governing equation of transverse motion can be written as

$$
\begin{gathered}
\frac{\partial^{2}}{\partial \hat{x}^{2}}\left(E I\left(\hat{x}, \hat{\psi}_{1}\right) \frac{\partial^{2} \widehat{w}_{r e l}^{p}}{\partial \hat{x}^{2}}\right)+m\left(\hat{x}, \widehat{\psi}_{2}\right) \frac{\partial^{2} \widehat{w}_{r e l}^{p}}{\partial \hat{t}^{2}}+\hat{c}_{a} \frac{\partial \widehat{w}_{r e l}^{p}}{\partial \hat{t}} \\
+F_{f}\left(\hat{\psi}_{3}\right) \frac{\partial^{2} \widehat{w}_{r e l}^{p}}{\partial \hat{x}^{2}}-\hat{\vartheta}_{p}\left(\hat{\psi}_{4}\right) v_{p}(\hat{t})\left(\frac{d \delta(\hat{x})}{d \hat{x}}-\frac{d \delta\left(\hat{x}-L_{c}\right)}{d \hat{x}}\right) \\
=F_{e}\left(\hat{\psi}_{5}\right)+z_{0} \widehat{\Omega}^{2}\left(m\left(\hat{x}, \hat{\psi}_{2}\right)+M_{t} \delta(\hat{x}-L)\right) \cos (\hat{\Omega} \hat{t})
\end{gathered}
$$

and subject to the following boundary conditions 


$$
\begin{gathered}
\widehat{w}_{r e l}^{p}(0, \hat{t})=0 \quad \frac{\partial \widehat{w}_{r e l}^{p}(0, \hat{t})}{\partial \hat{x}}=0 \\
\frac{\partial}{\partial \hat{x}}\left(E I\left(\widehat{x}, \widehat{\psi}_{1}\right) \frac{\partial^{2} \widehat{w}_{r e l}^{p}(L, \hat{t})}{\partial \hat{x}^{2}}\right)=M_{t}\left(\frac{\partial^{2} \widehat{w}_{r e l}^{p}(L, \hat{t})}{\partial \hat{t}^{2}}\right) \\
\frac{\partial^{2} \widehat{w}_{r e l}^{p}(L, \hat{t})}{\partial \hat{x}^{2}}=0
\end{gathered}
$$

where $\hat{\psi}_{1}, \hat{\psi}_{2}, \ldots, \hat{\psi}_{n}$ shows the stochastic parameters, which have been used as an input to the mathematical model.

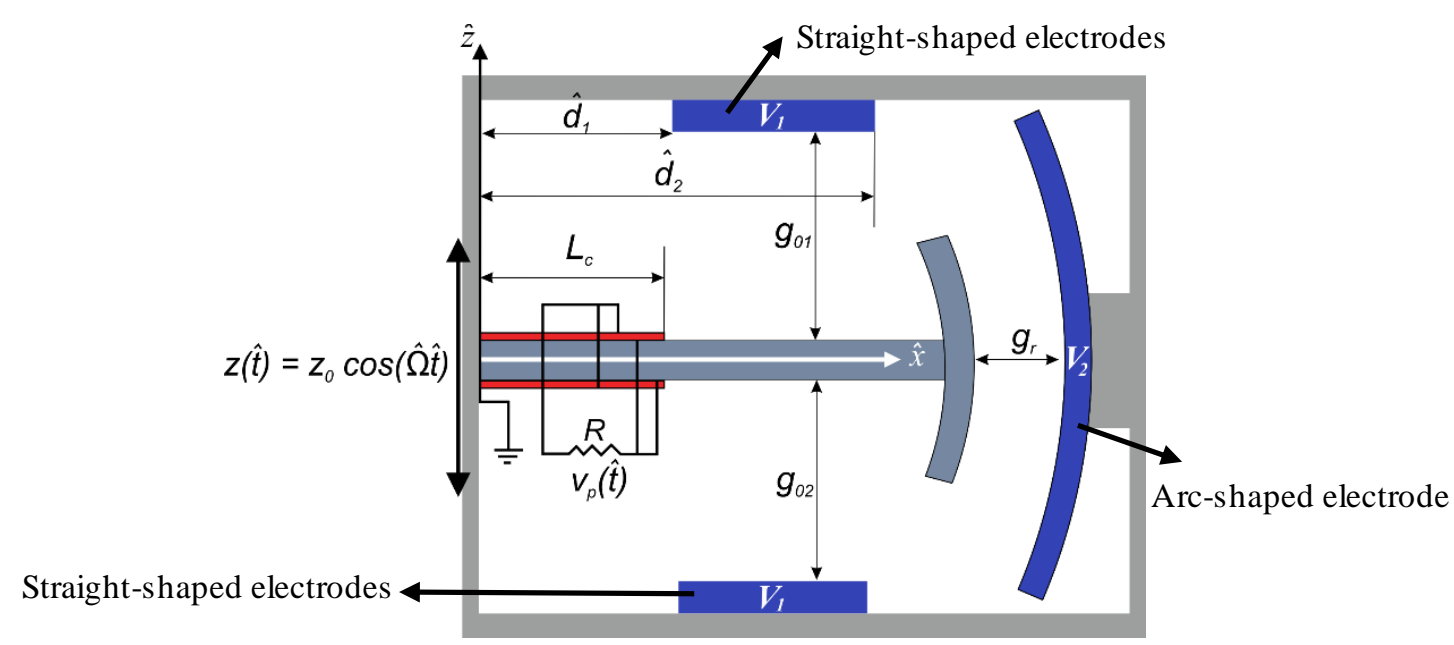

Figure 4.2 Schematic of the proposed energy harvester

In equation (4.1), $\widehat{w}_{r e l}^{p}$ is the transverse deflection of the beam relative to its base at position $\hat{x}$ and time $\hat{t}, c_{a}$ is the viscous air damping coefficient, $\delta(\hat{x})$ is the Dirac delta function, $z(\hat{t})$ is the base excitation function, $F_{f}$ is the follower force which is applied to the harvester by the arc-shaped electrode, $F_{e}$ is the electrostatic force which is applied to the harvester by the straight-shaped electrodes, $v_{p}(\hat{t})$ is the voltage across the electrodes of each piezoceramic layer, $\hat{\vartheta}_{p}$ is the coupling term. Using Kirchhoff's laws, the electrical circuit equation can be expressed by

$$
C_{\tilde{p}}\left(\hat{\psi}_{6}\right) \frac{d v_{p}(\hat{t})}{d \hat{t}}+\frac{v_{p}(\hat{t})}{2 R}+i_{\tilde{p}}^{p}\left(\hat{t}, \hat{\psi}_{7}\right)=0
$$

where the internal capacitance $\left(C_{\tilde{p}}\right)$, coupling term $\left(\hat{\vartheta}_{p}\right)$ and the current source $\left(i_{\tilde{p}}^{p}\right)$ can be obtained as 


$$
\begin{gathered}
C_{\tilde{p}}\left(\hat{\psi}_{6}\right)=\frac{\bar{\varepsilon}_{33}^{s} b L_{c}}{h_{\tilde{p}}}, \quad \hat{\vartheta}_{p}\left(\hat{\psi}_{4}\right)=\frac{\bar{e}_{31} b}{h_{\tilde{p}}}\left(\left(h_{\tilde{p}}+\frac{h_{\tilde{s}}}{2}\right)^{2}-\frac{h_{\tilde{s}}^{2}}{4}\right), \\
i_{\tilde{p}}^{p}\left(\hat{t}, \hat{\psi}_{7}\right)=\frac{\bar{e}_{31} b}{2}\left(h_{\tilde{p}}+h_{\tilde{s}}\right) \int_{0}^{L_{c}} \frac{\partial^{3} \widehat{w}_{r e l}^{p}(\hat{x}, \hat{t})}{\partial \hat{x}^{2} \partial \hat{t}} d \hat{x}
\end{gathered}
$$

and $\bar{\varepsilon}_{33}^{S}$ is the permittivity component at constant strain with the plane stress assumption for the beam. Using electrostatic principles, the electrostatic force between the microbeam and the straight electrodes $\left(F_{e}\right)$ can be written as [93]

$$
F_{e}\left(\hat{\psi}_{5}\right)=\frac{\varepsilon_{0} b H(\hat{x})}{2}\left(\frac{V_{1}^{2}}{\left(g_{01}-\widehat{w}_{r e l}^{p}\right)^{2}}-\frac{V_{1}^{2}}{\left(g_{02}+\widehat{w}_{r e l}^{p}\right)^{2}}\right)
$$

where

$$
H(\hat{x})=H\left(\hat{x}-\hat{d}_{1}\right)-H\left(\hat{x}-\hat{d}_{2}\right)
$$

In equation (4.5), $\varepsilon_{0}$ is the permittivity of free space, $H(\hat{x})$ is the Heaviside function, $V_{1}$ is the DC voltage applied to the straight electrodes, $g_{01}$ and $g_{02}$ are the air gaps between the micro-beam and the straight electrodes. By applying voltage to the arc-shaped electrode shown in Figure 4.2, the amplitude of $F_{f}$ can be tuned. Figure 4.3 shows that for a small angular deflection of the micro-beam $(\theta)$, the angular overlap between the finger and the arc-shaped electrode is always $2 \alpha$ and the force remains a follower force in all conditions.

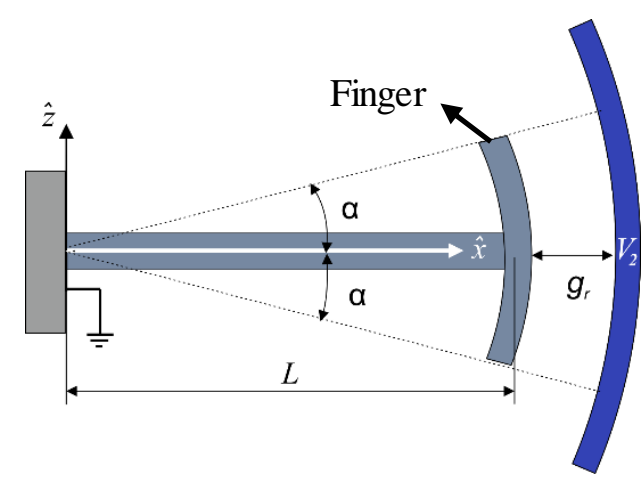

(a)

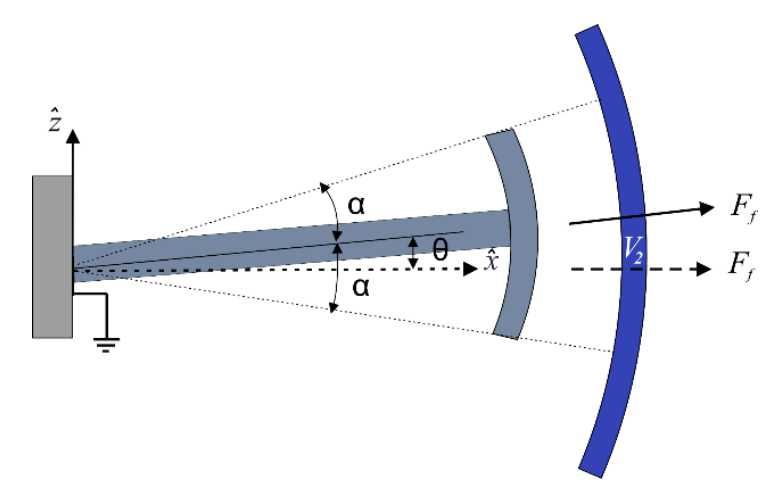

(b)

Figure 4.3 A micro-beam with an arc-shaped comb finger in its (a) unperturbed state and (b) perturbed state. 
Based on electrostatic principles, the amplitude of the follower force $\left(F_{f}\right)$ can be written as

$$
F_{f}\left(\widehat{\psi}_{3}\right)=\frac{\varepsilon_{0} b}{g_{r}^{2}} V_{2}^{2} L \alpha
$$

where $g_{r}$ is the radial gap between the comb fingers and arc-shaped electrode, $b$ is the width of the resonator, $L$ is the length of the beam (the thickness of the arc-shaped electrode can be ignored), and $V_{2}$ is the voltage applied to the arc-shaped electrode. For convenience, we introduce the following non-dimensional variables

$$
w=\frac{\widehat{w}}{g_{01}}, \quad x=\frac{\hat{x}}{L}, \quad t=\hat{t} \sqrt{\frac{E I_{C}}{m_{C} L^{4}}}, \quad \Omega=\hat{\Omega} \sqrt{\frac{m_{C} L^{4}}{E I_{C}}}, \quad l_{c}=\frac{L_{c}}{L}
$$

where $E I_{C}$ and $m_{C}$ are related to the bending stiffness and mass per unit length of the beam with piezoelectric layers. Substituting equation (4.8) into equations (4.1) and (4.3), the following non-dimensional equations are obtained

$$
\begin{gathered}
\frac{\partial^{2}}{\partial x^{2}}\left(s_{1}\left(x, \psi_{1}\right) \frac{\partial^{2} w_{r e l}^{p}}{\partial x^{2}}\right)+s_{2}\left(x, \psi_{2}\right) \frac{\partial^{2} w_{r e l}^{p}}{\partial t^{2}}+c_{a} \frac{\partial w_{r e l}^{p}}{\partial t}+\alpha_{f}\left(\psi_{3}\right) V_{2}^{2} \frac{\partial^{2} w_{r e l}^{p}}{\partial x^{2}} \\
-\vartheta_{p}\left(\psi_{4}\right) v_{p}(t)\left(\frac{d \delta(x)}{d x}-\frac{d \delta\left(x-l_{c}\right)}{d x}\right) \\
=\alpha_{e}\left(\psi_{5}\right) V_{1}^{2}\left(\frac{H(x)}{\left(1-w_{r e l}^{p}\right)^{2}}-\frac{H(x)}{\left(r\left(\psi_{6}\right)+w_{r e l}^{p}\right)^{2}}\right) \\
+\left(\sigma_{1} s_{2}\left(x, \psi_{2}\right)+\sigma_{2} \delta(x-1)\right) \Omega^{2} \cos (\Omega t) \\
\frac{d v_{p}(t)}{d t}+\lambda\left(\psi_{7}\right) v_{p}(t)+\gamma\left(\psi_{8}\right) \int_{0}^{l_{c}} \frac{\partial^{3} w_{r e l}^{p}}{\partial x^{2} \partial t} d x=0
\end{gathered}
$$

where the coefficients of equation (4.9) and (4.10) have been given in Appendix A. Using the Galerkin decomposition method and considering a single-mode approximation, equations (4.9) and (4.10) can be converted into a system of differential equations 


$$
\begin{gathered}
\ddot{U}(t)+2 \mu \omega_{n} \dot{U}(t)+\omega_{n}^{2} U(t)-\theta_{p} v_{p}(t) \\
=\alpha_{t} \int_{0}^{1}\left(\frac{V_{1}^{2} H(x)}{(1-U \varphi(x))^{2}}-\frac{V_{1}^{2} H(x)}{(r+U \varphi(x))^{2}}\right) \varphi d x \\
\quad+F \Omega^{2} \cos (\Omega t) \\
\dot{v}_{p}(t)+\lambda v_{p}(t)+\beta \dot{U}(t)=0
\end{gathered}
$$

where $\varphi(x)$ is the ith linear undamped mode shape of the straight micro-beam and $U(t)$ is the ith generalized coordinate. The coefficients of equations (4.10) and (4.11) have been introduced in Appendix A. Due to the electrostatic nonlinearity in equation (4.10), finding an analytical solution to study the dynamic behaviour of the system is quite complicated. However, there are different methods to find an approximate analytical solution to equations (4.10) and (4.11). Previously in Chapter 3 we used the harmonic balance method to study the dynamic behaviour of the system by considering an approximate electrostatic force using Taylor expansion. Based on the assumption of symmetrical electrostatic force in the approximation, it was shown that acceptable convergence can be obtained by including terms up to ninth-order. However, in the presence of manufacturing uncertainties, the electrostatic force could be unsymmetrical due to variabilities in the air gap, and more terms may need to be included to reach acceptable convergence. Therefore, using the harmonic balance method makes uncertainty propagation tedious because for every different sample, the number of truncated terms should be determined. In this chapter, the shooting method [107] is used to investigate the dynamic behaviour of the system. Generally, the shooting method is a powerful and useful method to find periodic solutions to a nonlinear system, and it is computationally more time efficient than direct integration methods. The shooting method can also find unstable solutions, although this is not needed for the analysis undertaken here. By introducing $X_{1}=U, X_{2}=\dot{U}$ and $X_{3}=v_{p}$, equations (4.10) and (4.11) can be rewritten as 


$$
\begin{gathered}
\dot{X}_{1}=X_{2} \\
\dot{X}_{2}=F \cos (\Omega t)-2 \mu \omega_{n} X_{2}-\omega_{n}^{2} X_{1}+\theta_{p} X_{3} \\
+\alpha_{t} V_{1}^{2} \int_{0}^{1}\left(\frac{H(x)}{\left(1-X_{1} \varphi\right)^{2}}-\frac{H(x)}{\left(r+X_{1} \varphi\right)^{2}}\right) \varphi d x \\
\dot{X}_{3}=-\lambda X_{3}-\beta X_{2}
\end{gathered}
$$

To find a periodic solution to equations (4.12), (4.13) and (4.14), an appropriate set of initial conditions $\left(\eta_{1}, \eta_{2}, \eta_{3}\right)$ must be identified. To proceed with the shooting technique, for convenience, the following variables are defined:

$$
\begin{array}{ccc}
X_{4}=\frac{\partial X_{1}}{\partial \eta_{1}}, & X_{5}=\frac{\partial X_{1}}{\partial \eta_{2}}, & X_{6}=\frac{\partial X_{1}}{\partial \eta_{3}} \\
X_{7}=\frac{\partial X_{2}}{\partial \eta_{1}}, & X_{8}=\frac{\partial X_{2}}{\partial \eta_{2}}, & X_{9}=\frac{\partial X_{2}}{\partial \eta_{3}} \\
X_{10}=\frac{\partial X_{3}}{\partial \eta_{1}}, & X_{11}=\frac{\partial X_{3}}{\partial \eta_{2}}, & X_{12}=\frac{\partial X_{3}}{\partial \eta_{3}}
\end{array}
$$

The shooting technique requires simultaneously integrating equations (4.12), (4.13) and (4.14), plus the time derivatives of the variables $\left(\dot{X}_{4}, \ldots, \dot{X}_{12}\right)$ in the time domain for one period of excitation. The initial conditions for solving the set of differential equations are defined as

$$
\begin{array}{cccc}
X_{1}(0)=\eta_{10}, & X_{2}(0)=\eta_{20}, & X_{3}(0)=\eta_{30}, & X_{4}(0)=1, \\
X_{5}(0)=0, & X_{6}(0)=0, & X_{7}(0)=0, & X_{8}(0)=1, \\
X_{9}(0)=0, & X_{10}(0)=0, & X_{11}(0)=0, & X_{12}(0)=1
\end{array}
$$

$\eta_{10}, \eta_{20}$ and $\eta_{30}$ are initial guesses for the initial conditions that result in periodic solution. There is no straightforward way to find the initial conditions for the periodic solutions and we need to try different initial guesses for the initial conditions. Generally, these initial guesses deviate from the exact values by an error or correction $\delta \boldsymbol{\eta}$. By calculating the values of $X_{4}, \ldots, X_{12}$ at one-period and substituting them in the algebraic system of equations below, the error can be found for each set of initial guesses [107]. 


$$
\left[\left[\begin{array}{ccc}
X_{4} & X_{5} & X_{6} \\
X_{7} & X_{8} & X_{9} \\
X_{10} & X_{11} & X_{12}
\end{array}\right]-[I]\right]\left[\begin{array}{l}
\delta \eta_{1} \\
\delta \eta_{2} \\
\delta \eta_{3}
\end{array}\right]=\left[\begin{array}{l}
\eta_{10}-X_{1}\left(T, \eta_{10}, \eta_{20}, \eta_{30}\right) \\
\eta_{20}-X_{2}\left(T, \eta_{10}, \eta_{20}, \eta_{30}\right) \\
\eta_{30}-X_{3}\left(T, \eta_{10}, \eta_{20}, \eta_{30}\right)
\end{array}\right]
$$

By trying different initial guesses and using equation (4.17), the error $\left(\delta \eta_{1}, \delta \eta_{2}\right.$ and $\left.\delta \eta_{3}\right)$ can be minimized and convergence is achieved. Then, peak power through the resistance can be obtained by substituting $v_{p}$ into the following equation

$$
P_{0}=\frac{v_{p}^{2}}{R}
$$

\subsection{Numerical results and discussion}

To demonstrate the analysis presented in the previous section, a bimorph piezoelectric micro cantilever beam is considered with the same characteristics given in Table 2.1. The only difference is related to the tip mass, viscous air damping coefficient $\left(c_{a}\right)$ and the additional air gap between arc-shaped electrodes, which have been given in Table 4.1.

Table 4.1 Geometrical properties of the arch-shaped electrodes.

\begin{tabular}{lc}
\hline Design Variable & Value \\
\hline Width, $b(\mu \mathrm{m})$ & 1000 \\
Thickness of arch-shape electrode, $h_{r}(\mu \mathrm{m})$ & 5 \\
Radial air gap, $g_{r}(\mu \mathrm{m})$ & 3 \\
Viscous air damping coefficient, $c_{a}(\mathrm{~N} . \mathrm{s} / \mathrm{m})$ & 0.002
\end{tabular}

Based on the experimental results [102], the most important variations in the fabrication parameters include thickness, air gap and Young's modulus. To show the effect of these parameters on the performance of the harvester, a Gaussian distribution of parameters is assumed and given in Table 4.2.

Table 4.2 Parameters most sensitive to manufacturing uncertainties[102].

\begin{tabular}{lccc}
\hline Data & Mean & Std & COV $(\%)$ \\
\hline Thickness, $h_{\tilde{s}}(\mu \mathrm{m})$ & 4 & 0.35 & 8.75 \\
Thickness, $h_{\tilde{p}}(\mu \mathrm{m})$ & 2 & 0.175 & 8.75 \\
Young's modulus, $E_{\tilde{s}}(\mathrm{GPa})$ & 169.6 & 16.58 & 9.78 \\
Young's modulus, $E_{\tilde{p}}(\mathrm{GPa})$ & 65 & 6.35 & 9.78 \\
Air gap, $g_{0}(\mu \mathrm{m})$ & 40 & 2.52 & 6.3 \\
Air gap, $g_{r}(\mu \mathrm{m})$ & 3 & 0.18 & 6.3 \\
\hline
\end{tabular}




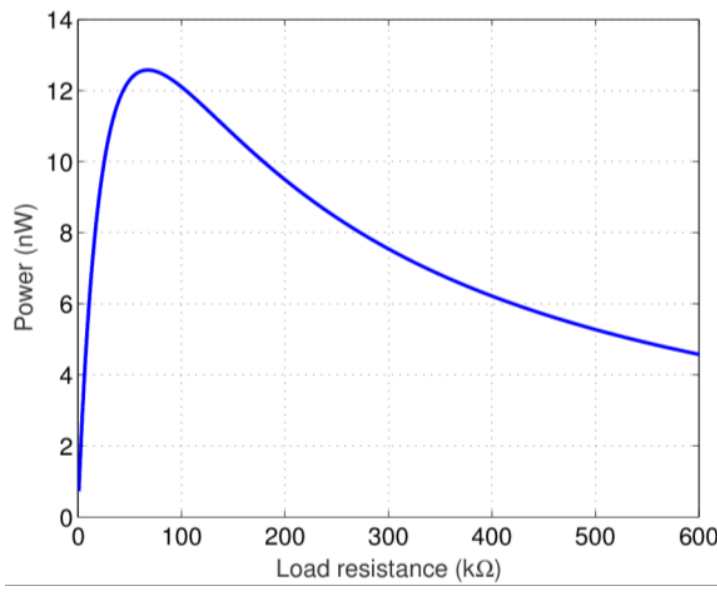

(a)

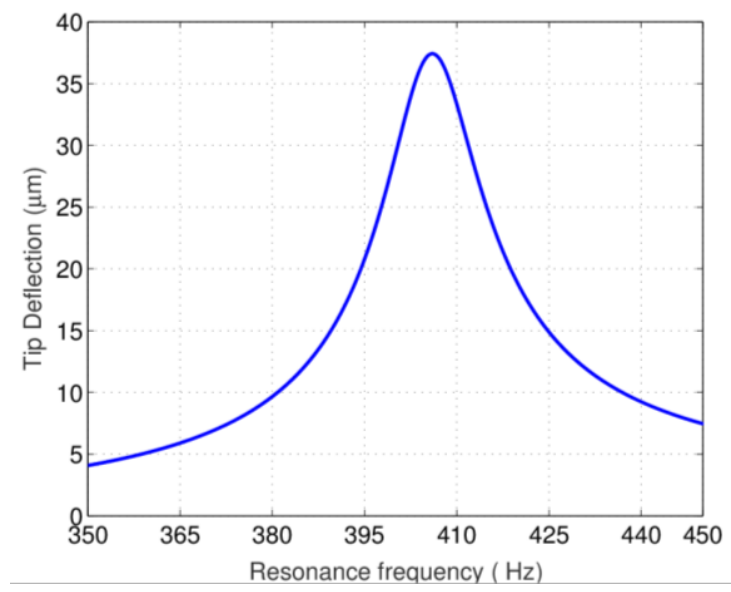

(b)

Figure 4.4 (a) Variation of the piezoelectric peak power with load resistance at nominal resonance frequency (b) Displacement frequency response curve with the optimal resistance

Considering the mean parameters of the microbeam, the optimal resistance of the harvester is obtained at its resonance frequency. As shown in Figure 4.4a, by exciting the harvester at its resonance frequency, $12.6 \mathrm{nW}$ power can be harvested at the optimal resistance. In addition, as Figure $4.4 \mathrm{~b}$ shows, the maximum deflection of the beam at resonance frequency is less than $40 \mu \mathrm{m}$. To investigate the effect of manufacturing uncertainties on the performance of the MEMS piezoelectric harvester, different numbers of samples are generated and Monte Carlo Simulation is used for uncertainty propagation. Figure 4.5a shows that the Probability Density Function (PDF) of the power does not significantly change when the number of samples is increased from 1500 to 2000 , hence 2000 samples will be enough for uncertainty analysis. 


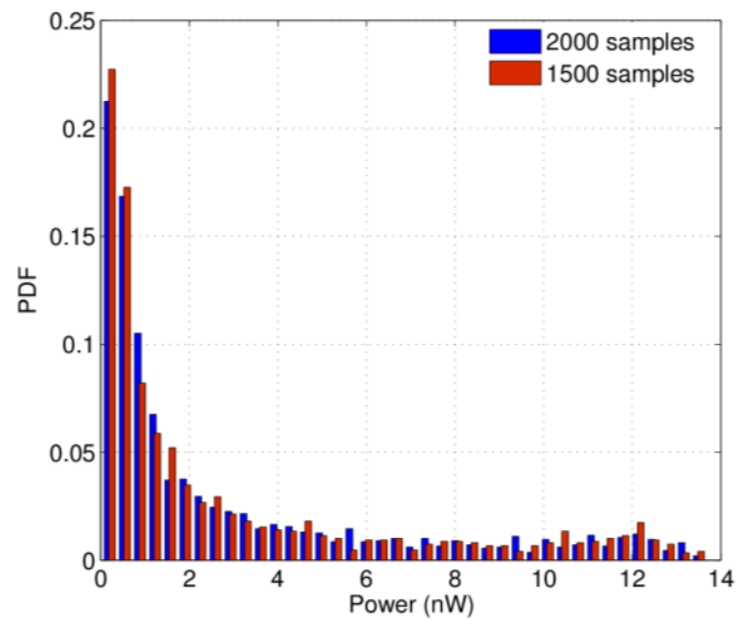

(a)

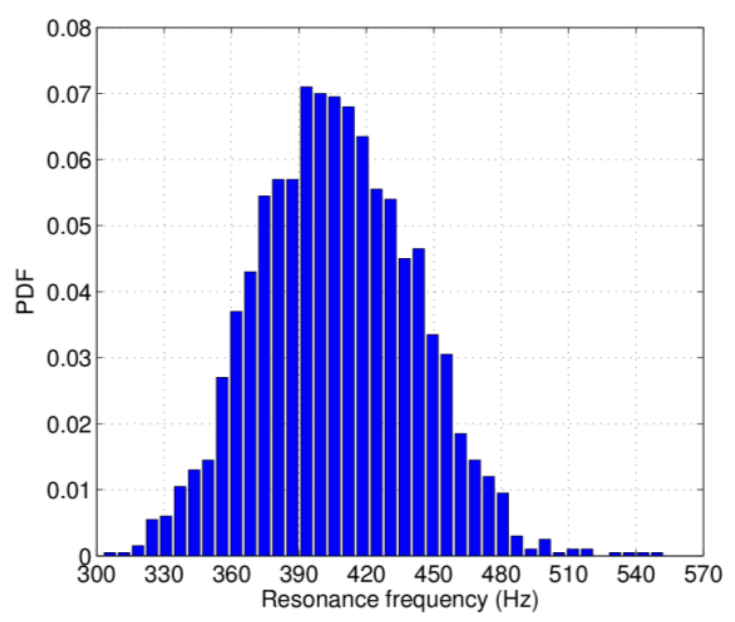

(b)

Figure 4.5 Probability density function of (a) harvested power and (b) resonance frequency.

Due to the variability of the parameters, the resonance frequencies of the samples have a large deviation from one sample to another and this can significantly decrease the performance of the harvester. Figure $4.5 \mathrm{~b}$ shows that the resonance frequency of the harvester considering mean parameters is $406 \mathrm{~Hz}$, however, there are many samples which have resonance frequencies either greater or less than the mean value. Because of this variability in resonance frequency, the harvested power of most samples deviates from the power of the system with the mean parameters when it is excited at the mean/nominal resonance frequency (see Figure 4.5a). In order to compensate for the effect of manufacturing uncertainties, the resonance frequency of samples can be adjusted by applying voltage to the electrodes. Figure 4.6a shows that by applying voltage to the straight electrodes, the resonance frequency of the micro-beam decreases due to the softening nonlinearity of the electrostatic field. Considering this nonlinearity, there are multiple solutions for the micro beam response within the frequency range close to the frequency of the vibration source. In order to harvest more power, the micro-beam response should be at the higher of the two solutions and close to the resonance frequency. However, being at the higher solution depends on the initial conditions and therefore the response at the higher amplitude cannot be guaranteed. Using applied DC voltage can ensure that the response of the harvester will be in the higher solution. For a given excitation frequency if the harvester response happens to be in the lower amplitude solution the DC voltage is increased until a region is reached where the harvester only 
has a single solution. The DC voltage is then slowly reduced, and the harvester follows the high amplitude solution until the resonance is obtained.

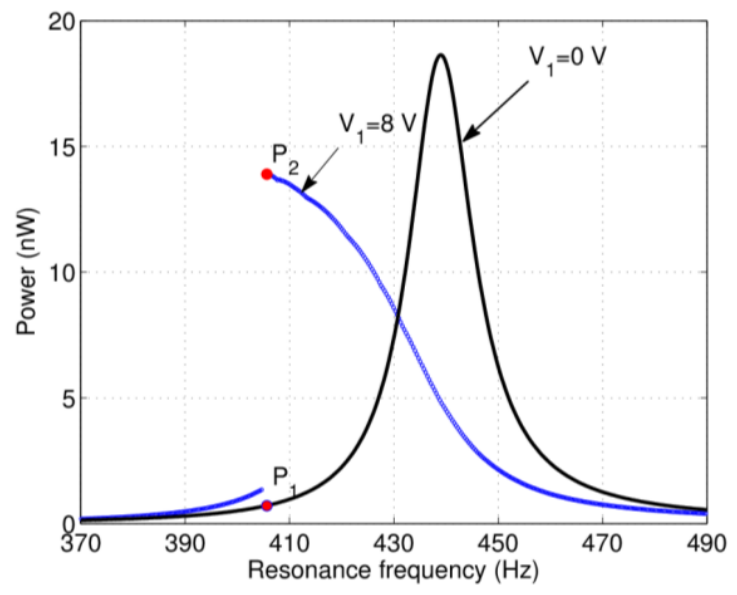

(a)

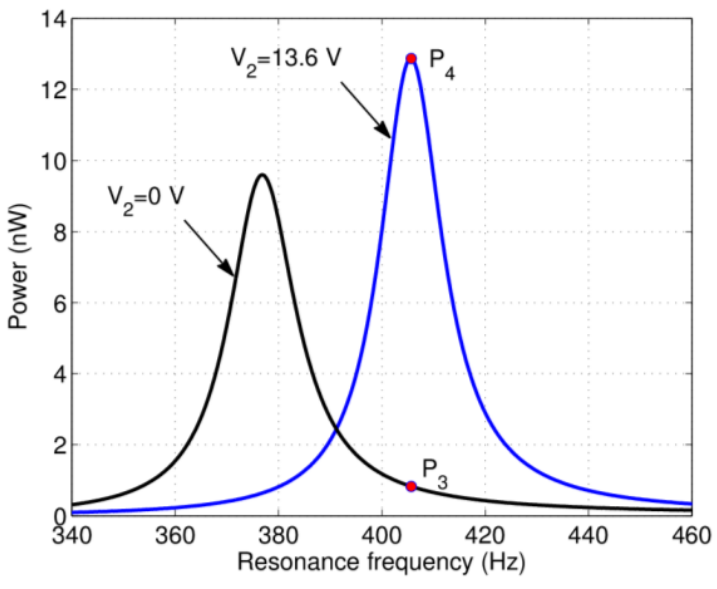

(b)

Figure 4.6 Tuning resonance frequency of microbeam using (a) softening $\left(d_{2}-d_{1}=\right.$ $0.5 \mathrm{~L})$ and $(\mathrm{b})$ hardening $\left(\alpha=30^{\circ}\right)$ mechanism

As shown in Figure 4.6a, by applying $8 \mathrm{~V}$ to the electrodes, the resonance frequency of the sample is decreased by 7.5 percent to match the vibration source frequency. Consequently, the harvested power can be increased by $13.2 \mathrm{nW}$. Figure $4.6 \mathrm{~b}$ shows that the resonance frequency of the micro-beam can be increased by applying a follower force. In Figure 4.6b, an arbitrary sample with a resonance frequency of less than $406 \mathrm{~Hz}$ has been considered. Using the hardening mechanism and applying $13.6 \mathrm{~V}$, the resonance frequency of the sample can be increased by $7.7 \%$ and therefore more power can be harvested.

In both mechanisms, the resonance frequency of the sample is tuned based on the electrostatic force. The magnitude of this force can be controlled by voltage, air gap and the overlapping area between electrodes. Generally, the air gap and overlapping area are considered to be design parameters and they are constant. However, based on Table 4.2, the air gaps between electrodes will be affected by manufacturing uncertainties. Therefore, depending on the air gaps between electrodes, the resonance frequency of the sample can be tuned by applying DC voltage. In the hardening mechanism, by applying voltage to the arc-shape electrodes, the resonance frequency of the harvester is changed linearly. However, due to the geometric configuration of the electrodes, in the softening mechanism the behavior of the harvester is affected by electrostatic nonlinearity. 


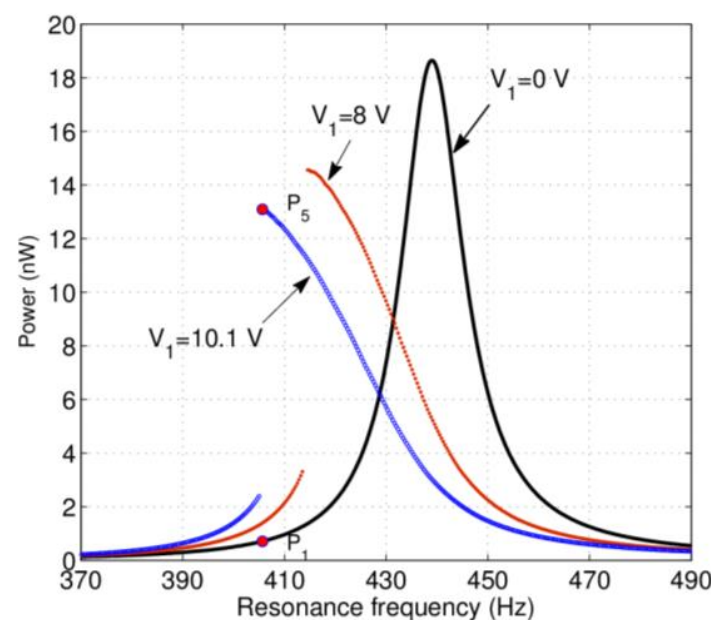

(a)

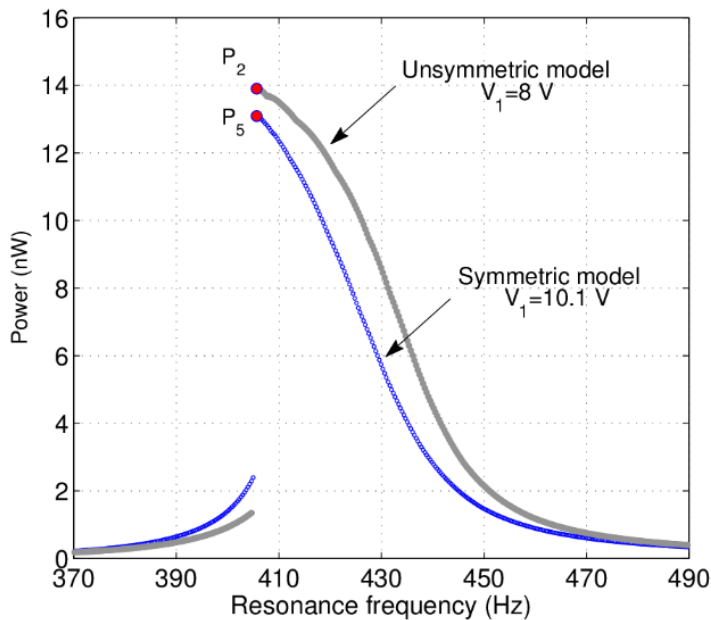

(b)

Figure 4.7 (a) Tuning resonance frequency of symmetrical model $\left(g_{01}=g_{02}=40 \mu \mathrm{m}\right)$

(b) comparison of symmetrical and unsymmetrical model $\left(g_{01}=44.6 \mu \mathrm{m}, g_{02}=\right.$

In addition, due to variabilities in the air gap between two straight electrodes, the system may become unsymmetrical. According to equation (4.5), the amplitude of the electrostatic force can be controlled by applying a DC voltage $\left(V_{1}\right)$ and changing the air gap between electrodes. As shown in Figure 4.7a, by considering equal initial gaps between electrodes $\left(g_{01}=g_{02}=40 \mu \mathrm{m}\right)$, the resonance frequency of the sample can be tuned to the nominal frequency by applying $10.1 \mathrm{~V}$ to the electrodes. However, by including the variabilities in the air gaps, the resonance frequency for the given sample may be changed by applying $8 \mathrm{~V}$ to the electrodes (See Figure $4.7 \mathrm{~b}$ ). Therefore, in comparison with the symmetrical model, depending on the initial gaps between electrodes in the unsymmetrical model, the applied DC voltage may either be increased or decreased. In addition, as shown in Figure 4.8 the output power due to the steady state response for the unsymmetrical model can be different in comparison with the symmetrical model. In the unsymmetrical model, due to the nonzero static deflection, the output voltage will be affected by a DC offset. Therefore, the output voltage will swing between two differe nt values, instead of the usual $+v_{a c}$ and $-v_{a c}$. Consequently, there will be double peaks in the steady state response of the output power in the unsymmetrical model (see figure 4.8a). 


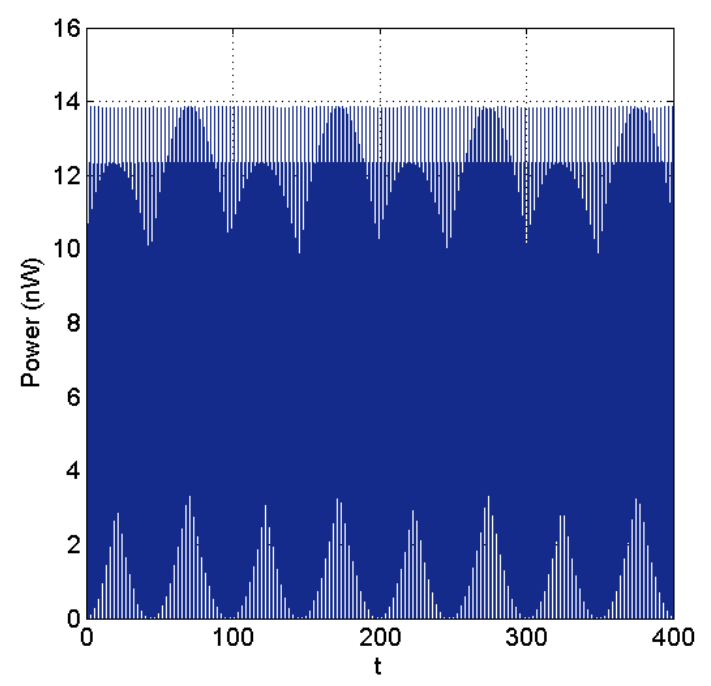

(a)

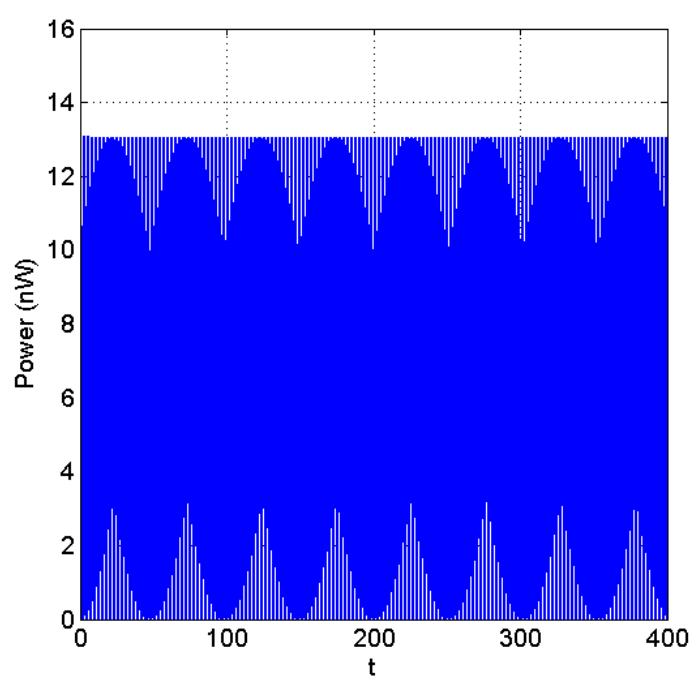

(b)

Figure 4.8 Output power due to the steady state response at $406 \mathrm{~Hz}$ (a) unsymmetrical model $\left(V_{1}=8 \mathrm{~V}\right)(\mathrm{b})$ symmetrical $\left(V_{1}=10.1 \mathrm{~V}\right)$

Generally, in nonlinear energy harvesters, maximum power can be harvested when the harvester responds on the upper branch in the vicinity of its resonance frequency. For any changes in the initial condition, the harvester tends to jump down to the lower branch, thereby decreasing harvested power significantly. As Figure 4.9a, by jumping down from point $P_{2}$ in Figure 4.6b, the harvested power decreases by $89 \%$. As shown in the time history of the power in Figure 4.9a, once the transient response is eliminated the system converges to the lower harvested power $\left(P_{0}\right)$. In order to tackle with this problem, Figure $4.9 \mathrm{~b}$ shows that in the case of jumping down to the lower solution $\left(P_{0}\right)$, the applied DC voltage is increased until a region is reached where the harvester only has a single solution. Then by delivering a gradually decreasing voltage in the fixed frequency direction, the harvester follows the high amplitude solution until resonance is obtained. The voltage source in both the symmetrical and unsymmetrical models can be charged through the harvested power from the electrostatic side. 


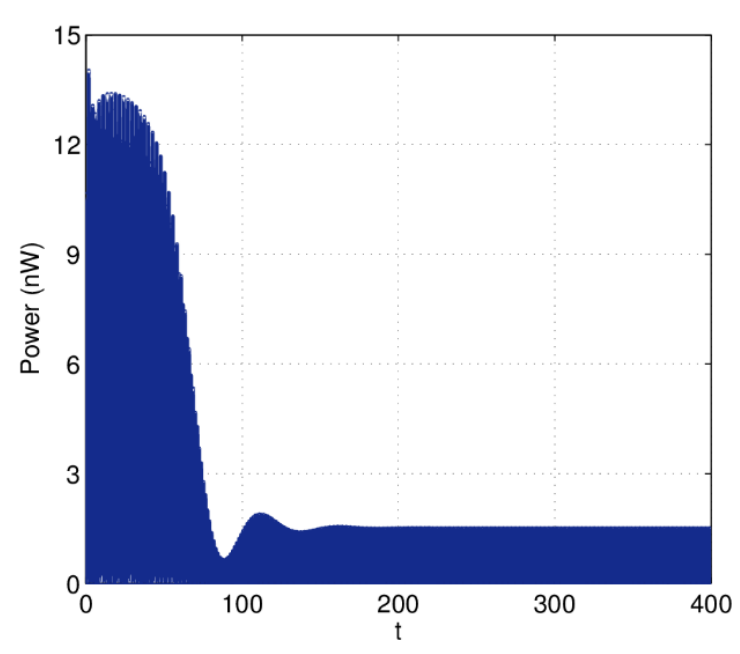

(a)

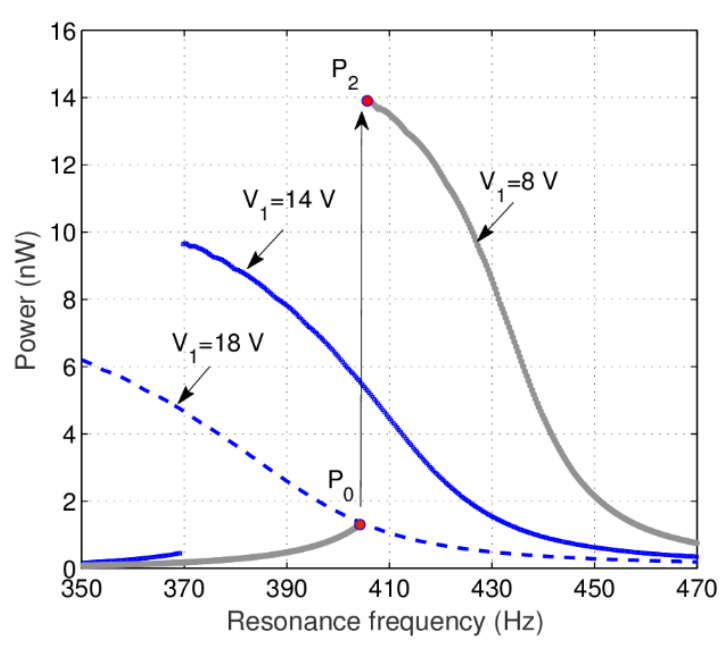

(b)

Figure 4.9 (a) Output power of the harvester on the lower branch $\left(\Omega=406 \mathrm{~Hz}, V_{1}=\right.$

$8 \mathrm{~V}$ ) (b) Moving from the lower branch to the higher by decreasing the voltage.

As discussed in Chapter 2, electrostatic harvesters require an energy cycle to convert mechanical energy to electrical energy. The energy conversion cycles mostly rely on charge or voltage constraint concepts. In both cycles, electrical charge is stored in a variable capacitor when its capacitance is high. Then the capacitance of the capacitor is reduced by mechanical vibration, and eventually the capacitor will be discharged.

Considering the voltage constraint cycle as shown in Figure 4.10a, there are two variable capacitors between the beam and the straight electrodes. In each cycle of vibration, these capacitors are charged and discharged continuously, and they can charge the voltage source $\left(V_{1}\right)$ based on the voltage constraint cycle. Therefore, in both symmetrical and unsymmetrical models, the voltage source is self-chargeable and the harvested power from the electrostatic side is used to keep the voltage source constant.

Considering all samples, by applying different voltages to the electrodes, the resonance frequency of the samples matches the excitation frequency and more power can be harvested, as shown in Figure 4.11. Figure 4.12 shows that by applying DC voltage to the straight electrodes $\left(V_{1}\right)$ up to $26.6 \mathrm{~V}$ and the arch-electrodes $\left(V_{2}\right)$ up to $24 \mathrm{~V}$, the harvested power of the samples can be improved significantly. 


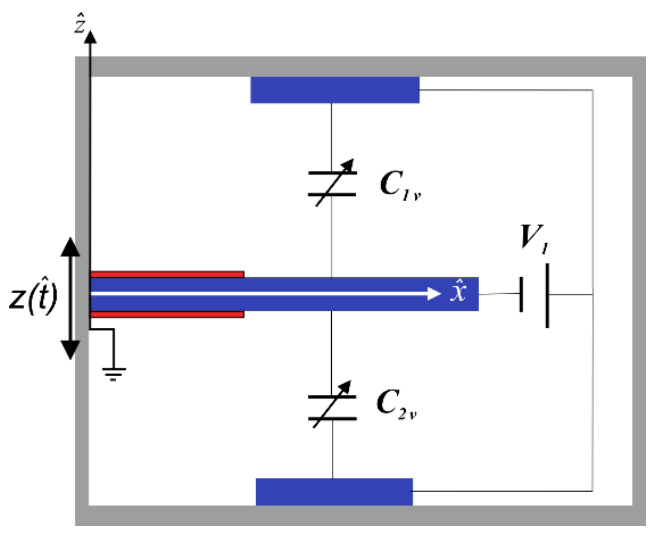

(a)

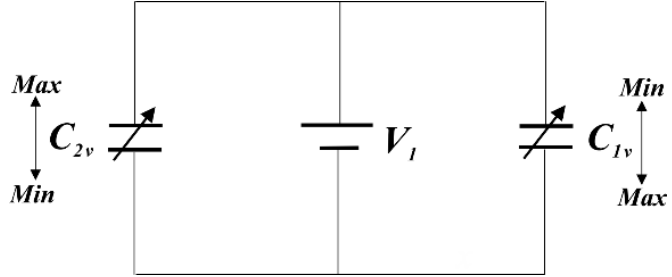

(b)

Figure 4.10 (a)Variable capacitors in the proposed model (b) Electrical circuit

Consequently, in comparison with Figure 4.5a, most samples are shifted to the region around the power of the system with the mean parameters. As shown in Figure 4.12a, the mean DC voltage applied to the straight electrodes (point A) is $8.5 \mathrm{~V}$, and in most cases the harvested power of the samples is close to the power of the system with the mean parameters.

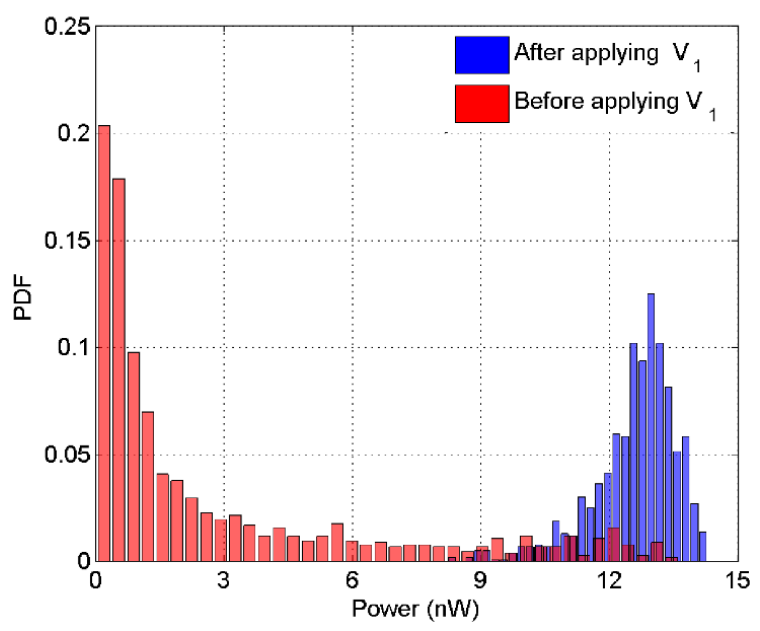

(a)

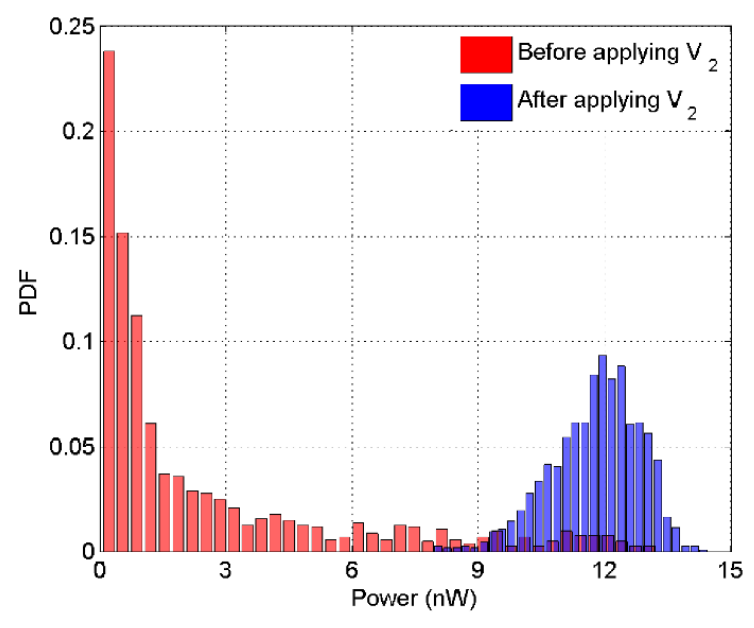

(b)

Figure 4.11 Harvested power of samples based on (a) softening and (b) hardening mechanism

However, for the hardening mechanism, the mean applied DC voltage (point B in figure $4.12 \mathrm{~b}$ ) is $12.2 \mathrm{~V}$, which is $3.7 \mathrm{~V}$ greater than the mean applied DC voltage for the softening mechanism. Furthermore, the mean harvested power of the samples for the hardening mechanism is less than $12 \mathrm{nW}$. Therefore, the electrostatic nonlinearity in the 
softening mechanism can make the tuning mechanism more efficient in comparison with the hardening mechanism.

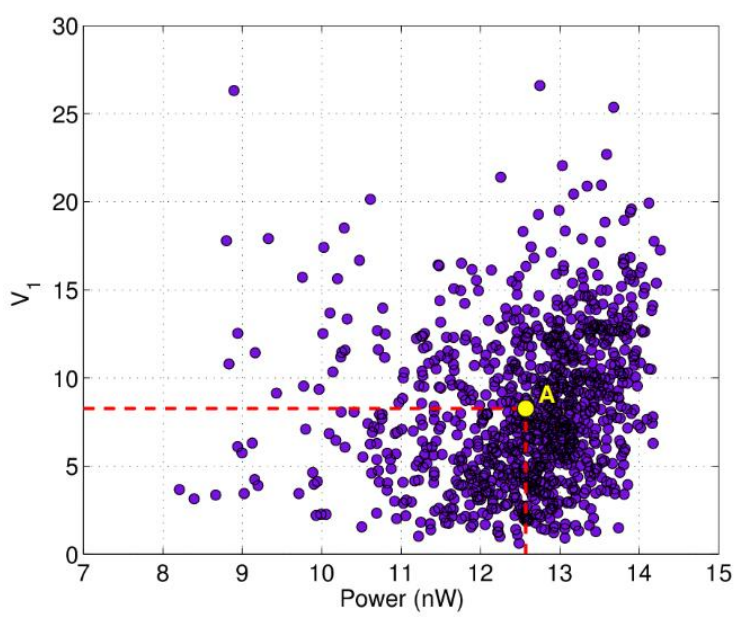

(a)

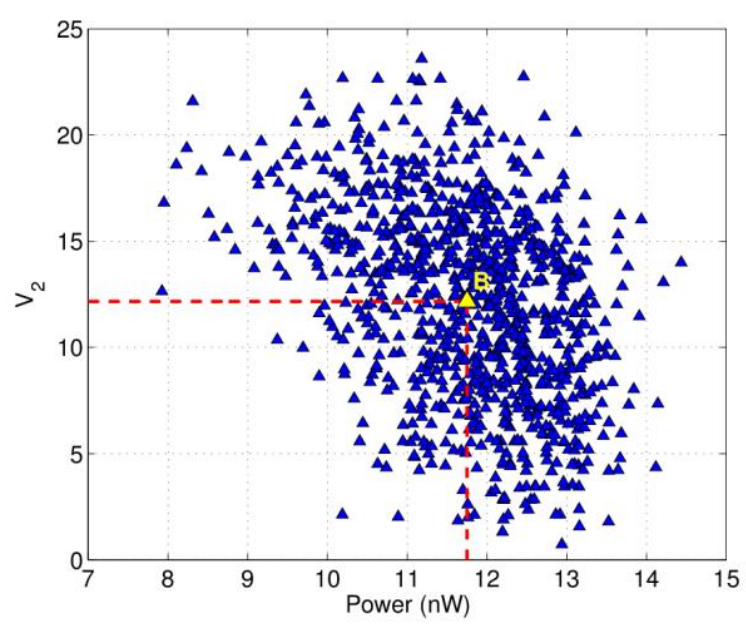

(b)

Figure 4.12 (a) Applied DC voltage versus harvested power for softening mechanism and (b) hardening mechanism

In the current analysis, a constant optimal resistance $(70 \mathrm{k} \Omega)$ is used for all samples, however this resistance can be optimized for each sample [108]. In addition, since the axial deflection of the beam is negligible, the power loss of voltage source $V_{2}$ is small. Considering the results of both mechanisms, as shown in Figure 4.13 by using the electrostatic force in both mechanisms, the effect of manufacturing uncertainties can be compensated for and after tuning the resonance frequencies of the samples, the harvested power of the samples varies between 8 to $14 \mathrm{nW}$. 


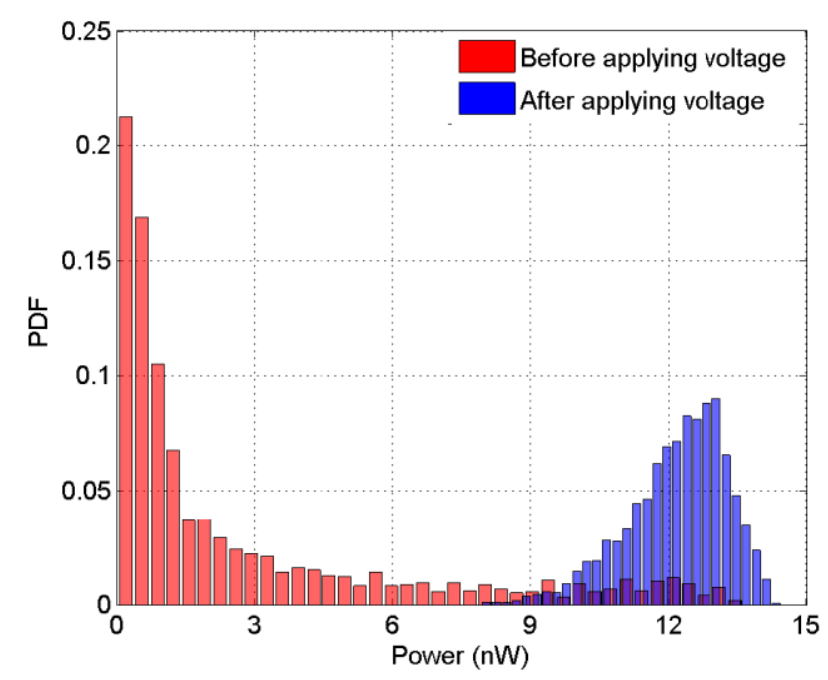

Figure 4.13 Harvested power of samples before and after applying DC voltage

\subsection{Closure}

In this chapter, the effect of manufacturing uncertainties on the performance of MEMS piezoelectric harvesters has been investigated. The steady state solution was obtained by using the shooting method and 2,000 samples were considered based on Monte Carlo Simulation. From this study, the following important conclusions were drawn:

The results showed that variability in a MEMS harvester will significantly reduce its performance. This is because the resonance frequencies of the samples in most cases were far away from the excitation frequency and resulted in lower harvested power. It should be noted that the experimental data in the literature was used to randomise the model parameters.

We propose two tuning mechanisms that can be used to compensate for the effect of manufacturing uncertainty. For each sample, depending on its resonance frequency, appropriate DC voltage was applied. Based on these mechanisms, it was observed that the harvested power can be increased by applying DC voltage to the straight electrodes and arc-shaped electrode up to $26.6 \mathrm{~V}$ and $24 \mathrm{~V}$, respectively. 
* The problem that we encountered in this study was a nonlinear and uncertain dynamic problem. This is a very challenging problem and there is little attention to it in the literature. I found that the use of previous semi-analytical solutions described by authors in previous studies is not feasible. The use of time-integration is also extremely time-consuming in this problem (considering that we are only interested in the steady state solution). Therefore, (for the first time to my knowledge) we proposed the combined version of a shooting method and Monte Carlo Simulation which was found to be efficient enough to solve this uncertain nonlinear problem. 


\section{Chapter 5: Experimental studies of an energy harvester with adjustable resonance frequency in macroscale}

This chapter presents an experimental setup to tune the resonance frequency of a bimorph piezoelectric energy harvester. The aim of this chapter is to simulate electrostatic force via electromagnets to illustrate the concept of broadband energy harvesting in macro scales. To this end, firstly the dynamic behaviour of the model based on the electromagnetic forces is investigated. Following this, piezoelectric patches are bonded to the beam to show the application of the proposed model in energy harvesting.

\subsection{Experimental set up}

As shown in Figure 5.1, the model is a cantilever beam with two tip permanent magnets (N42 Neodymium magnets) in both sides of the beam. In addition, there is an electromagnet in one side of the beam which can create attractive or repulsive force depending on the arrangement of the magnet poles. The geometric and other properties of the beam and tip magnets have been listed in Table 5.1. The tip magnets and electromagnet pull strength are $2 \mathrm{~kg}$ and $25 \mathrm{~kg}$ respectively. Generally, the pull strength is the highest possible holding power of the magnet, measured in kilograms. It is noticeable that the equations and the solutions considered earlier for a microscale model can be used for a macroscale model. 
Table 5.1 Geometrical and material properties of the beam and tip magnets

\begin{tabular}{lc}
\hline Design Variable & Value \\
\hline Length of the beam, $L(\mathrm{~mm})$ & 312 \\
Width of the beam, $b(\mathrm{~mm})$ & 30 \\
Thickness of the beam, $h_{\tilde{s}}(\mathrm{~mm})$ & 1.1 \\
Thickness of the tip magnets, $h_{0}(\mathrm{~mm})$ & 1.5 \\
Diameter of tip magnets, $D_{0}(\mathrm{~mm})$ & 20 \\
Young's modulus of the beam, $E_{\tilde{s}}(\mathrm{GPa})$ & 205.5 \\
Young's modulus of permanent magnets, $E_{0}(\mathrm{GPa})$ & 160 \\
Density of beam, $\rho_{\tilde{s}}\left(\mathrm{~kg} / \mathrm{m}^{3}\right)$ & 8040 \\
Density of permanent magnets, $\rho_{0}\left(\mathrm{~kg} / \mathrm{m}^{3}\right)$ & 7500 \\
Viscous damping, $c_{a}(\mathrm{~N} . \mathrm{s} / \mathrm{m})$ & 0.455
\end{tabular}

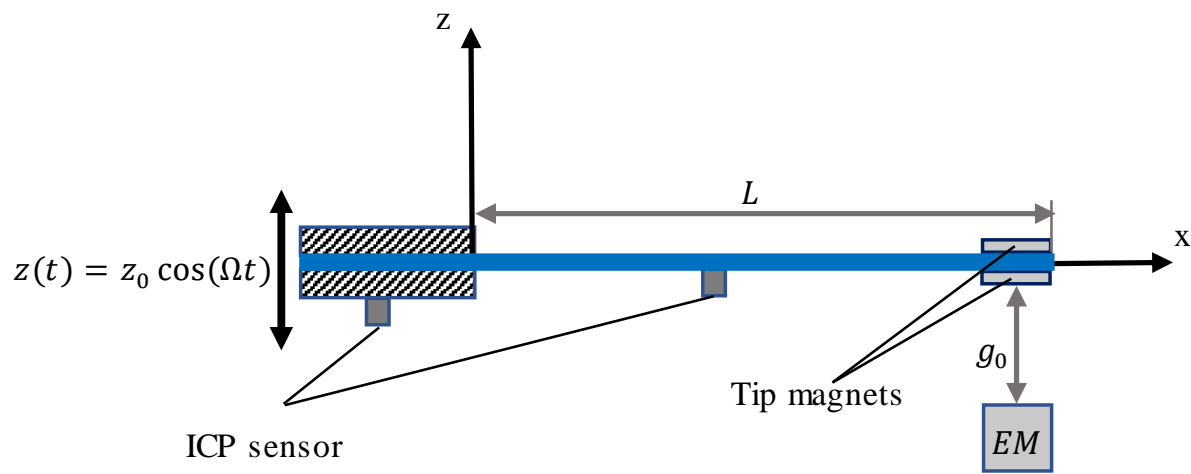

Figure 5.1 Schematic of the proposed model (Top view)

\subsection{Characterization of electromagnetic force}

In this section, the electromagnetic force between magnets is investigated experimentally. Generally, the electromagnetic force between the beam and the electromagnet depends on the air gap and the DC voltage applied to the electromagnet. Figure 5.2 shows the effect of the air gap on the electromagnetic force between the beam and the electromagnet. In this case, there is no DC voltage applied to the electromagnet, and the tip deflection of the beam is measured by a laser displacement sensor. Decreasing the air gap between the beam and the electromagnet, the beam is attracted to the electromagnet. As shown in Figure 5.2 , by varying the air gap from $50 \mathrm{~mm}$ to $35 \mathrm{~mm}$, the tip deflection of the beam increases linearly. However, in air gaps of less than $35 \mathrm{~mm}$, the system becomes nonlinear and suddenly, at $18 \mathrm{~mm}$, the beam becomes stuck to the electromagnet. Therefore, 
depending on the distance between magnets, there can be some initial deflection on the beam and it is more obvious when the distance is less than $40 \mathrm{~mm}$.

Alternatively, by considering the attractive force between the beam and electromagnet, the deflection of the beam can be increased by increasing the DC voltage applied to the electromagnet. Furthermore, depending on the distance between magnets, the deflection of the beam can be increased either linearly or nonlinearly.

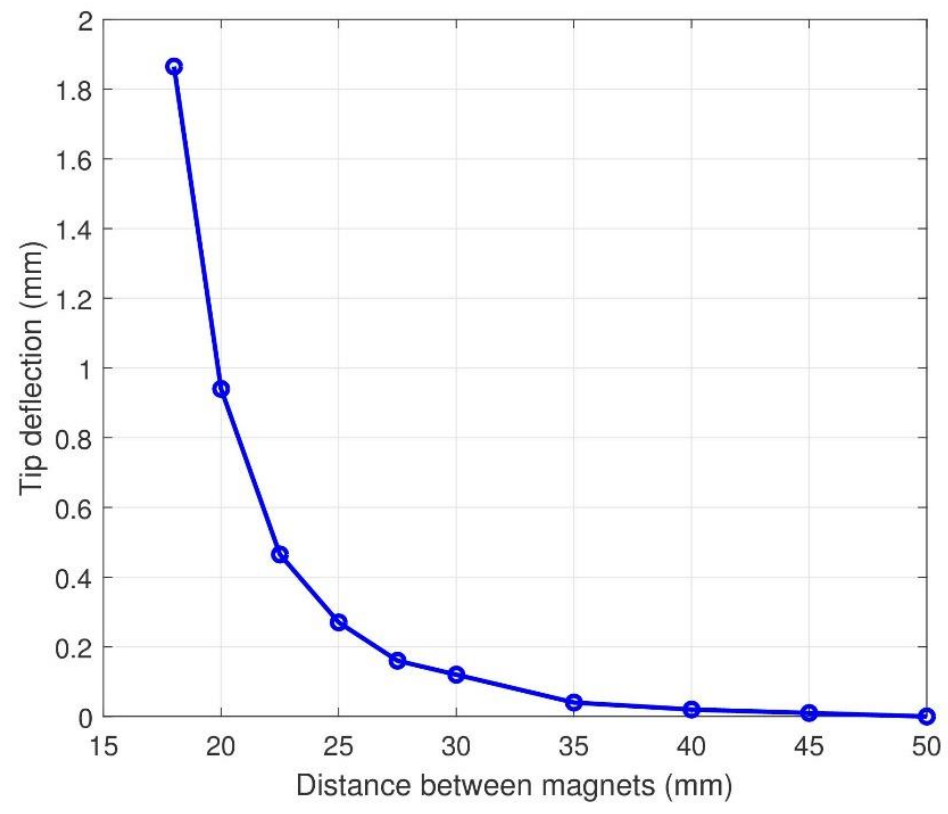

Figure 5.2 Tip deflection of the beam versus distance between magnets $\left(V_{D C}=0 \mathrm{~V}\right)$

Based on the result shown in Figure 5.3, the nonlinearity increases when the distance between magnets is less than $30 \mathrm{~mm}$. Note that in the case of a $20 \mathrm{~mm}$ air gap between magnets, the beam becomes stuck to the electromagnet when applying voltages of more than $6 \mathrm{~V}$ to the electromagnet. 


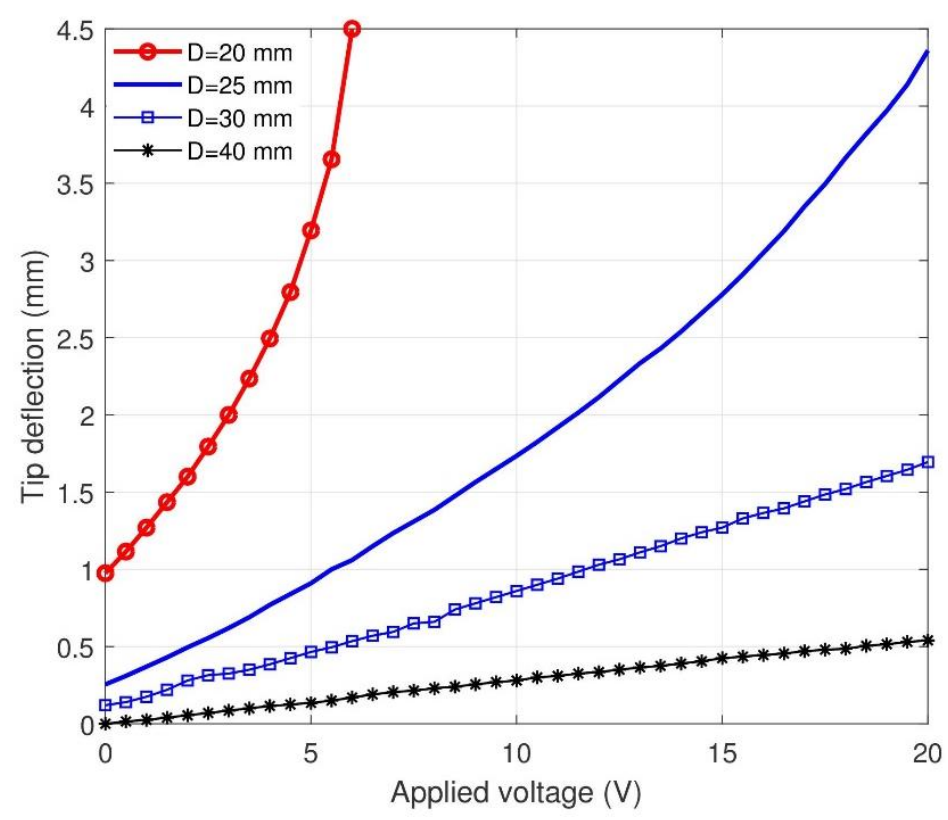

Figure 5.3 Tip deflection of the beam versus DC voltage applied to the electromagnet

As previously noted, the electromagnetic force between magnets can be either attractive or repulsive. In Figure 5.4, these two possible scenarios and their effect on the beam at a $25 \mathrm{~mm}$ air gap between magnets are shown. As illustrated in Figure 5.4, the attractive force is more powerful than the repulsive force. For instance, considering the attractive force at $20 \mathrm{~V}$ applied voltage to the electromagnet, the deflection of the beam is 2.5 times greater than the same scenario when applying the repulsive force. The main reason that the repulsive force is weaker than the attractive one is related to this fact that the permanent magnet is always attracting the iron core inside the electromagnet. By using air-core electromagnets, the repulsive force between the electromagnet and the permanent magnet can be same as the attractive one. In addition, when using the repulsive force scenario, the deflection of the beam increases almost linearly by increasing the DC voltage applied. 


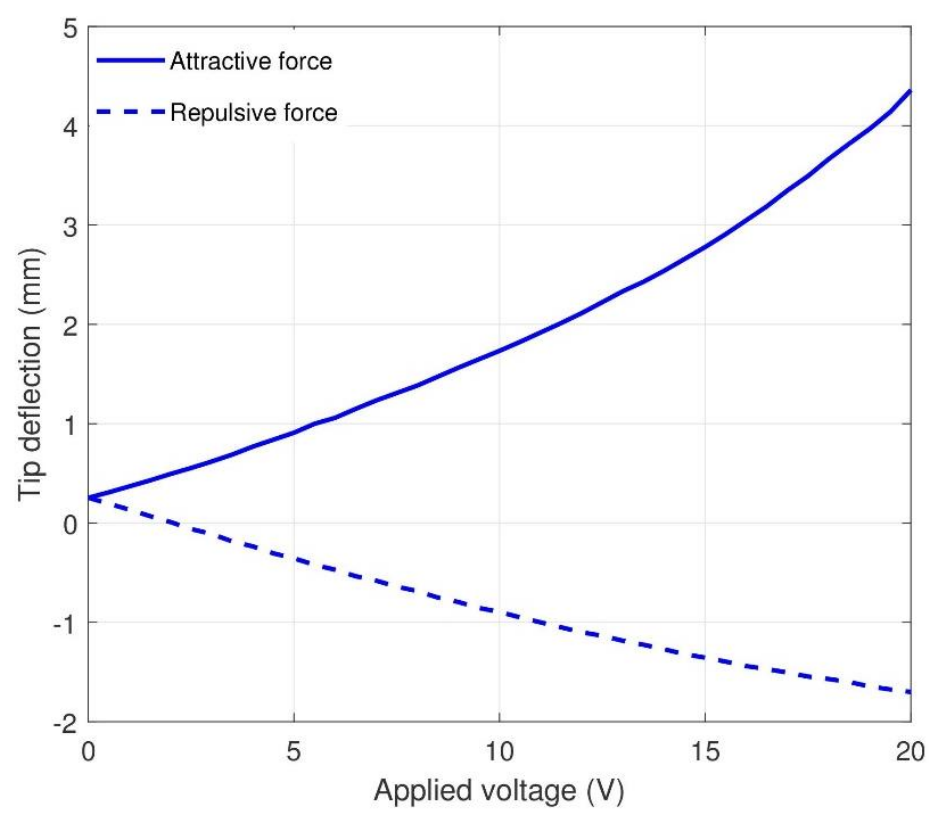

Figure 5.4 Tip deflection of the beam versus DC voltage applied to the electromagnet at $\mathrm{D}=25 \mathrm{~mm}$

\subsection{Frequency analysis}

The aim of this section to study the dynamics of the proposed model. To carry out the dynamic test, we have used an APS Dynamics Model 420-HF electrodynamic shaker (APS Dynamic Juan Capistrano, CA) as an excitation source, powered by an APS amplifier Model 145. As shown in Figure 5.5, two ICP sensors have been used to measure the acceleration of the beam and the base. The analogue data measured by these two sensors is converted into digital data using Abacus dynamic signal analysis hardware and is then sent to a SignalCalc analyser.

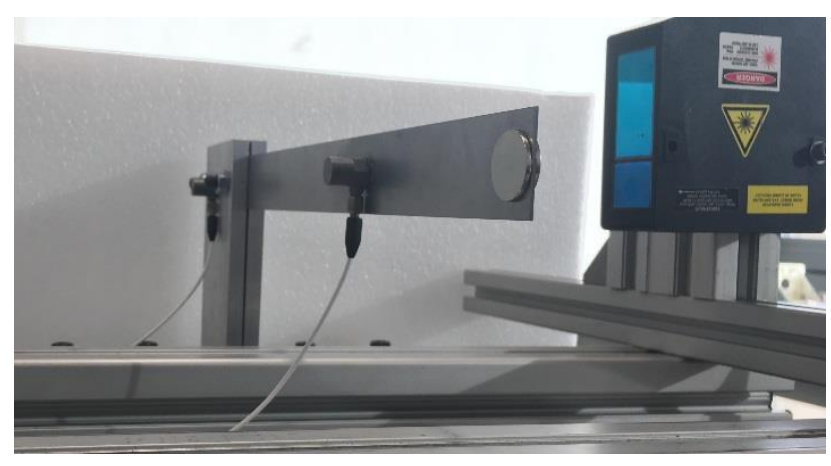

Figure 5.5 linear model 
To identify the first two resonance frequencies of the beam, here we have used a random burst excitation. As Figure 5.6 shows, the first and second resonance frequencies of the beam are $7.7 \mathrm{~Hz}$ and $50 \mathrm{~Hz}$, respectively. Using random burst excitation is a straightforward and fast way to determine the resonance frequencies of the beam. It is noticeable that random burst excitation method takes the average of several FRFs and its accuracy can be increased by increasing the number of taken FRFs. In the case of there being nonlinearity in the system, we must use sin-step analys is to capture the nonlinear behaviour of the system properly.

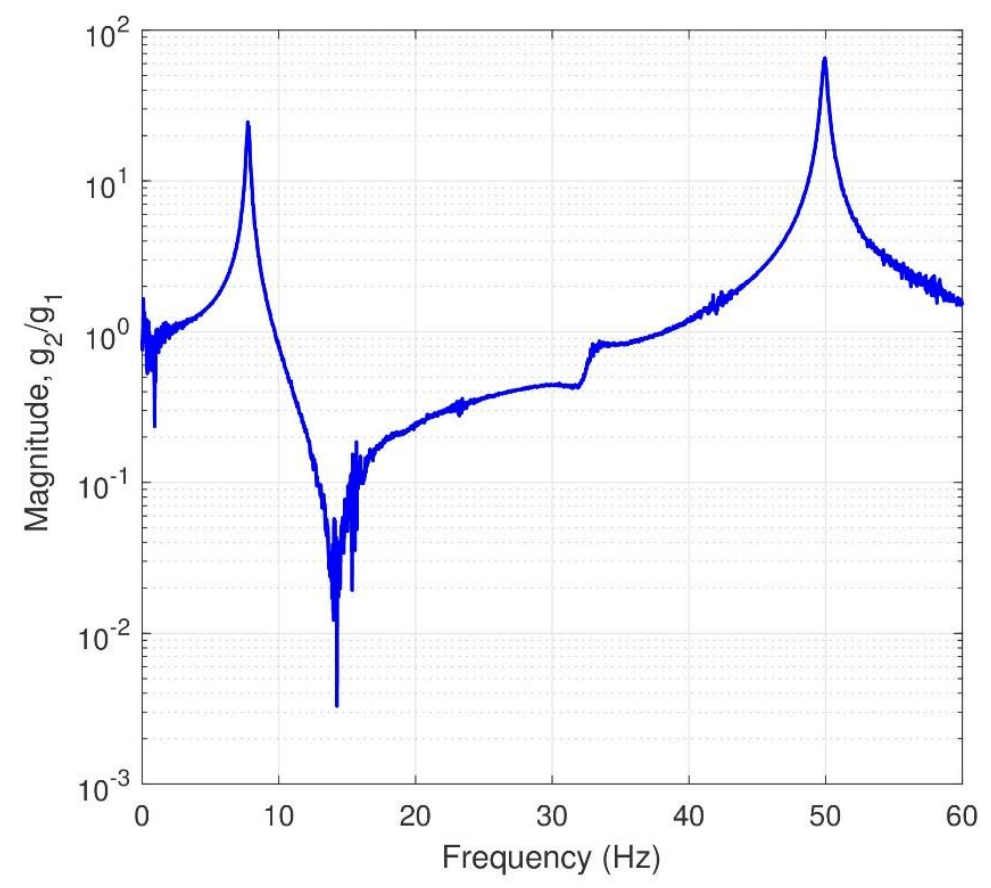

Figure 5.6 FRF of the linear model

As stated earlier, the aim of this chapter is to simulate electrostatic force via using electromagnets to illustrate the concept of broadband energy harvesting in macro scales. To this end, we used two identical electromagnets in both sides of the beam (See Figure 5.7). Depending on the arrangement of the magnet poles, the electromagnetic force between magnets can be either attractive or repulsive. 


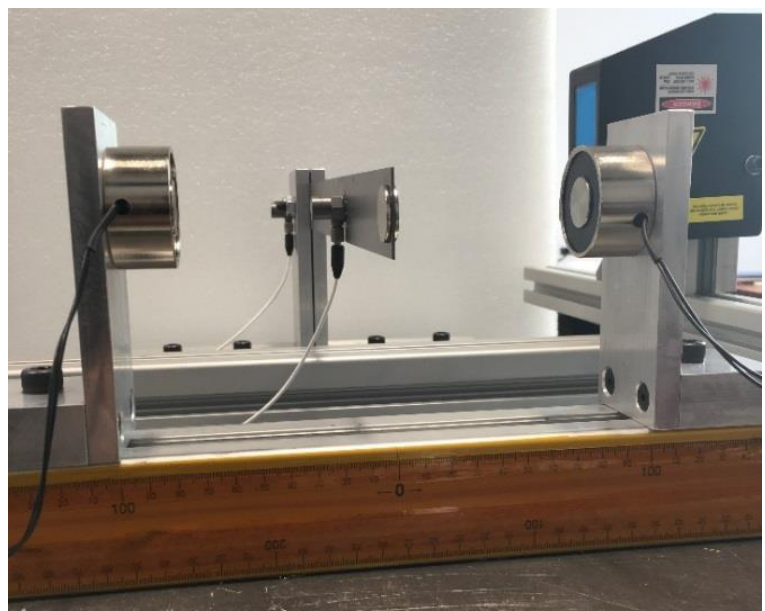

Figure 5.7 Using electromagnetic device to control the resonance frequency of the beam

Firstly, we consider the attractive force between magnets. As shown in Figure 5.2, there is a displacement dependent electromagnetic force between magnets when there is no DC voltage applied to the electromagnet. Figure 5.8 investigates the effect of the magnetic force on the dynamics of the system for different air gaps between magnets $\left(g_{0}\right)$.

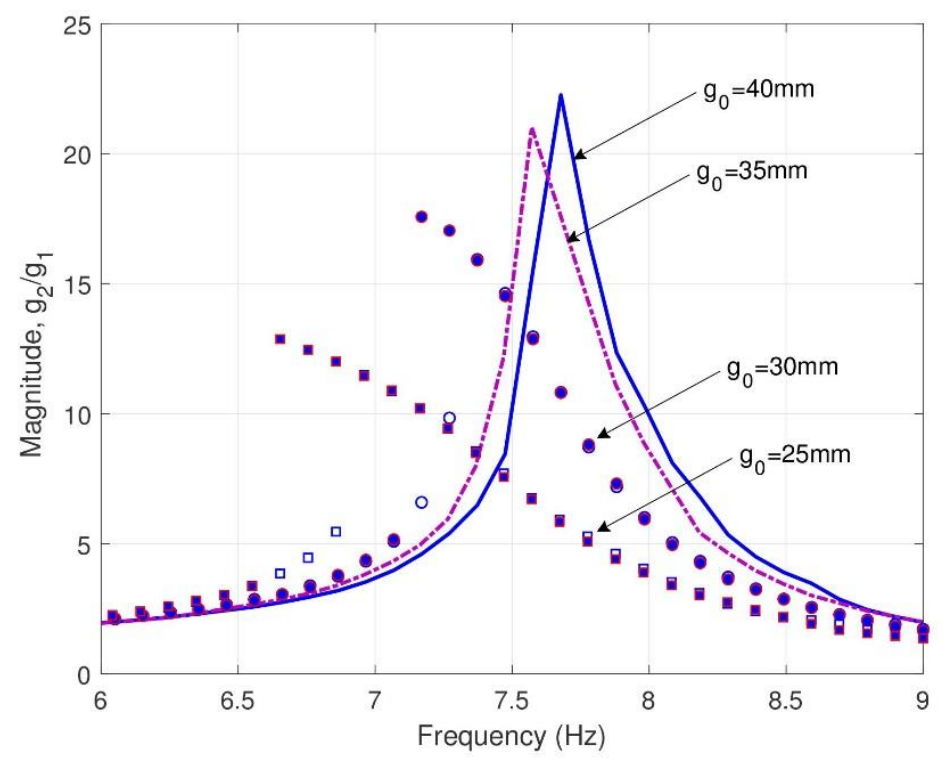

Figure 5.8 FRFs for different air gaps between magnets $\left(g_{0}\right)$

As Figure 5.8 shows, for $g_{0}=40 \mathrm{~mm}$, the electromagnets have no significant effect on the beam and the resonance frequency remains the same as the initial linear system (See Figure 5.6). However, by decreasing $g_{0}$ to $35 \mathrm{~mm}$ the resonance frequency of the beam 
decreases linearly. By further reducing the air gap between magnets, the system becomes nonlinear and the frequency response of the beam is affected by the softening nonlinearity. This behaviour is similar to the electrostatic model, discussed in previous chapters. By applying voltage to the electromagnet, the attractive force between the magnets increases.

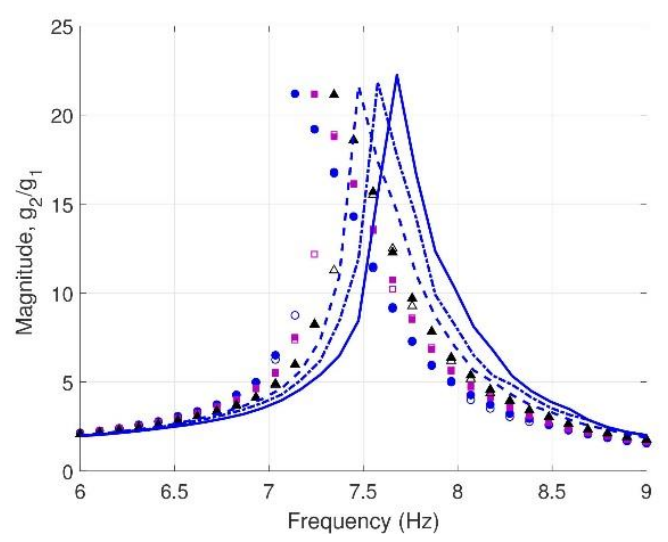

(a) $g_{0}=40 \mathrm{~mm}$

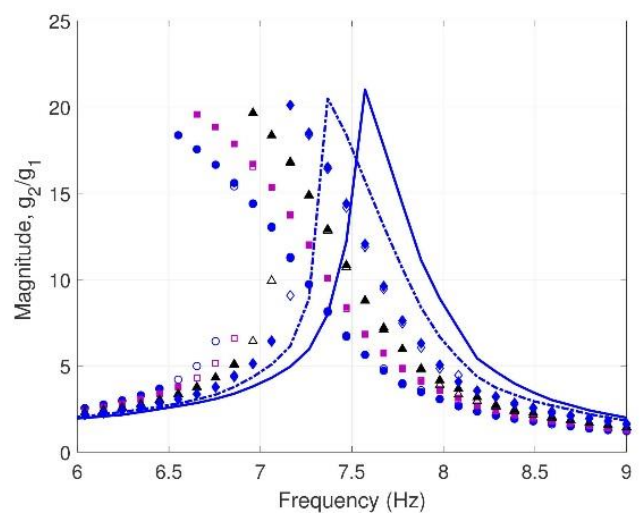

(c) $g_{0}=35 \mathrm{~mm}$

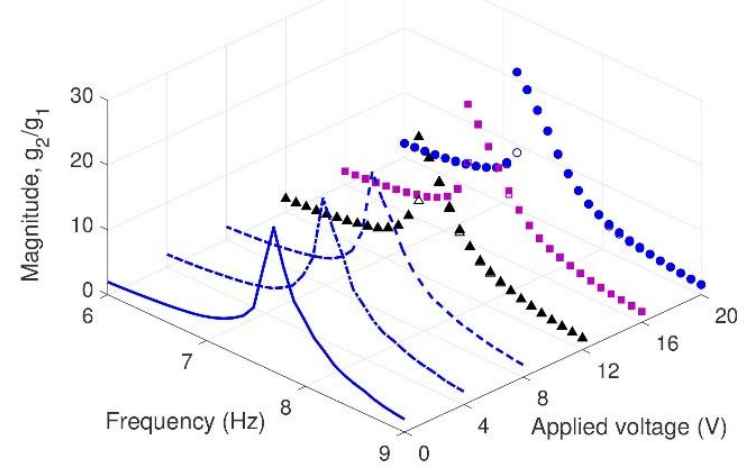

(b) $g_{0}=40 \mathrm{~mm}$

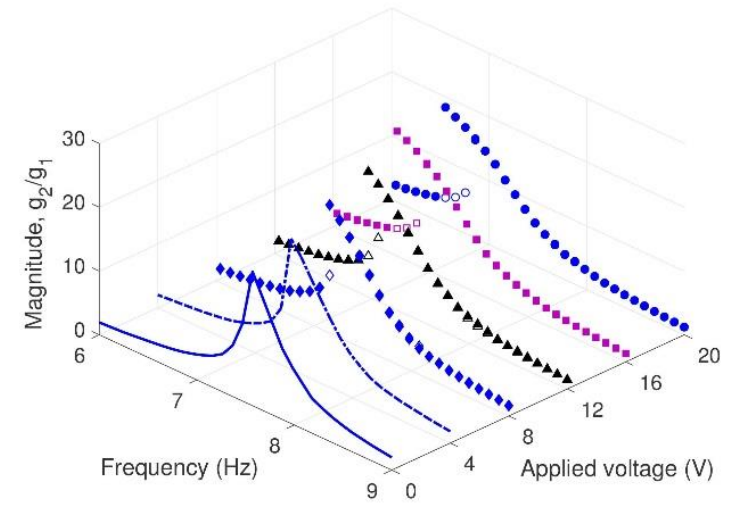

(d) $g_{0}=35 \mathrm{~mm}$

Figure 5.9 FRFs of the beam for different air gaps between magnets $\left(g_{0}\right)$

Depending on the air gap between the magnets, the resonance frequency of the beam can be tuned to lower frequencies by using applied DC voltage as a control parameter. Figure 5.9 shows the changes in the resonance frequency of the beam by applying voltage to the electromagnets for $40 \mathrm{~mm}$ and $35 \mathrm{~mm}$ air gaps between magnets. As shown in Figures 5.9a and $5.9 \mathrm{~b}$, by increasing the voltage up to $20 \mathrm{~V}$ for $g_{0}=40 \mathrm{~mm}$, the resonance frequency of the beam can be changed from 7.7 to $7.13 \mathrm{~Hz}$. By decreasing the air gap to $35 \mathrm{~mm}$, the 
resonance frequency of the beam can be tuned in the range of 7.6 to $6.5 \mathrm{~Hz}$ (see Figures $5.9 \mathrm{c}$ and $5.9 \mathrm{~d})$. In these two cases, with lower voltages applied to the electromagnets, the resonance frequency of the beam decreases linearly and when increasing the voltages applied, the behaviour of the system is affected by softening nonlinearity.

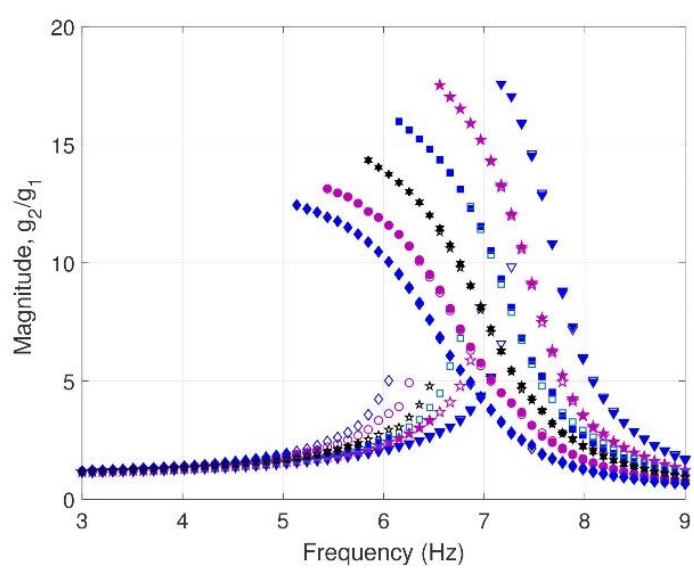

(a) $g_{0}=30 \mathrm{~mm}$

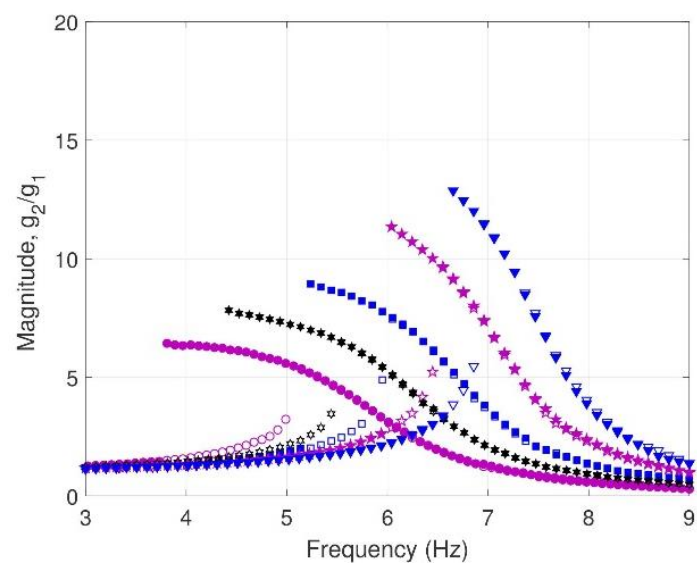

(c) $g_{0}=25 \mathrm{~mm}$

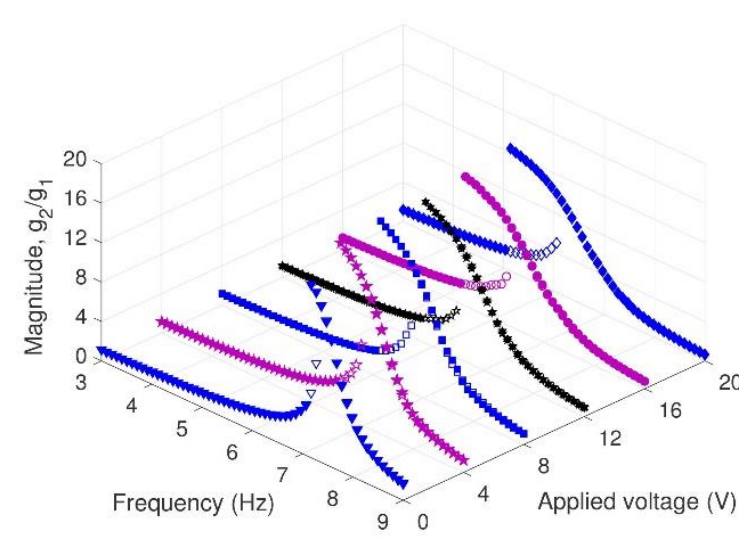

(b) $g_{0}=30 \mathrm{~mm}$

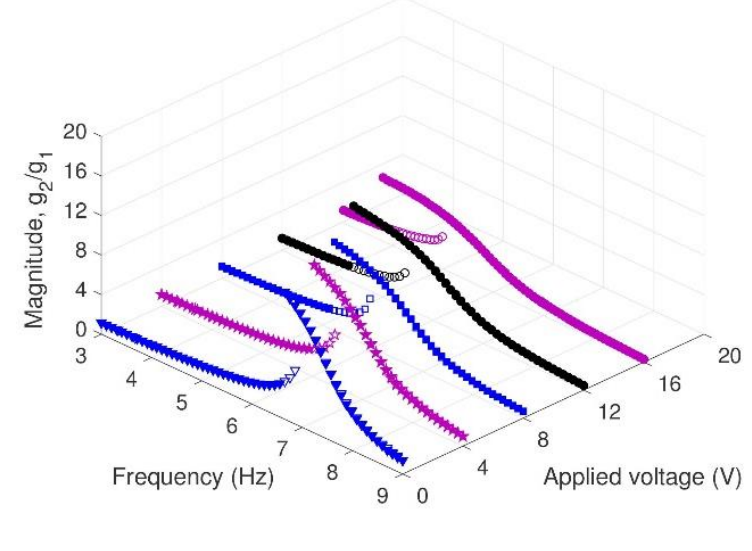

(d) $g_{0}=25 \mathrm{~mm}$

Figure 5.10 FRFs of the beam for different air gaps between magnets $\left(g_{0}\right)$

By further reducing the air gap between magnets, the range of tuning can be increased. On the other hand, the amplitude of the beam decreases. As illustrated in Figure 5.10a, at a 30mm air gap between magnets, increasing the voltage applied to the electromagnets from 0 up to $20 \mathrm{~V}$ can change the resonance frequency of the beam from 7.2 to $5.2 \mathrm{~Hz}$. However, a $5 \mathrm{~mm}$ reduction of $g_{0}$ changes the resonance frequency of the beam from 6.7 to $3.8 \mathrm{~Hz}$ (see Figure 5.10c). In addition, decreasing the air gap between magnets decreases the dynamic pull-in voltage. Considering the same level of base excitation, the 
dynamic pull-in voltage in all cases is greater than $20 \mathrm{~V}$, except in the last case $\left(g_{0}=\right.$ $25 \mathrm{~mm}$ ), where it is $16 \mathrm{~V}$.

As discussed earlier, by changing the pole arrangement of the electromagnet poles, the attractive force between the magnets can be changed to repulsive force. Figure 5.11 investigates the effect of the repulsive force on the resonance frequency of the beam when the air gap between magnets is $25 \mathrm{~mm}$.

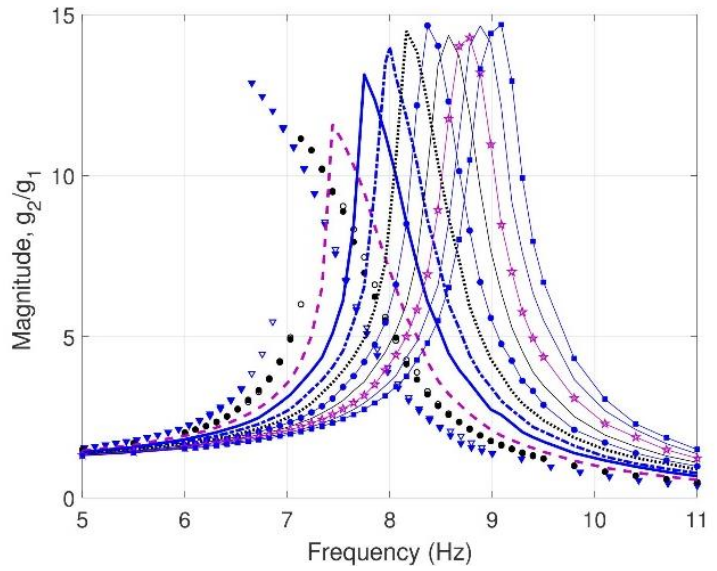

(a) $g_{0}=25 \mathrm{~mm}$

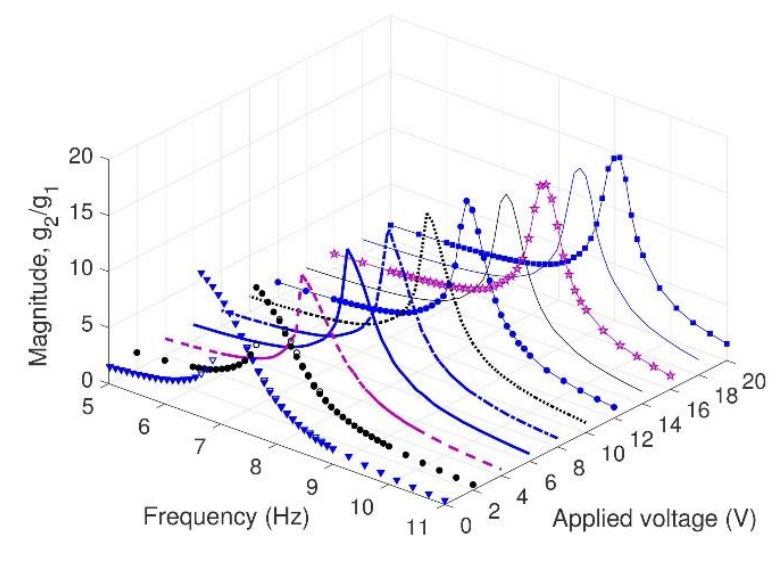

(b) $g_{0}=25 \mathrm{~mm}$

Figure 5.11 FRF of the beam for different air gaps between magnets $\left(g_{0}\right)$

As Figure 5.11 shows, by increasing the voltage applied to the electromagnets, the resonance frequency of the beam increases. Using repulsive force configuration and applying voltage up to $20 \mathrm{~V}$ increases the resonance frequency of the beam from 6.6 to 9.1 Hz. By using repulsive force between magnets, the resonance frequency of the beam can be tuned from 6.6 to $9.1 \mathrm{~Hz}$. It is noticeable that based on Figure 5.4, the repulsive force is less nonlinear in comparison with the attractive force. In addition, in repulsive force configurations, there is still a small amount of attractive force between the permanent magnets and the exterior part of the electromagnets. This attractive force is cancelled out by repulsive force when applying $2 \mathrm{~V}$ to the electromagnets in static analysis (See Figure 5.4). However, in the dynamic tests the frequency response of the beam is still affected by softening nonlinearity at $2 \mathrm{~V}$ and by increasing the voltage applied to the electromagnets, the system becomes linear due to the effect of repulsive force. Considering both repulsive and attractive force at a $25 \mathrm{~mm}$ air gap between magnets, 
the resonance frequency of the beam can be tuned from 3.8 to $9.1 \mathrm{~Hz}$. This behaviour shows the great potential of the proposed model for energy harvesting applications.

\subsection{Mathematical modelling}

This section focuses on developing a mathematical model to verify the experimental results provided in the previous section. Figure 5.12 shows the schematic of the beam from top view.

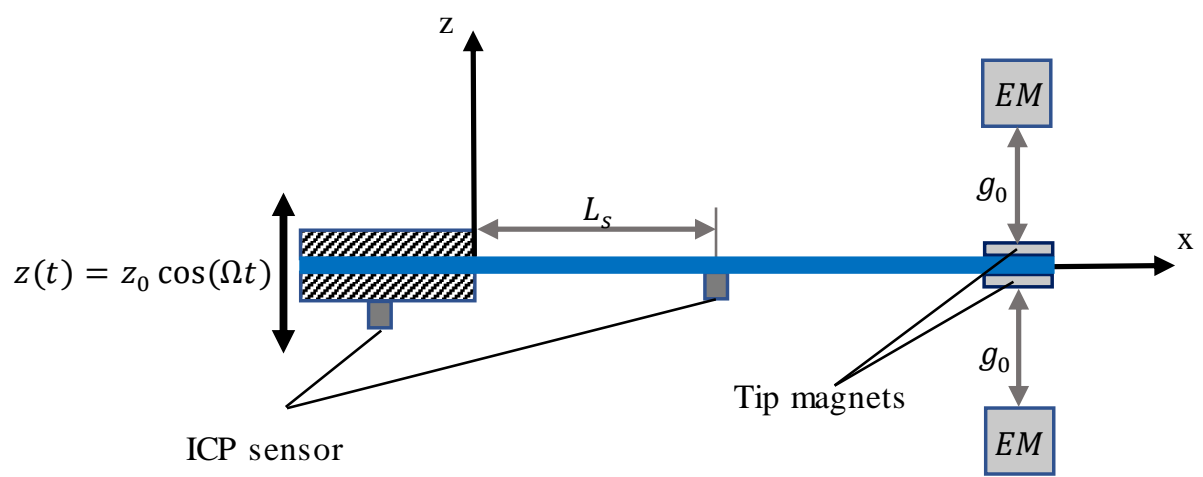

Figure 5.12 Top view of the model

As shown in Figure 5.12, two permanent magnets are attached to the tip of the beam and there are two electromagnets $(\mathrm{EM})$ which are connected to the same DC voltage. In addition, there are two ICP sensors to measure the acceleration of the beam and the base. The governing equation of transverse motion can be written as

$$
\begin{array}{r}
E I \frac{\partial^{4} w_{r e l}(x, t)}{\partial x^{4}}+\left(m+M_{s} \delta\left(x-L_{s}\right)\right) \frac{\partial^{2} w_{r e l}(x, t)}{\partial t^{2}}+c_{a} \frac{\partial w_{r e l}(x, t)}{\partial t} \\
=-\left(m+M_{s} \delta\left(x-L_{s}\right)+M_{t} \delta(x-L)\right) \frac{\partial^{2} z(t)}{\partial t^{2}}+F_{M} \delta(x-L)
\end{array}
$$

and subjected to the following boundary conditions

$$
\begin{gathered}
w_{r e l}(0, t)=0, \quad \frac{\partial w_{r e l}(0, t)}{\partial x}=0, \quad \frac{\partial^{2} w_{r e l}(L, t)}{\partial x^{2}}=0 \\
E I \frac{\partial^{3} w_{r e l}(L, t)}{\partial x^{3}}=M_{t} \frac{\partial^{2} w_{r e l}(L, t)}{\partial t^{2}}
\end{gathered}
$$


In equation (5.1), $w_{\text {rel }}(x, t)$ is the transverse deflection of the beam relative to its base at position $x$ and time $t, E$ is the Young's modulus of the beam, $c_{a}$ is the viscous air damping coefficient, $\delta(x)$ is the Dirac delta function, $F_{M}$ is the magnetic force, $z(t)$ is the base excitation function, $m$ is the mass per unit length of the beam, $M_{t}$ is the tip mass, and $M_{S}$ is the mass of the ICP sensor attached to the beam. Generally, electromagnetic force is a function of applied DC voltage to the electromagnets and the initial gap between magnets. In addition, it depends on the geometrical characteristics of the electromagnets. Having permanent magnets as a tip mass creates a magnetic force, which depends on the distance between the beam and the electromagnet.

There have been several approaches to model the magnetic force between magnets [109, 110], however, for the current configuration there is no specific equation. Therefore, here we try to identify the nonlinearity by assuming appropriate function. Based on the experimental results, $F_{M}$ can be considered as following

$$
F_{M}=\alpha_{0} w_{r e l}+\alpha_{n} w_{r e l}^{3}
$$

where $\alpha_{0}$ and $\alpha_{n}$ are two unknowns which can be obtained by using experimental results. In order to find the dynamic response of the beam, the deflection of the beam is represented as a series expansion in terms of the eigenfunctions of the beam, i.e.

$$
w_{r e l}(x, t)=\sum_{i=1}^{N} U_{i}(t) \varphi_{i}(x)
$$

Using the Galerkin method, equation (5.1) can be converted into a system of differential equations. Considering a single-mode approximation yields the following equation

$$
M \ddot{U}(t)+C \dot{U}(t)+\left(K_{m}-K_{0}\right) U(t)-K_{n} U^{3}=F_{b} \cos (\Omega t)
$$

where

$$
\begin{aligned}
& M=\int_{0}^{L}\left(m+M_{s} \delta\left(x-L_{s}\right)\right) \varphi^{2}(x) d x, \quad K_{0}=\alpha_{0} \int_{0}^{L} \varphi^{2}(x) \delta(x-L) d x \\
& K_{m}=E I \int_{0}^{L} \varphi(x) \varphi^{I V}(x) d x, \quad C=c_{a} \int_{0}^{L} \varphi^{2}(x) d x
\end{aligned}
$$




$$
\begin{aligned}
& K_{n}=\alpha_{n} \int_{0}^{L} \varphi^{4}(x) \delta(x-L) d x \\
& F_{b}=z_{0} \Omega^{2}\left(\int_{0}^{L}\left(m+M_{s} \delta\left(x-L_{s}\right)+M_{t} \delta(x-L)\right) \varphi(x) d x\right)
\end{aligned}
$$

For convenience, equation (5.5) can be rewritten as

$$
\ddot{U}(t)+2 \mu \omega_{n} \dot{U}(t)+\omega_{n}^{2} U(t)-\beta_{n} U^{3}(t)=F \cos (\Omega t)
$$

where

$$
\mu=\frac{C}{2 M \omega_{n}}, \quad \omega_{n}=\sqrt{\frac{K_{m}-K_{0}}{M}}, \quad F=\frac{F_{b}}{M}, \quad \beta_{n}=\frac{K_{n}}{M}
$$

To determine the analytical solution of the transverse vibration of the beam, the harmonic balance method is used. By assuming a steady state periodic response, $U(t)$ and $v_{p}(t)$ can be written as

$$
U(t)=a_{1}(t) \sin (\Omega t)+b_{1}(t) \cos (\Omega t)
$$

with slowly varying coefficients such that

$$
\begin{gathered}
\dot{U}(t)=\left(\dot{b}_{1}+a_{1} \Omega\right) \cos (\Omega t)+\left(\dot{a}_{1}-b_{1} \Omega\right) \sin (\Omega t) \\
\ddot{U}(t)=\Omega\left(2 \dot{a}_{1}-b_{1} \Omega\right) \cos (\Omega t)-\Omega\left(2 \dot{b}_{1}+a_{1} \Omega\right) \sin (\Omega t)
\end{gathered}
$$

Substituting the above expressions into equation (5.7), neglecting higher harmonics and balancing terms multiplied by $\sin (\Omega t)$ and $\cos (\Omega t)$, from the mechanical equation the following equations are obtained

$$
\begin{gathered}
2 \mu \omega_{n} \dot{a}_{1}-2 \Omega \dot{b}_{1}=Q a_{1}+2 \mu \omega_{n} b_{1} \Omega \\
2 \mu \omega_{n} \dot{b}_{1}+2 \Omega \dot{a}_{1}=Q b_{1}+F-2 \mu \omega_{n} a_{1} \Omega
\end{gathered}
$$

where

$$
r^{2}=a_{1}^{2}+b_{1}^{2}
$$




$$
Q=-\omega_{n}^{2}+\Omega^{2}-\frac{3}{4} \alpha r^{2}
$$

In a steady state, all time derivatives vanish so that the mechanical amplitude equations can be rewritten as

$$
\begin{aligned}
& Q a_{1}+2 \mu \omega_{n} b_{1} \Omega=0 \\
& 2 \mu \omega_{n} a_{1} \Omega-Q b_{1}=F
\end{aligned}
$$

squaring and adding equations (3.29) and (3.30) gives a sixth order nonlinear algebraic equation in $r$ as

$$
F^{2}=r^{2}\left(Q^{2}+\left(2 \mu \omega_{n} \Omega\right)^{2}\right)
$$

and the frequency response can be determined by numerically finding the positive real roots of equation (5.18). To validate the developed mathematical model with experimental results, firstly the linear behaviour of the beam is studied. As shown in Figure 5.13, there is a good agreement between the dynamic results obtained by experiment and the theory.

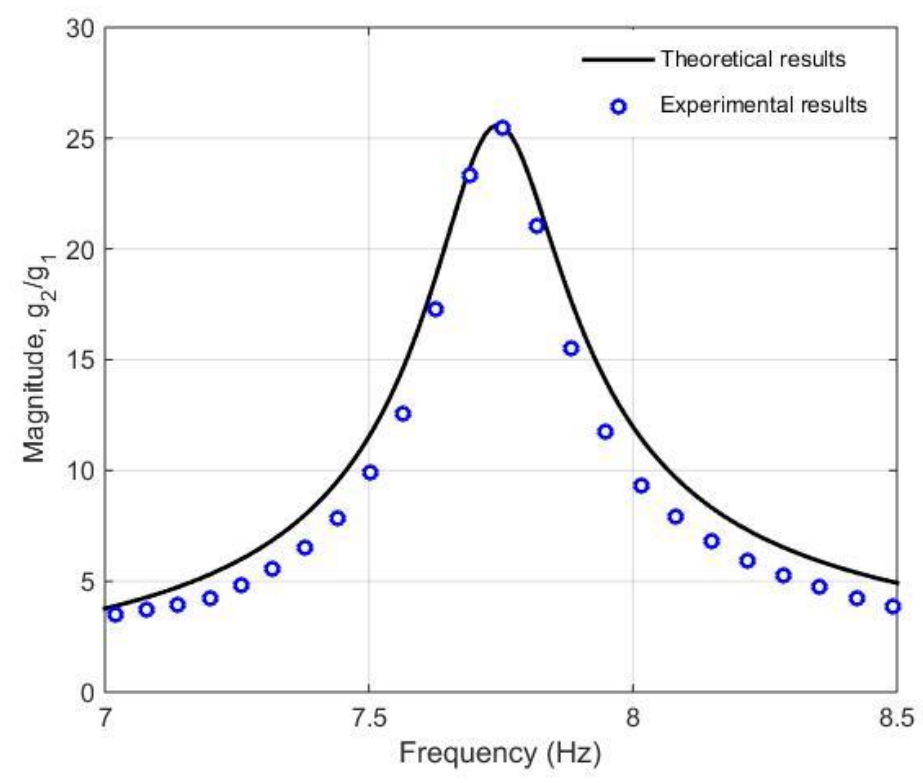

Figure 5.13 FRF of the linear model: comparison between experimental and theoretical results 
In order to validate the nonlinear behaviour of the proposed model, it is necessary to find the unknown linear and nonlinear stiffness $\left(K_{0}\right.$ and $\left.K_{n}\right)$, which is related to the magnetic force. To this end, Matlab optimization toolbox is used to match the resonance frequencies for different scenario. Figure 5.14 shows the results for the attractive force configuration. As shown in this figure, by choosing appropriate $K_{0}$ and $K_{n}$, the theoretical model matches the experimental results.

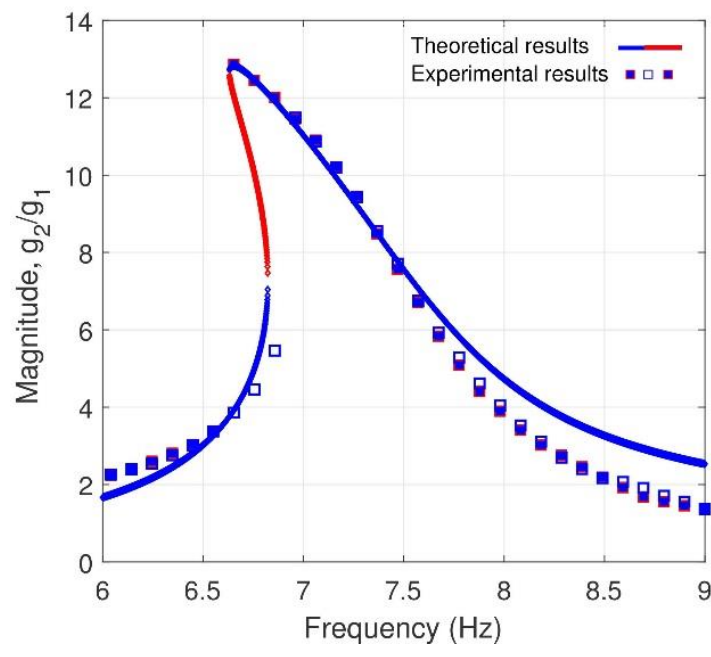

(a) $g_{0}=25 \mathrm{~mm}$

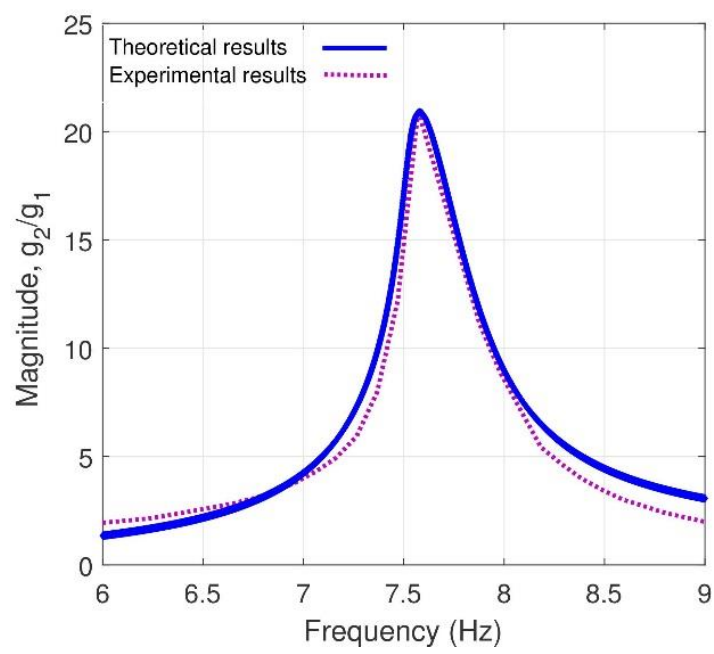

(c) $g_{0}=35 \mathrm{~mm}$

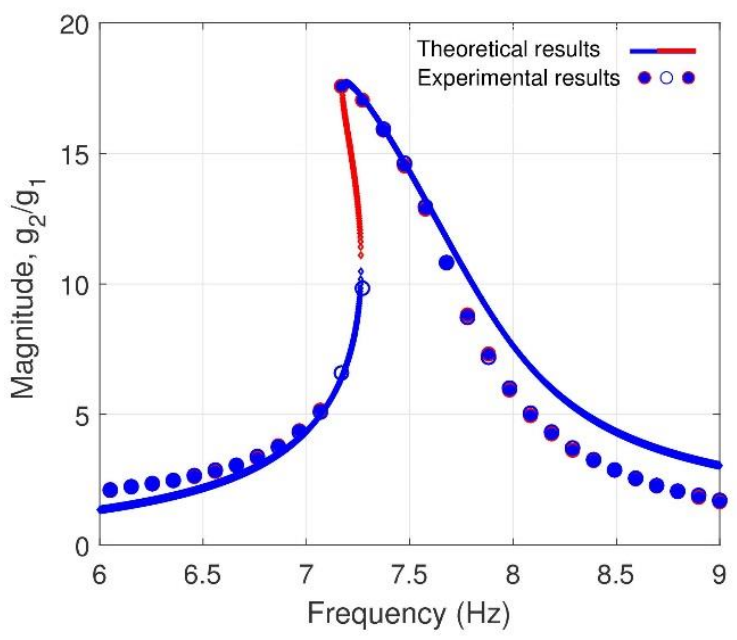

(b) $g_{0}=30 \mathrm{~mm}$

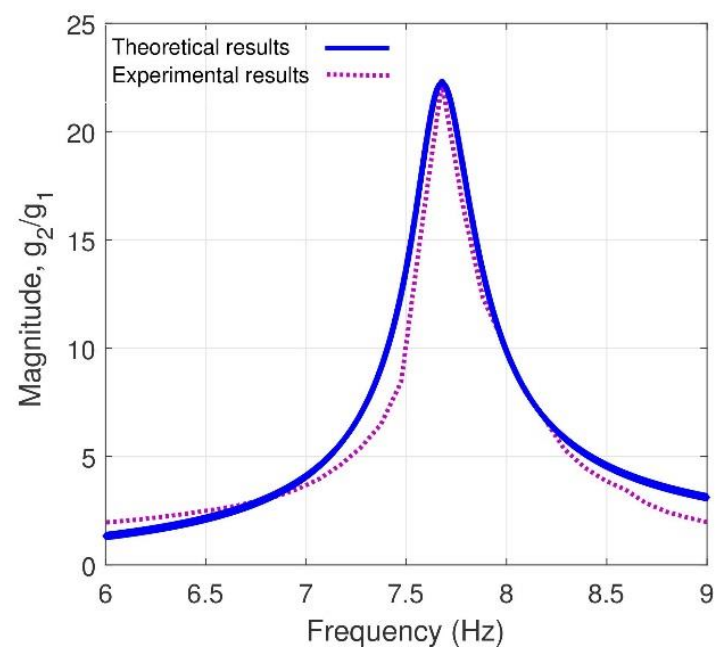

(d) $g_{0}=40 \mathrm{~mm}$

Figure 5.14 FRF of the beam for different air gaps between magnets (there is no applied voltage to the electromagnets)

It can be seen that for each initial gap, new values for $K_{0}$ and $K_{n}$ are obtained. Table 5.2 shows the values of $K_{0}$ and $K_{n}$ for different initial gaps. As shown in Table 5.2, increasing 
the initial gap decreases the magnetic nonlinearity and, consequently, $K_{0}$ and $K_{n}$ decrease. On the other hand, by applying voltage to the electromagnets, the magnetic nonlinearity increases. Considering $30 \mathrm{~mm}$ as the initial gap between electrodes in the attractive force configuration, Figure 5.15 compares the results of the experiment with the theoretical model in four cases where DC voltage applied to the electromagnets.

Table 5.2 Identified values for $K_{0}$ and $K_{n}$ for different initial gaps at $V=0 \mathrm{~V}$

\begin{tabular}{|c|c|c|} 
& $\boldsymbol{K}_{\mathbf{0}}$ & $\boldsymbol{K}_{\boldsymbol{n}}$ \\
\hline $\boldsymbol{g}_{\mathbf{0}}=\mathbf{2 5} \mathbf{m m}$ & 2.016556072496814 & $1.586798007393011 \mathrm{e} 4$ \\
\hline $\boldsymbol{g}_{\mathbf{0}}=\mathbf{3 0} \mathbf{m m}$ & 1.065831847069356 & $5.724431999224028 \mathrm{e} 3$ \\
\hline $\boldsymbol{g}_{\mathbf{0}}=\mathbf{3 5} \mathbf{~ m m}$ & 0.946488016069645 & $7.473075848312722 \mathrm{e} 2$ \\
\hline $\boldsymbol{g}_{\mathbf{0}}=\mathbf{4 0} \mathbf{m m}$ & 0.095689938424641 & $5.0368531217627762 \mathrm{e} 1$ \\
\hline
\end{tabular}

As shown in Figure 5.15, the nonlinear behaviour of the beam can be modelled by the assumed function for the cases in which voltage is applied. However, as Figure 5.15 shows by increasing applied DC voltage to the electromagnets the discrepancy between theoretical and experimental curves increases. This behaviour can be related to the use of approximated function for the electromagnetic nonlinearity. In order to get more accurate results we need use more precise function by adding more nonlinear terms to our approximation. The values for $K_{0}$ and $K_{n}$ have been shown in Table 5.3. The comparison between the experimental results and the proposed theoretical model shows that the nonlinear behaviour of the beam can be modelled with the assumed function for the magnetic force. 


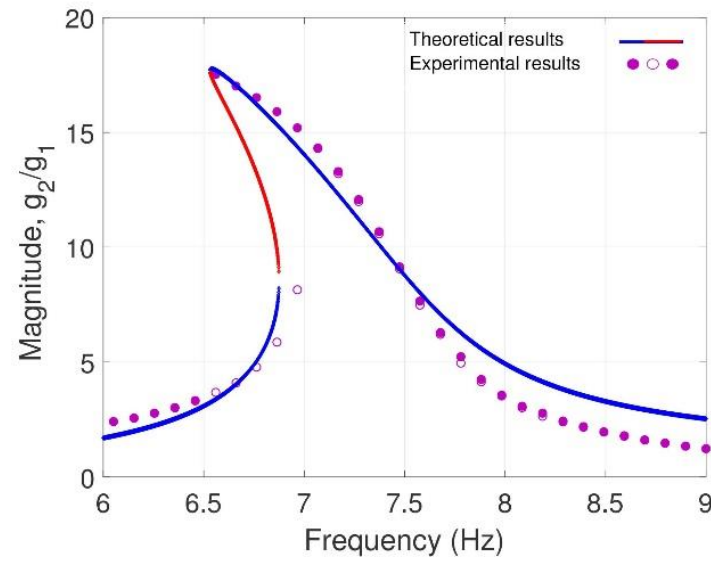

(a) $V=4 V$

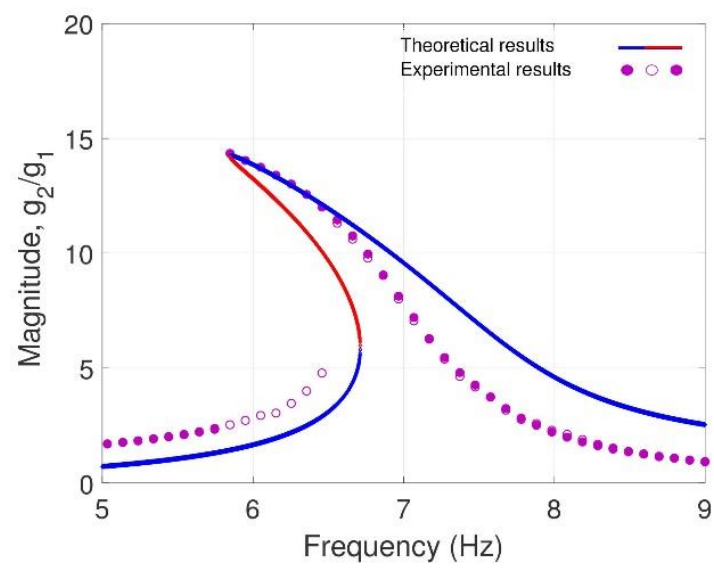

(c) $V=12 \mathrm{~V}$

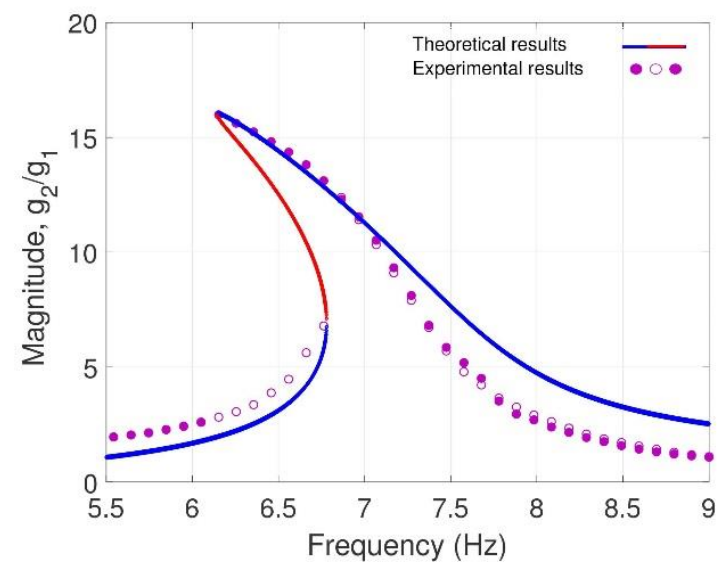

(b) $V=8 \mathrm{~V}$

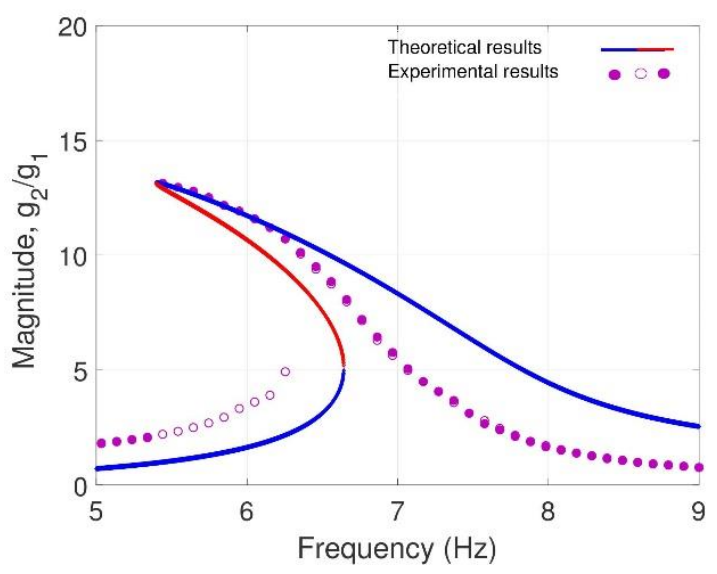

(d) $V=16 V$

Figure 5.15 FRF of the beam $\left(g_{0}=30 \mathrm{~mm}\right)$

However, by increasing the voltage applied to the electromagnets, the margin of error increases and more precise function may be needed to model magnetic nonlinearity.

Table 5.3 Identified values for $K_{0}$ and $K_{n}$ for different voltages at $g_{0}=30 \mathrm{~mm}$

\begin{tabular}{|c|c|c|}
\hline & \multicolumn{1}{|c|}{$\boldsymbol{K}_{\mathbf{0}}$} & $\boldsymbol{K}_{\boldsymbol{n}}$ \\
\hline $\boldsymbol{V}=\mathbf{0} \boldsymbol{V}$ & 1.065831847069356 & $5.724431999224028 \mathrm{e} 3$ \\
\hline $\boldsymbol{V}=\mathbf{4} \boldsymbol{V}$ & 1.172415031776292 & $9.159091198758446 \mathrm{e} 3$ \\
\hline $\boldsymbol{V}=\mathbf{8} \boldsymbol{V}$ & 1.598747770604034 & $1.630840959782728 \mathrm{e} 4$ \\
\hline $\boldsymbol{V}=\mathbf{1 2} \boldsymbol{V}$ & 2.664579617673390 & $2.560171196818515 \mathrm{e} 4$ \\
\hline $\boldsymbol{V}=\mathbf{1 6} \boldsymbol{V}$ & 3.730411464742746 & $3.809035195266570 \mathrm{e} 4$ \\
\hline $\boldsymbol{V}=\mathbf{2 0} \boldsymbol{V}$ & 4.796243311812102 & $4.933012793869821 \mathrm{e} 4$ \\
\hline
\end{tabular}




\subsection{Energy harvester}

In this section, the dynamic behaviour of the proposed harvester is studied. To this end, the MFC piezoelectric patches (M8585-P2) were bonded to the beam. The geometric and other properties of the piezoelectric patches have been listed in Table 5.4.

As shown in Figure 5.16, in this case lightweight ICP sensors were used to measure the acceleration of the beam and base.

Table 5.4 Geometrical and material properties of the MFC piezoelectric patches

\begin{tabular}{lc}
\hline Design Variable & Value \\
\hline Overall length, $L_{\tilde{p}}(\mathrm{~mm})$ & 103 \\
Overall width, $b(\mathrm{~mm})$ & 31 \\
Thickness, $h_{\tilde{p}}(\mathrm{~mm})$ & 0.3 \\
Young's modulus, $E_{\tilde{p}}(\mathrm{GPa})$ & 30.336 \\
Density of beam, $\rho_{\tilde{p}}\left(\mathrm{~kg} / \mathrm{m}^{3}\right)$ & 7740 \\
Piezoelectric coefficient, 31-mode $\left(d_{31}\right)$ & $-2.1 \mathrm{e} 2 \mathrm{pC} / \mathrm{N}$ \\
Piezoelectric coefficient, 33-mode $\left(d_{33}\right)$ & $4.0 \mathrm{e} 2 \mathrm{pC} / \mathrm{N}$
\end{tabular}

Adding piezoelectric patches to the beam increases the resonance frequency of the beam. Using a random burst excitation, the first three resonance frequencies of the beam were identified. As Figure 5.17 shows, the first, second and third resonance frequencies of the beam are $9.2 \mathrm{~Hz}, 54.1 \mathrm{~Hz}$ and $152.8 \mathrm{~Hz}$, respectively.

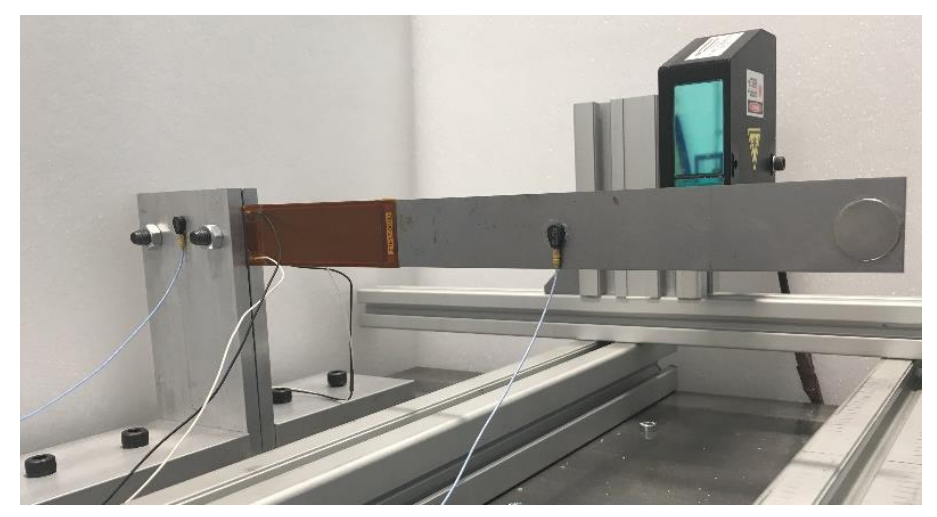

Figure 5.16 Proposed energy harvester

Afterwards, the close circuit condition was investigated for the harvester. Due to the input voltage limitation of the SignalCalc analyser a voltage divider circuit was designed to satisfy the input voltage condition of the SignalCalc analyser. As Figure 5.18 shows, the voltage divider circuit consist of two resistors $\left(R_{1}=10.04 M \Omega\right.$ and $\left.R_{2}=98.8 \mathrm{k} \Omega\right)$ in 
series connection which was connected to the $\mathrm{AC}$ current coming from the piezoelectric patches.

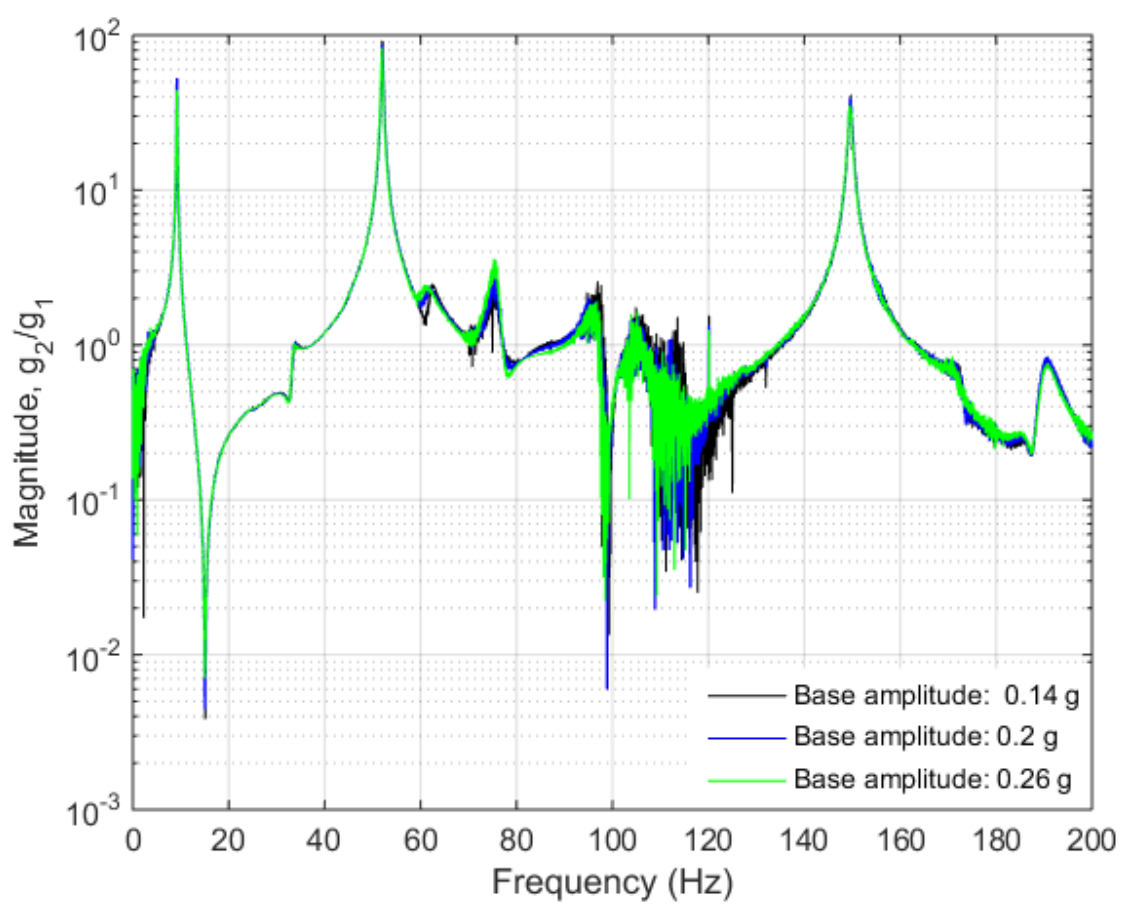

Figure 5.17 FRFs of the linear model with added MFC piezoelectric patches

Generally, a voltage divider circuit takes a higher voltage and converts it to a lower one by using a pair of resistors. The formula for calculating the output voltage is based on Ohms Law and is expressed as follows

$$
V_{2}=\frac{V_{p} R_{2}}{\left(R_{1}+R_{2}\right)}
$$

where $V_{p}$ is the output voltage of the piezoelectric patches. The connection of the piezoelectric patches can be either series or parallel depending on the application. Here we have considered parallel connection. However, as first step we measured the output voltage of each patch separately to make sure they generated the same voltage. 


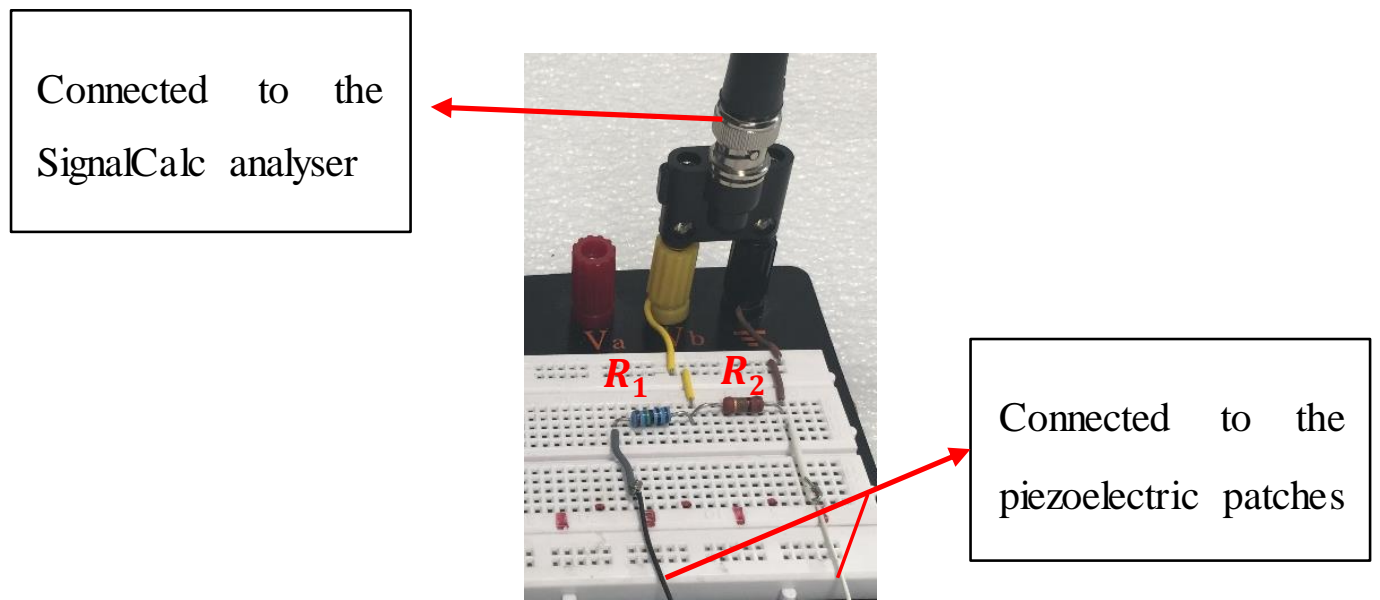

Figure 5.18 voltage divider circuit

As shown in Figure 5.18, both patches gave almost the same output voltage. There is a negligible difference in the peak, which can be related to wiring losses and differences in the thickness of the adhesive layer on both sides. It is noticeable that the measured voltage is related to the voltage cross smaller resistor $\left(R_{2}\right)$. Figure 5.19 illustrates that $3.76 \mathrm{~V} / \mathrm{g}$ can be obtained at the resonance frequency: considering this value and using equation (5.21), the total output voltage of the piezoelectric patch $\left(V_{p}\right)$ is $385.849 \mathrm{~V} / \mathrm{g}$.

Using the frequency tuning mechanism explained in the previous section, the resonance frequency of the harvester can be tuned to harvest more power.

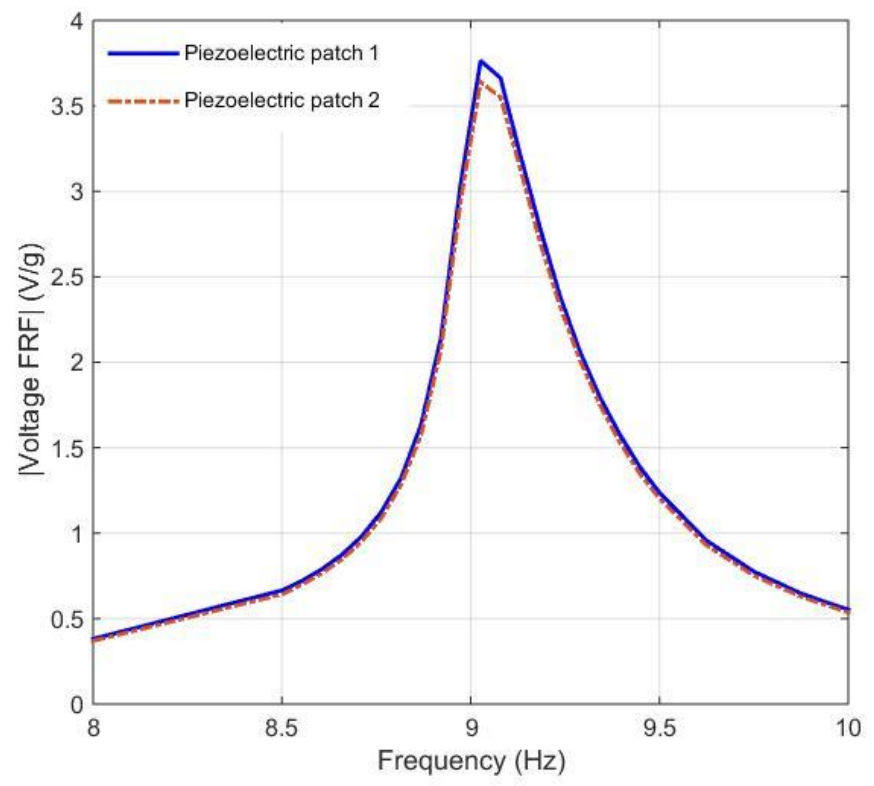

Figure 5.19 Voltage output FRFs of the piezoelectric patches. 
The experimental set-up including the tuning mechanism has been shown in Figure 5.19. Considering $25 \mathrm{~mm}$ as an initial gap between magnets, Figure 5.20 shows the scenario in which $5 \mathrm{~V}$ was applied to the electromagnets in the attractive configuration.

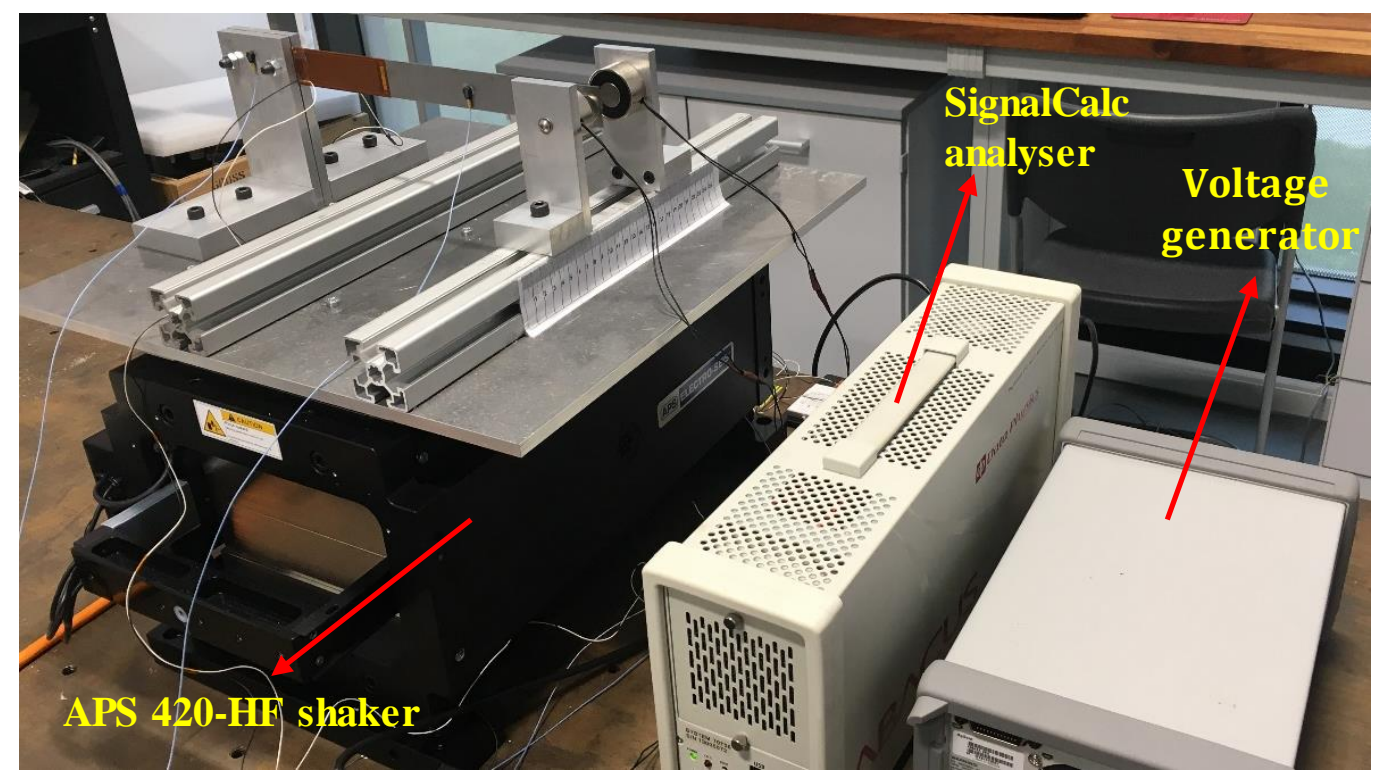

Figure 5.20 Proposed energy harvester with frequency tuning mechanism

As shown in Figure 5.20, by applying $5 \mathrm{~V}$ to the electromagnets, the resonance frequency of the harvester can be decreased to $7.24 \mathrm{~Hz}$, and $2.83 \mathrm{~V} / \mathrm{g}$ can be obtained at the resonance frequency. Here we need to consider the sustainability of the harvester by comparing the current generated and consumed to change the resonance frequency.

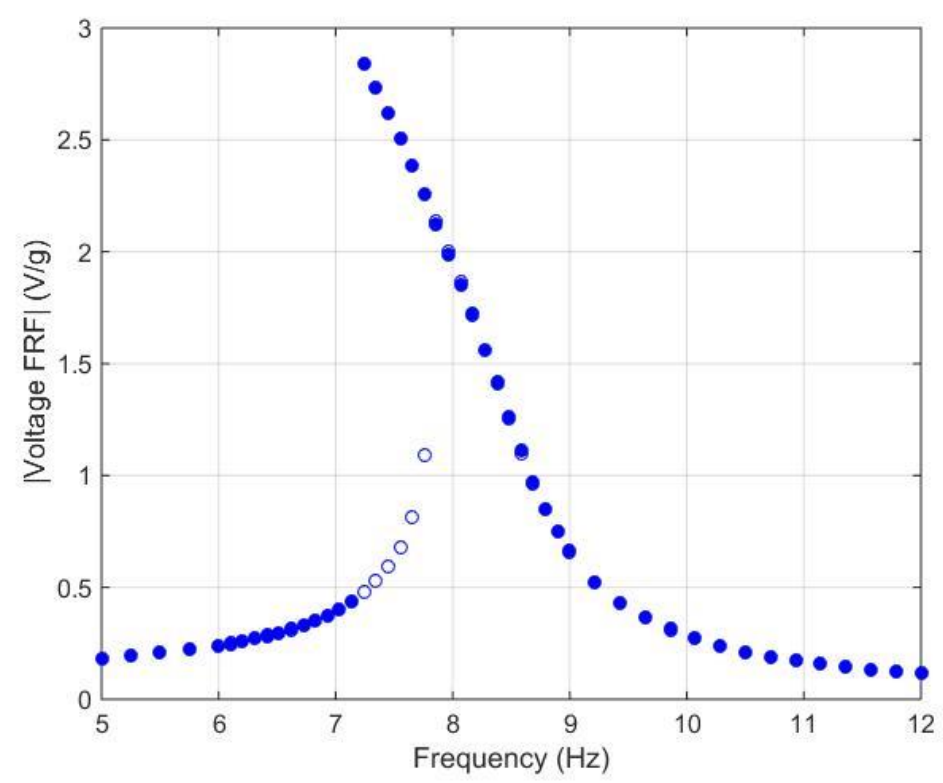

Figure 5.21 Voltage output FRF of the piezoelectric patches when $V_{D C}=5 \mathrm{~V}$ 
Using equation (5.21), the total output voltage of the piezoelectric patches $\left(V_{p}\right)$ is 290.41 V/g. Considering the equivalent resistor $\left(R_{e q}\right)$ of the voltage divider circuit and using Ohm's law, the current generated by the harvester $\left(I_{p}\right)$ can be obtained as follows

$$
I_{p}=\frac{V_{p}}{R_{e q}}=\frac{290.41}{10.13 \times 10^{6}}=0.028 \mathrm{~mA} / \mathrm{g}
$$

Whereas the voltage generator uses $0.113 \mathrm{~A}$ to provide $5 \mathrm{~V}$ to the electromagnets. By comparing the input current with the output current, we find that the proposed harvester is not sustainable. However, by considering the movements of the tip magnets we can take the advantage of electromagnetic harvesters and modify our model to a hybrid piezoelectric and electromagnetic harvester. As discussed in Chapter one, by changing the magnetic flux across a coil the electrical current will be induced. Therefore, by adding coils around movable magnets the output power of the harvester can be improved. 


\subsection{Closure}

In this chapter, an experimental set-up was designed to tune the resonance frequency of the beam using magnetic forces, which have similar nonlinearity to electrostatic forces. It has been shown that depending on the arrangement of the magnet poles the resonance frequency of the beam can be either decreased or increased. Based on the experimental results, with the magnets in the attractive force configuration, increasing the magnetic force decreases the resonance frequency of the beam through softening nonlinearity, whereas in the repulsive force configuration, it can be increased. Different initial gaps between magnets were considered and showed that with a $25 \mathrm{~mm}$ air gap between magnets and by increasing the voltage applied to the electromagnets up to $20 \mathrm{~V}$ in both attractive and repulsive force configurations, the resonance frequency of the beam can be changed from 3.8 to $9.1 \mathrm{~Hz}$.

In order to verify the experimental results, an odd polynomial function up to the third order was considered for the magnetic force. The steady-state solution was obtained using the harmonic balance method and it has been shown that the resonance frequency obtained by the theoretical model matches the experimental results. However, it also showed that increasing the voltage applied to the electromagnets increases the margin of error between the experimental and theoretical results and more precise function may be needed to model magnetic nonlinearity.

In addition, the use of the proposed electromagnetic tuning mechanism for energy harvesting applications was studied. To this end, MFC piezoelectric patches were bonded to the beam and the sustainability of the energy harvester was investigated. The results showed that the proposed harvester is not sustainable. However it can be upgraded to a hybrid piezoelectric and electromagnetic harvester to increase the efficiency of the harvester. 


\section{Chapter 6: Conclusions and future work}

This chapter summarises the conclusions derived from the previous chapters and highlights the future directions of the work related to this thesis.

\section{Conclusions}

In this thesis, dynamic behaviour of vibration-based MEMS piezoelectric harvesters have been studied and the effect of manufacturing uncertainties in the performance of MEMS piezoelectric harvesters have been investigated.

In the first chapter of this thesis, a brief overview of microelectromechanical systems (MEMS) was given and the importance of using energy harvesting technology to provide power for MEMS devices was highlighted. A comprehensive review was carried out to establish awareness of the different MEMS vibration-based energy harvesters proposed. Then, in the second chapter, a reduced-order model was developed to study the dynamic behaviour of MEMS piezoelectric harvesters.

An arbitrary bimorph MEMS piezoelectric harvester was considered, and it was shown that depending on the load resistance connected to the piezoelectric layers, the resonance frequency of the harvetser can be changed between short-circuit and open-circuit frequencies. The effect of different parameters in the resonance frequency shift were discussed and based on the obtained results, it was observed that the resonance frequency shift $\left(\Delta \omega_{r}=\omega_{r}^{o c}-\omega_{r}^{s c}\right)$ is mostly related to the effect of the piezoelectric layers and electrical parameters. Furthermore, the results showed that by increasing load resistance, the output volatge increases monotonically wheras the amplitude of vibration is decreased in the short-circuit condition (original resonance frequency) and amplified considerably at the open-circuit resonance frequency. 
In addition, it was shown that the output power of the harvester can be maximised by using the optimal resisctance. However, any mismatch between the resonance frequency of the harvester and the excitation frequency can decrease the harvested power significantly. To overcome this problem, in the third chapter a MEMS piezoelectric harvester with an adjustable resonance frequency was proposed. It was shown that by using an electrostatic device, the resonance frequency of the harvester can be decreased to harvest power in a wide range of excitaion frquencies.

The effect of different parameters on the performance of the proposed harvester were invesigated and a MEMS piezoelectric harvester was designed for a vibration source with a frequency range of $70-110 \mathrm{~Hz}$ and $0.25 \mu \mathrm{m}$ amplitude of base excitation. The disadvantage of the model proposed in Chapter three was the effect of the softening nonlinearity of the electrostatic part of the harvester which resulted in a lower level of harvested energy.

In Chapter four, different types of uncertain parameters in the design of a MEMS piezoelectric harvester were studied and the results showed that manufacturing uncertainty could potentially change the harvester's resonance frequency and, consequently, the deviation from its nominal value may be positive or negative. Therefore, there is a need to tune the harvester's resonance frequency to a higher (hardening) or lower (softening) frequency. In order to tackle this problem, the electrostatic tuning device was improved by adding the capability of increasing the resonance frequency of the samples via a hardening mechanism. In order to show the results, experimentally measured statistical properties available in the literature were considered and Monte Carlo Simulation (MCS) was used for uncertainty propagation.

The problem that we encountered in the fourth chapter was a nonlinear uncertain dynamic problem which is a very challenging problem and there is little attention to it in the literature. It was found that the use of semi-analytical solutions and time integration is not feasible. Therefore, the combined use of a shooting method and Monte Carlo Simulation was used. The method was found to be efficient enough to solve this uncertain and nonlinear problem.

The sustainability of the proposed model was also investigated and it has been shown that the power losses of voltage sources in both mechanisms are negligible. Therefore, the proposed model can be used in real-life applications. Due to the lack of access to MEMS 
fabrication facilities, the concept of the tuning mechanism was shown in large scale in Chapter five. To this end, an experimental set up was designed to tune the resonance frequency of the beam using magnetic forces. It was shown that by using an electromagnetic device the resonance frequency of the beam can be either decreased or increased. The experimental results showed that by using the electromagnetic device in the attractive force configuration, the resonance frequency of the beam can be decreased through the softening nonlinearity, whereas in the repulsive force configuration, it can be increased. For an arbitrary case of using the electromagnetic device, it was shown that by applying voltage to the electromagnets up to $20 \mathrm{~V}$ in both attractive and repulsive force configurations, the resonance frequency of the beam can be changed from 3.8 to $9.1 \mathrm{~Hz}$.

A mathematical model was developed to verify the experimental results, and it has been shown that the resonance frequency obtained by the theoretical model matches the experimental results. However, it was also showed that increasing the voltage applied to the electromagnets increases the margin of error between the experimental and the theoretical results and more precise function may be needed to model magnetic nonlinearity. The use of the electromagnetic tuning mechanism in energy harvesting applications was studied. The results showed that using an electromagnetic device to tune the resonance frequency of the piezoelectric harvester is not efficient. However, to increase its efficiency the model can be modified to a hybrid piezoelectric and electromagnetic harvester.

\section{Suggestions for future work}

Future work related to this thesis can involve improving the electrostatic device by modifying the tuning mechanisms. In the current proposed model using the softening nonlinearity of the electrostatic part results in a lower level of harvested energy. On the other hand, the hardening mechanism may have some fabrication difficulties. Therefore, finding new sustainable tuning mechanisms can be considered as a potential future work.

Considering the nonlinearity of the electrostatic force and uncertainty at the same time, future work may focus on finding more efficient methods to deal with nonlinear uncertain problem. In addition, it may involve in investigating the role of global sensitivity analysis 
in the selection of uncertain parameters, and the robust design of such a system to passively minimise the adverse effects of uncertainty in the harvester.

The identification of the electromagnetic nonlinear force requires fusther studies where the analysis presented in this work was not able to provide the best match between expemental data and the theoritical results. 


\section{Appendix A}

Coefficients of equation (3.9) are as following

$$
\begin{array}{ll}
a_{1}=\frac{-2 \varepsilon_{0} b V_{D C}^{2}}{g_{0}^{3}}, & a_{2}=\frac{-4 \varepsilon_{0} b V_{D C}^{2}}{g_{0}^{5}}, \quad a_{3}=\frac{-6 \varepsilon_{0} b V_{D C}^{2}}{g_{0}^{7}} \\
a_{4}=\frac{-8 \varepsilon_{0} b V_{D C}^{2}}{g_{0}^{9}}, & a_{5}=\frac{-10 \varepsilon_{0} b V_{D C}^{2}}{g_{0}^{11}}
\end{array}
$$

Coefficients of equation (4.9) and (4.10) are as following

$$
\begin{aligned}
& c_{a}=\frac{\hat{c}_{a} L^{4}}{(E I)_{C} T}, \quad T=\sqrt{\frac{m_{C} L^{4}}{E I_{C}}}, \quad \vartheta_{p}\left(\psi_{4}\right)=\frac{\hat{\vartheta}_{p} L^{2}}{E I_{C} g_{01}}, \\
& \alpha_{f}\left(\psi_{3}\right)=\frac{\varepsilon_{0} a L^{3} \alpha}{E I_{C} g_{r}^{2}}, \quad \alpha_{e}\left(\psi_{5}\right)=\frac{\varepsilon_{0} a L^{4}}{2 g_{01}^{3} E I_{C}}, \quad \sigma_{1}=\frac{z_{0} L^{4} m_{C}}{T^{2} g_{01} E I_{C}} \\
& \sigma_{2}=\frac{z_{0} L^{3} M_{t}}{T^{2} g_{01} E I_{C}}, \quad \lambda\left(\psi_{7}\right)=\frac{T}{2 R C_{p}}, \quad \gamma\left(\psi_{8}\right)=\frac{\bar{e}_{31} a g_{01}\left(h_{0}+h\right)}{2 C_{p} L} \\
& r\left(\psi_{6}\right)=\frac{g_{02}}{g_{01}}, \quad s_{1}\left(x, \psi_{1}\right)=\frac{E I\left(\hat{x}, \hat{\psi}_{1}\right)}{E I_{C}}, \quad s_{2}\left(x, \psi_{2}\right)=\frac{m\left(\hat{x}, \hat{\psi}_{2}\right)}{m_{C}}
\end{aligned}
$$

Coefficients of equation (4.10) and (4.11) are as following

$$
\begin{aligned}
& M=\int_{0}^{1} s_{2}\left(x, \psi_{2}\right) \varphi^{2}(x) d x, \quad C=c_{a} \int_{0}^{1} \varphi^{2}(x) d x, \\
& K_{f}=\alpha_{f} V_{2}^{2} \int_{0}^{1} \varphi(x) \varphi^{I I}(x) d x, \quad \theta_{p}=\frac{\vartheta_{p}}{M}\left(\frac{d \varphi\left(l_{c}\right)}{d x}\right) \\
& K_{m}=\int_{0}^{1}\left(s_{1}^{I I}\left(x, \psi_{1}\right) \varphi^{I I}(x)+2 s_{1}{ }^{I}\left(x, \psi_{1}\right) \varphi^{I I I}(x)\right. \\
& \left.\quad+s_{1}\left(x, \psi_{1}\right) \varphi^{I V}(x)\right) \varphi(x) d x, \\
& \alpha_{t}=\frac{1}{M}\left(\sigma_{1} \int_{0}^{1} \varphi(x) s_{2}\left(x, \psi_{2}\right) d x+\sigma_{2} \int_{0}^{1} \varphi(x) \delta(x-1) d x\right), \\
& \beta=\gamma\left(\frac{d \varphi\left(l_{c}\right)}{d x}\right), \quad \mu=\frac{C}{2 M \omega_{n}}, \quad \omega_{n}=\sqrt{\frac{K_{m}+K_{f}}{M}},
\end{aligned}
$$




\section{References}

[1] Seok S. Overview of MEMS Packaging Technologies. Advanced Packaging and Manufacturing Technology Based on Adhesion Engineering: Wafer-Level Transfer Packaging and Fabrication Techniques Using Interface Energy Control Method. Cham: Springer International Publishing; 2018. p. 1-12.

[2] Velosa-Moncada LA, Aguilera-Cortés LA, González-Palacios MA, Raskin J-P, Herrera-May AL. Design of a Novel MEMS Microgripper with Rotatory Electrostatic Comb-Drive Actuators for Biomedical Applications. Sensors. 2018;18(5):1664.

[3] Tuominen J, Lehtonen E, Tadi MJ, Koskinen J, Pänkäälä M, Koivisto T. A miniaturized low power biomedical sensor node for clinical research and long term monitoring of cardiovascular signals. Conference A miniaturized low power biomedical sensor node for clinical research and long term monitoring of cardiovascular signals. IEEE, p. 1-4.

[4] Mileshin S, Tsivinskaya T, Sergeeva N. Microelectromechanical sensors and microstructures in aerospace applications. KnE Engineering. 2017;2(3).

[5] Yuan W. Development and application of high-end aerospace MEMS. Frontiers of Mechanical Engineering. 2017;12(4):567-73.

[6] Hiller T, Kuhlmann B, Buhmann A, Roth H. Noise contributions in a closed-loop MEMS gyroscope for automotive applications. Conference Noise contributions in a closed-loop MEMS gyroscope for automotive applications. IEEE, p. 62-5.

[7] Zachäus C, Müller B, Meyer G. Advanced Microsystems for Automotive Applications 2017: Smart Systems Transforming the Automobile: Springer, 2017.

[8] Yuan Q, Kan X, Chen Z, Yang J, Zhao J, Sun L, et al. RF MEMS resonant devices for wireless communication. Conference RF MEMS resonant devices for wireless communication. IEEE, p. 1-4.

[9] Lee EK, Kim CS, Kim JJ, Choi HR. A study on the fabrication and evaluation of the MEMS based FBAR filter for wireless systems. Conference A study on the fabrication and evaluation of the MEMS based FBAR filter for wireless systems. IEEE, p. 21-4. 
[10] Maroufi M, Ruppert MG, Fowler AG, Moheimani SR. Design and control of a single-chip SOI-MEMS atomic force microscope. Conference Design and control of a single-chip SOI-MEMS atomic force microscope. IEEE, p. 2869-74.

[11] Dziekoński C, Dera W, Jarząbek DM. Method for lateral force calibration in atomic force microscope using MEMS microforce sensor. Ultramicroscopy. 2017;182:1-9.

[12] Haikka P, Kubo Y, Bienfait A, Bertet P, Mølmer K. Proposal for detecting a single electron spin in a microwave resonator. Physical Review A. 2017;95(2):022306.

[13] Vabret N, Bhardwaj N, Greenbaum BD. Sequence-specific sensing of nucleic acids. Trends in immunology. 2017;38(1):53-65.

[14] Soysal U, Marty F, Algré E, Géhin E, Motzkus C. Sub- $\mu$ m air-gap resonant MEMS mass sensors fabrication and electrical characterization for the detection of airborne particles. Conference Sub- $\mu \mathrm{m}$ air-gap resonant MEMS mass sensors fabrication and electrical characterization for the detection of airborne particles. IEEE, p. 1-5.

[15] Keeler EG, Jing P, Wu J, Zou C, Lin LY. MEMS Resonant Mass Sensor Integrated with Optical Manipulation. IEEE Transactions on Nanotechnology. 2018.

[16] Gupta A, Singh TS, Yadava R. Polymer-coated MEMS chemical sensor array for monitoring oxidative stress by breath analysis. Conference Polymer-coated MEMS chemical sensor array for monitoring oxidative stress by breath analysis. IEEE, p. 1-8.

[17] Wittmeier F, Kuthada T, Filipsky J, Cizek J. New MEMS Pressure Sensors for Transient Aerodynamic Measurements. ATZ worldwide. 2018;120(4):38-41.

[18] Hasan MH, Alsaleem FM, Ouakad HM. Novel threshold pressure sensors based on nonlinear dynamics of MEMS resonators. Journal of Micromechanics and Microengineering. 2018;28(6):065007.

[19] Status of the MEMS 2014. Yole Développement. (Villeurbanne, France).

[20] Williams CB, Yates RB. Analysis of a micro-electric generator for microsyste ms. Sensors and Actuators A: Physical. 1996;52(1):8-11. 
[21] Roundy S, Wright PK, Rabaey J. A study of low level vibrations as a power source for wireless sensor nodes. Computer communications. 2003;26(11):1131-44.

[22] Jeon Y, Sood R, Jeong J-H, Kim S-G. MEMS power generator with transverse mode thin film PZT. Sensors and Actuators A: Physical. 2005;122(1):16-22.

[23] Glynne-Jones P, Tudor MJ, Beeby SP, White NM. An electromagnetic, vibrationpowered generator for intelligent sensor systems. Sensors and Actuators A: Physical. 2004;110(1):344-9.

[24] Arnold DP. Review of microscale magnetic power generation. IEEE Transactions on Magnetics. 2007;43(11):3940-51.

[25] Roundy S, Wright PK, Rabaey JM. Energy scavenging for wireless sensor networks. Norwell. 2003.

[26] Mitcheson PD, Miao P, Stark BH, Yeatman E, Holmes A, Green T. MEMS electrostatic micropower generator for low frequency operation. Sensors and Actuators A: Physical. 2004;115(2):523-9.

[27] Nye JF. Physical properties of crystals: their representation by tensors and matrices: Oxford university press, 1985.

[28] Yang B, Liu H, Liu J, Lee C. Micro and Nano Energy Harvesting Technologies: Artech House, 2014.

[29] Gonzalo JA, Jiménez B. Ferroelectricity: The Fundamentals Collection: John Wiley \& Sons, 2008.

[30] Anton SR, Sodano HA. A review of power harvesting using piezoelectric materials (2003-2006). Smart materials and Structures. 2007;16(3):R1.

[31] Aboulfotoh N, Twiefel J. On developing an optimal design procedure for a bimorph piezoelectric cantilever energy harvester under a predefined volume. Mechanical Systems and Signal Processing. 2018;106:1-12. 
[32] Zeng S, Zhang C, Wang K, Wang B, Sun L. Analysis of delamination of unimorph cantilever piezoelectric energy harvesters. Journal of Intelligent Material Systems and Structures. 2018;29(9):1875-83.

[33] Thein CK, Ooi BL, Liu J-S, Gilbert JM. Modelling and optimisation of a bimorph piezoelectric cantilever beam in an energy harvesting application. J Eng Sci Technol. 2016;11(2):212-27.

[34] Lee B, Lin S, Wu W, Wang X, Chang P, Lee C. Piezoelectric MEMS generators fabricated with an aerosol deposition PZT thin film. Journal of Micromechanics and Microengineering. 2009;19(6):065014.

[35] Kim S-B, Park H, Kim S-H, Wikle HC, Park J-H, Kim D-J. Comparison of MEMS PZT cantilevers based on $e_{-}\{31\}$ and $e_{-}\{33\}$ modes for vibration energy harvesting. Journal of microelectromechanical systems. 2013;22(1):26-33.

[36] Kazmierski TJ, Beeby S. Energy harvesting systems: Springer, 2014.

[37] Kim C-K. Theoretical comparison of the energy conversion efficiencies of electrostatic energy harvesters. Journal of the Korean Physical Society. 2017;70(3):2929.

[38] Mahmoud M, Abdel-Rahman EM, Mansour R, El-Saadany E. Out-of-Plane Continuous Electrostatic Micro-Power Generators. Sensors. 2017;17(4):877.

[39] Lee C, Lim YM, Yang B, Kotlanka RK, Heng C-H, He JH, et al. Theoretical comparison of the energy harvesting capability among various electrostatic mechanis ms from structure aspect. Sensors and Actuators A: Physical. 2009;156(1):208-16.

[40] Mur Miranda JO. Electrostatic vibration-to-electric energy conversion: Massachusetts Institute of Technology, 2004.

[41] Tao K, Lye SW, Miao J, Hu X. Design and implementation of an out-of-plane electrostatic vibration energy harvester with dual-charged electret plates. Microelectronic Engineering. 2015;135:32-7.

[42] Ahmed S, Kakkar V. An Electret-Based Angular Electrostatic Energy Harvester for Battery-Less Cardiac and Neural Implants. IEEE Access. 2017;5:19631-43. 
[43] Suzuki M, Shimokizaki M, Takahashi T, Yoshikawa Y, Aoyagi S. Fabrication and Characterization of Nano/Micro Textured Electret to Avoid Electrostatic Stiction and Enhance Its Surface Potential. Journal of Physics: Conference Series. 2015;660(1):012042.

[44] Fujita T, Toyonaga T, Nakade K, Kanda K, Higuchi K, Maenaka K. Selective electret charging method for energy harvesters using biased electrode. Procedia Engineering. 2010;5:774-7.

[45] Onishi T, Fujita T, Fujii K, Kanda K, Maenaka K, Higuchi K. Selective electret charging method of $\mathrm{SiO}<\mathrm{inf}>2</ \mathrm{inf}\rangle$ film for energy harvesters by using biased electrode. Conference Selective electret charging method of $\mathrm{SiO}<\mathrm{inf}>2</ \mathrm{inf}>$ film for energy harvesters by using biased electrode. p. 1-5.

[46] Jeon YB, Sood R, Jeong Jh, Kim SG. MEMS power generator with transverse mode thin film PZT. Sensors and Actuators A: Physical. 2005;122(1):16-22.

[47] Choi WJ, Jeon Y, Jeong J-H, Sood R, Kim SG. Energy harvesting MEMS device based on thin film piezoelectric cantilevers. Journal of Electroceramics. 2006;17(2):5438.

[48] Basrour S, Charlot B, Marzencki M, Grasso A, Colin M, Valbin L. Design and fabrication of piezoelectric micro power generators for autonomous microsystems. Conference Design and fabrication of piezoelectric micro power generators for autonomous microsystems, Montreux, Switzerland. TIMA, p. 299-302.

[49] Marzencki M, Ammar Y, Basrour S. Integrated power harvesting system including a MEMS generator and a power management circuit. Sensors and Actuators A: Physical. 2008;145-146:363-70.

[50] Muralt P, Marzencki M, Belgacem B, Calame F, Basrour S. Vibration Energy Harvesting with PZT Micro Device. Procedia Chemistry. 2009;1(1):1191-4.

[51] Elfrink R, Kamel TM, Goedbloed M, Matova S, Hohlfeld D, Andel Yv, et al. Vibration energy harvesting with aluminum nitride-based piezoelectric devices. Journal of Micromechanics and Microengineering. 2009;19(9):094005. 
[52] Fang H-B, Liu J-Q, Xu Z-Y, Dong L, Chen D, Cai B-C, et al. A MEMS-based piezoelectric power generator for low frequency vibration energy harvesting. Chinese Physics Letters. 2006;23(3):732-4.

[53] Renaud M, Karakaya K, Sterken T, Fiorini P, Van Hoof C, Puers R. Fabrication, modelling and characterization of MEMS piezoelectric vibration harvesters. Sensors and Actuators A: Physical. 2008;145(Supplement C):380-6.

[54] Dongna S, Jung-Hyun P, Jyoti A, Song-Yul C, Howard CW, III, Dong-Joo K. The design, fabrication and evaluation of a MEMS PZT cantilever with an integrated Si proof mass for vibration energy harvesting. Journal of Micromechanics and Microengineering. 2008;18(5):055017.

[55] Gu L, Livermore C. Impact-driven, frequency up-converting coupled vibration energy harvesting device for low frequency operation. Smart Materials and Structures. 2011;20(4):045004.

[56] Meninger S, Mur-Miranda JO, Amirtharajah R, Chandrakasan A, Lang JH. Vibration-to-electric energy conversion. IEEE Transactions on Very Large Scale Integration (VLSI) Systems. 2001;9(1):64-76.

[57] Ma W, Wong M, Ruber L. Dynamic simulation of an implemented electrostatic power micro-generator. Conference Dynamic simulation of an implemented electrostatic power micro-generator. p. 380-5.

[58] Kuehne I, Frey A, Marinkovic D, Eckstein G, Seidel H. Power MEMS - A capacitive vibration-to-electrical energy converter with built-in voltage. Sensors and Actuators A: Physical. 2008;142(1):263-9.

[59] Chiu Y, Tseng VF. A capacitive vibration-to-electricity energy converter with integrated mechanical switches. Journal of Micromechanics and Microengineering. 2008;18(10):104004.

[60] El-hami M, Glynne-Jones P, White NM, Hill M, Beeby S, James E, et al. Design and fabrication of a new vibration-based electromechanical power generator. Sensors and Actuators A: Physical. 2001;92(1):335-42. 
[61] Sari I, Balkan T, Kulah H. An electromagnetic micro power generator for wideband environmental vibrations. Sensors and Actuators A: Physical. 2008;145-146:405-13.

[62] Cho HJ, Ahn CH. A bidirectional magnetic microactuator using electroplated permanent magnet arrays. Journal of Microelectromechanical Systems. 2002;11(1):7884.

[63] Han M, Yuan Q, Sun X, Zhang H. Design and Fabrication of Integrated Magnetic MEMS Energy Harvester for Low Frequency Applications. Journal of Microelectromechanical Systems. 2014;23(1):204-12.

[64] Mitcheson PD, Yeatman EM, Rao GK, Holmes AS, Green TC. Energy harvesting from human and machine motion for wireless electronic devices. Proceedings of the IEEE. 2008;96(9):1457-86.

[65] Lee BS, Wu W-J, Shih WP, Vasic D, Costa F. Power Harvesting using Piezoelectric MEMS Generator with Interdigital Electrodes. Conference Power Harvesting using Piezoelectric MEMS Generator with Interdigital Electrodes, New-York, United States. p.

[66] Lee BS, Lin SC, Wu WJ, Wang XY, Chang PZ, Lee CK. Piezoelectric MEMS generators fabricated with an aerosol deposition PZT thin film. Journal of Micromechanics and Microengineering. 2009;19(6):065014.

[67] Youngsman JM, Luedeman T, Morris DJ, Anderson MJ, Bahr DF. A model for an extensional mode resonator used as a frequency-adjustable vibration energy harvester. Journal of Sound and Vibration. 2010;329(3):277-88.

[68] Xue H, Hu Y, Wang Q-M. Broadband piezoelectric energy harvesting devices using multiple bimorphs with different operating frequencies. IEEE transactions on ultrasonics, ferroelectrics, and frequency control. 2008;55(9).

[69] Erturk A, Hoffmann J, Inman D. A piezomagnetoelastic structure for broadband vibration energy harvesting. Applied Physics Letters. 2009;94(25):254102.

[70] Ghandchi Tehrani M, Elliott SJ. Extending the dynamic range of an energy harvester using nonlinear damping. Journal of Sound and Vibration. 2014;333(3):623-9. 
[71] Mann BP, Sims ND. Energy harvesting from the nonlinear oscillations of magnetic levitation. Journal of Sound and Vibration. 2009;319(1):515-30.

[72] Roundy S, Zhang Y. Toward self-tuning adaptive vibration-based microgenerators. Conference Toward self-tuning adaptive vibration-based microgenerators, vol. 5649. SPIE, p. 12.

[73] Eli SL, Paul KW. Resonance tuning of piezoelectric vibration energy scavenging generators using compressive axial preload. Smart Materials and Structures. 2006;15(5):1413.

[74] Yuantai H, Huan X, Hongping H. A piezoelectric power harvester with adjustable frequency through axial preloads. Smart Materials and Structures. 2007;16(5):1961.

[75] Marzencki M, Defosseux M, Basrour S. MEMS Vibration Energy Harvesting Devices With Passive Resonance Frequency Adaptation Capability. Journal of Microelectromechanical Systems. 2009;18(6):1444-53.

[76] Zhu D, Roberts S, Tudor MJ, Beeby SP. Design and experimental characterization of a tunable vibration-based electromagnetic micro-generator. Sensors and Actuators A: Physical. 2010;158(2):284-93.

[77] Vinod RC, Prasad MG, Frank TF. Towards an autonomous self-tuning vibration energy harvesting device for wireless sensor network applications. Smart Materials and Structures. 2011;20(2):025004.

[78] Miller LM, Pillatsch P, Halvorsen E, Wright PK, Yeatman EM, Holmes AS. Experimental passive self-tuning behavior of a beam resonator with sliding proof mass. Journal of Sound and Vibration. 2013;332(26):7142-52.

[79] Zhang Y, Wang T, Luo A, Hu Y, Li X, Wang F. Micro electrostatic energy harvester with both broad bandwidth and high normalized power density. Applied Energy. 2018;212:362-71.

[80] Triplett A, Quinn DD. The Effect of Non-linear Piezoelectric Coupling on Vibrationbased Energy Harvesting. Journal of Intelligent Material Systems and Structures. 2009;20(16):1959-67. 
[81] Elshurafa AM, Khirallah K, Tawfik HH, Emira A, Aziz AKSA, Sedky SM. Nonlinear Dynamics of Spring Softening and Hardening in Folded-MEMS Comb Drive Resonators. Journal of Microelectromechanical Systems. 2011;20(4):943-58.

[82] Vocca H, Neri I, Travasso F, Gammaitoni L. Kinetic energy harvesting with bistable oscillators. Applied Energy. 2012;97:771-6.

[83] Zhou S, Cao J, Inman DJ, Lin J, Liu S, Wang Z. Broadband tristable energy harvester: Modeling and experiment verification. Applied Energy. 2014;133:33-9.

[84] Shi S, Xuhan D, Yunna S, Xiaojian X, Guifu D, Xiaolin Z. MEMS-based widebandwidth electromagnetic energy harvester with electroplated nickel structure. Journal of Micromechanics and Microengineering. 2017;27(11):115007.

[85] Leadenham S, Erturk A. M-shaped asymmetric nonlinear oscillator for broadband vibration energy harvesting: Harmonic balance analysis and experimental validation. Journal of Sound and Vibration. 2014;333(23):6209-23.

[86] Gafforelli G, Corigliano A, Xu R, Kim S-G. Experimental verification of a bridgeshaped, nonlinear vibration energy harvester. Applied Physics Letters. 2014;105(20):203901.

[87] Hajati A, Kim S-G. Ultra-wide bandwidth piezoelectric energy harvesting. Applied Physics Letters. 2011;99(8):083105.

[88] Mallick D, Amann A, Roy S. High Figure of Merit Nonlinear Microelectromagne tic Energy Harvesters for Wideband Applications. Journal of Microelectromechanical Systems. 2017;26(1):273-82.

[89] Liu H, How Koh K, Lee C. Ultra-wide frequency broadening mechanism for microscale electromagnetic energy harvester. Applied Physics Letters. 2014;104(5):053901.

[90] Erturk A, Inman DJ. Piezoelectric energy harvesting: John Wiley \& Sons, 2011.

[91] Ajitsaria J, Choe S-Y, Shen D, Kim D. Modeling and analysis of a bimorph piezoelectric cantilever beam for voltage generation. Smart Materials and Structures. 2007;16(2):447. 
[92] Lin J, Wu X, Ren T, Liu L. Modeling and simulation of piezoelectric MEMS energy harvesting device. Integrated Ferroelectrics. 2007;95(1):128-41.

[93] Madinei H, Rezazadeh G, Azizi S. Stability and Bifurcation Analysis of an Asymmetrically Electrostatically Actuated Microbeam. Journal of Computational and Nonlinear Dynamics. 2015;10(2):021002--8.

[94] Mitcheson PD, Miao P, Stark BH, Yeatman EM, Holmes AS, Green TC. MEMS electrostatic micropower generator for low frequency operation. Sensors and Actuators A: Physical. 2004;115(2):523-9.

[95] Nizar J, Abdallah R, Armando AAC, Mohammad IY. Higher order modes excitation of electrostatically actuated clamped-clamped microbeams: experimental and analytical investigation. Journal of Micromechanics and Microengineering. 2016;26(2):025008.

[96] Fang H-B, Liu J-Q, Xu Z-Y, Dong L, Wang L, Chen D, et al. Fabrication and performance of MEMS-based piezoelectric power generator for vibration energy harvesting. Microelectronics Journal. 2006;37(11):1280-4.

[97] Khodaparast HH. Stochastic finite element model updating and its application in aeroelasticity: University of Liverpool, 2010.

[98] Sandberg D, Mansour R, Olsson M. Fatigue probability assessment including aleatory and epistemic uncertainty with application to gas turbine compressor blades. International Journal of Fatigue. 2017;95:132-42.

[99] Vanmarcke E. Random fields: analysis and synthes is: World scientific, 2010.

[100] Koehler JR, Owen AB. 9 Computer experiments. Handbook of Statistics: Elsevier; 1996. p. 261-308.

[101] Lin R, Wang W. Structural dynamics of microsystems - current state of research and future directions. Mechanical systems and signal processing. 2006;20(5):1015-43.

[102] Alexeenko A, Chigullapalli S, Zeng J, Guo X, Kovacs A, Peroulis D. Uncertainty in microscale gas damping: Implications on dynamics of capacitive MEMS switches. Reliability Engineering \& System Safety. 2011;96(9):1171-83. 
[103] Gurav S, Kasyap A, Sheplak M, Cattafesta L, Haftka R, Goosen J, et al. Uncertainty-Based Design Optimization of a Micro Piezoelectric Composite Energy Reclamation Device. 10th AIAA/ISSMO Multidisciplinary Analysis and Optimization Conference: American Institute of Aeronautics and Astronautics; 2004.

[104] Shanmugavalli M, Uma G, Vasuki B, Umapathy M. Design and Simulation of MEMS Devices using Interval Analysis. Journal of Physics: Conference Series. 2006;34(1):601.

[105] Agarwal N, Aluru NR. Stochastic modeling of coupled electromechanical interaction for uncertainty quantification in electrostatically actuated MEMS. Computer Methods in Applied Mechanics and Engineering. 2008;197(43):3456-71.

[106] Agarwal N, Aluru NR. A data-driven stochastic collocation approach for uncertainty quantification in MEMS. International Journal for Numerical Methods in Engineering. 2010;83(5):575-97.

[107] Younis MI. MEMS Linear and Nonlinear Statics and Dynamics. 2011.

[108] Madinei H, Khodaparast HH, Adhikari S, Friswell MI. Design of MEMS piezoelectric harvesters with electrostatically adjustable resonance frequency. Mechanical Systems and Signal Processing. 2016;81:360-74.

[109] Abdelkefi A, Barsallo N. Comparative modeling of low-frequency piezomagnetoelastic energy harvesters. Journal of Intelligent Material Systems and Structures. 2014;25(14):1771-85.

[110] Abdelmoula H, Zimmerman S, Abdelkefi A. Accurate modeling, comparative analysis, and performance enhancement of broadband piezoelectric energy harvesters with single and dual magnetic forces. International Journal of Non-Linear Mechanics. 2017;95:355-63. 